Aus der Abteilung Psychiatrie und Psychotherapie

(Prof. Dr. med. P. Falkai)

im Zentrum Psychosoziale Medizin

der Medizinischen Fakultät der Universität Göttingen

\title{
Veränderungen kardiovaskulärer Risikofaktoren \\ - mit besonderer Betrachtung von Homocystein und hsCRP - unter drei aktuell diskutierten Ernährungsstrategien zur Gewichtsreduktion:
}

Low carb, Low fat und Low fat with reduced glycemic load

\author{
Inaugural-Dissertation \\ zur Erlangung des Doktorgrades \\ der Medizinischen Fakultät \\ der Georg-August-Universität zu Göttingen
}

vorgelegt von

Ines Hönemann

aus Vechta 
Dekan:

I. Berichterstatter: Prof. Dr. rer. nat. V. Pudel

II. Berichterstatter/in:.

III. Berichterstatter/in:

Tag der mündlichen Prüfung:
Prof. Dr. med. P. Falkai 
1 Einleitung ...................................................................................................................... 5

1.1 ERNÄHRUNG IN THERAPIE UND PRÄVENTION VON ADIPOSITAS UND KARDIOVASKULÄREN

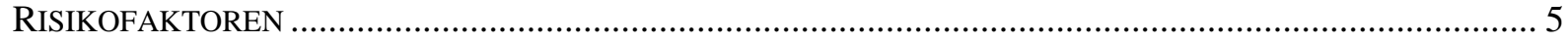

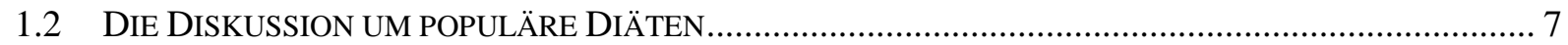

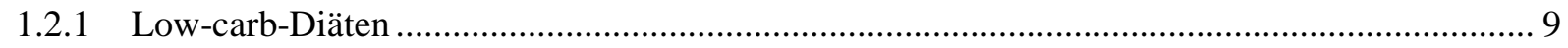

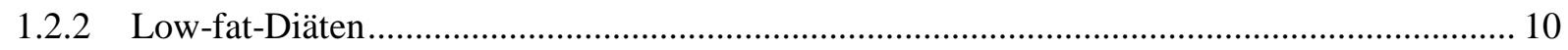

1.2.3 Das Konzept des glykämischen Index und der glykämischen Last .......................................... 11

1.3 KARDIOVASKULÄRE RISIKOFAKTOREN ALS GÜTEKRITERIEN EINER DIÄT ................................ 13

1.3.1 C-reaktives Protein und hoch sensitives C-reaktives Protein................................................. 13

1.3.2 Homocystein - kardiovaskulärer Risikofaktor, Marker des Vitaminstatus oder beides? .......... 15

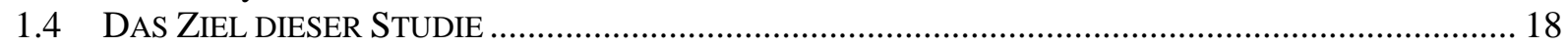

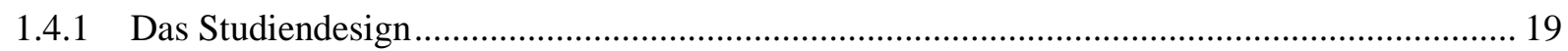

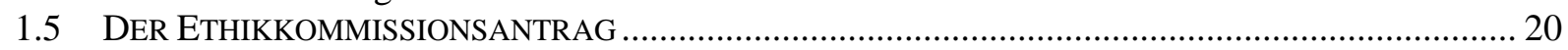

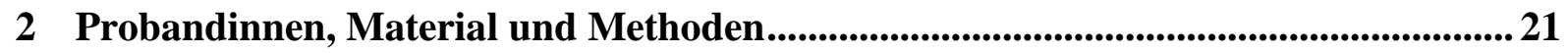

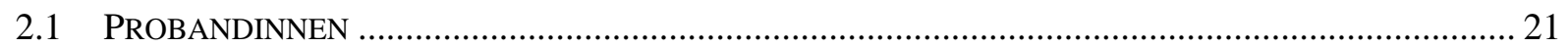

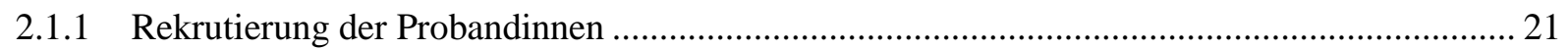

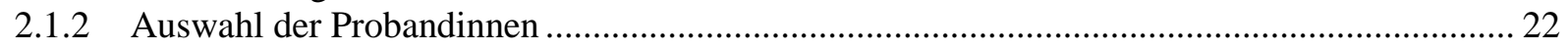

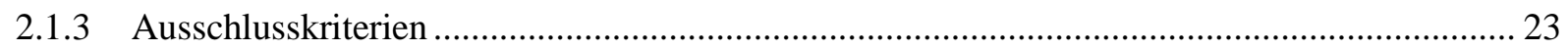

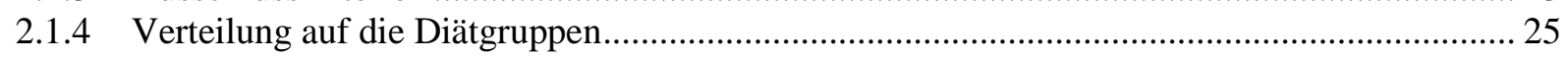

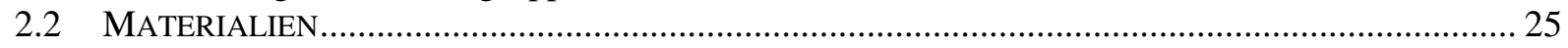

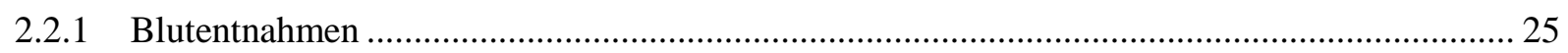

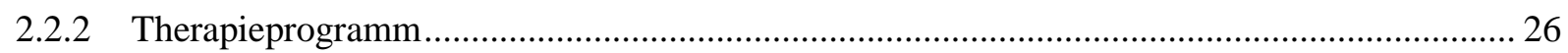

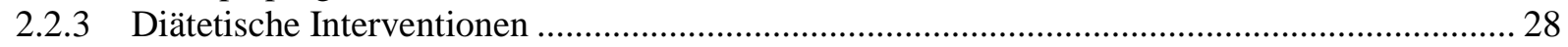

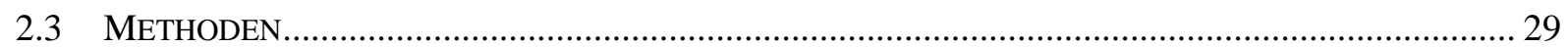

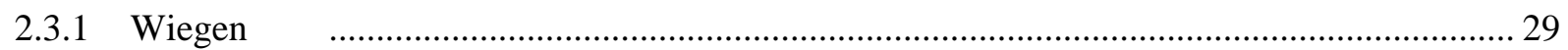

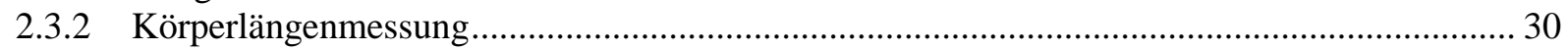

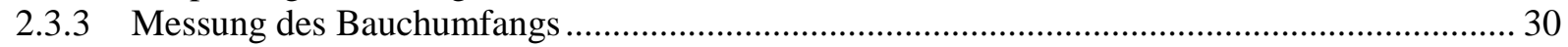

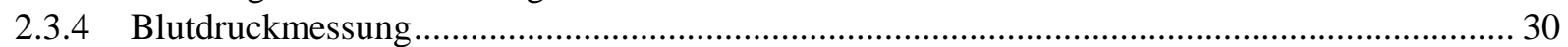

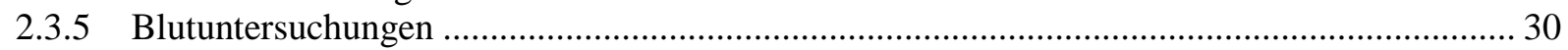

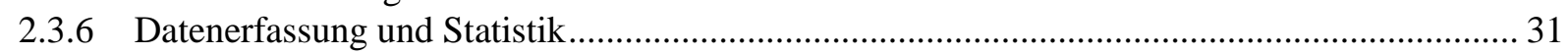

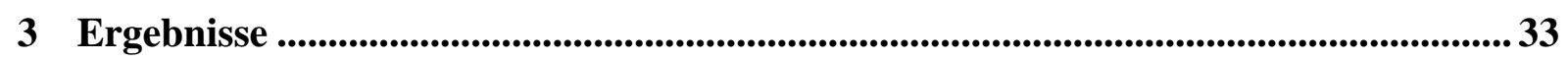

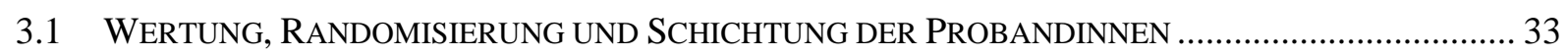

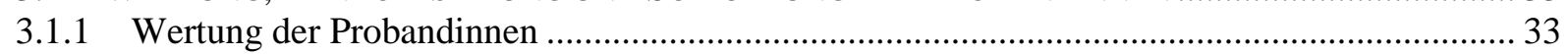

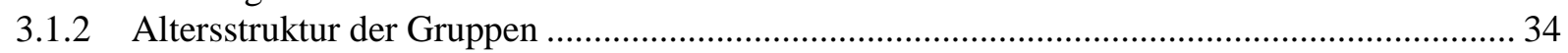

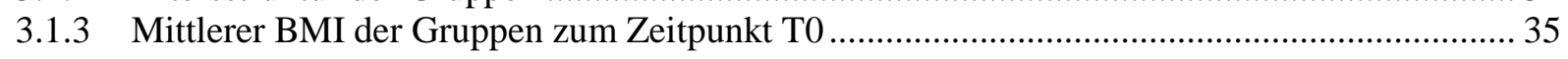

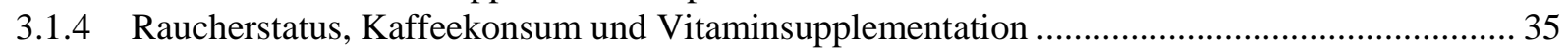

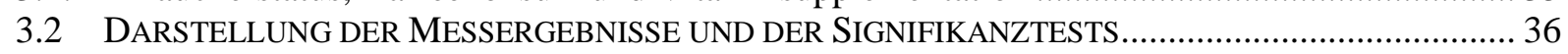

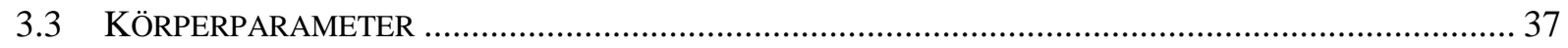

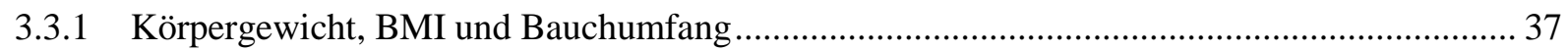

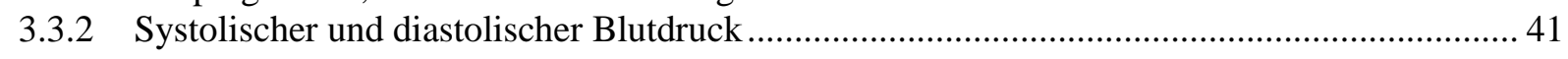

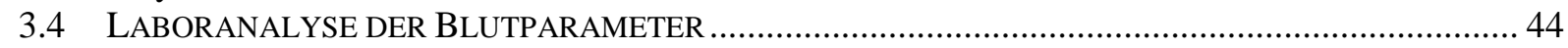

3.4.1 Homocystein, MTHFR und hoch sensitives C-reaktives Protein.............................................. 44

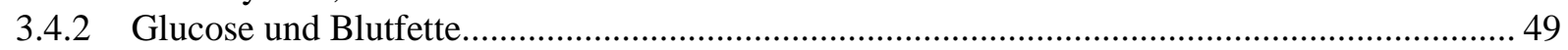

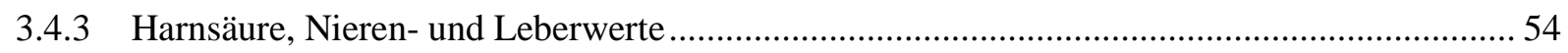

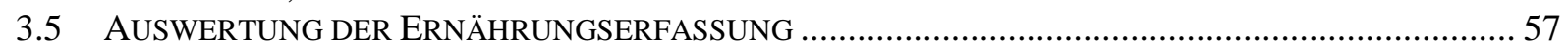

3.5.1 Kalorien, Makronährstoffe und ausgewählte Mikronährstoffe ................................................... 57

3.5.2 Analyse der Compliance anhand des Makronährstoffverzehrs ............................................... 67

3.6 KORRELATIONSANALYSEN FÜR DIE KÖRPERMESSWERTE, LABOR- UND VERZEHRDATEN ........... 67

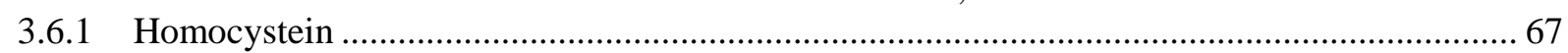

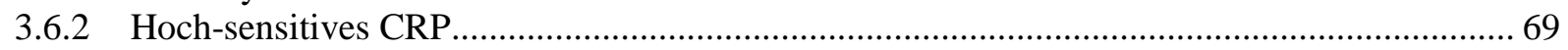




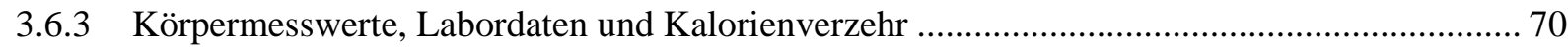

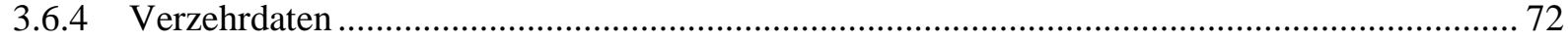

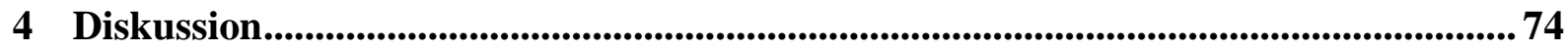

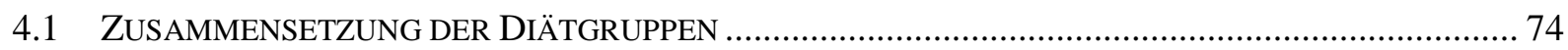

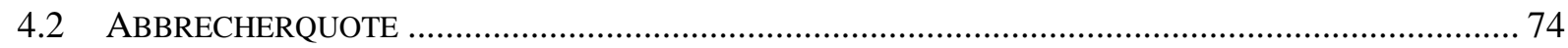

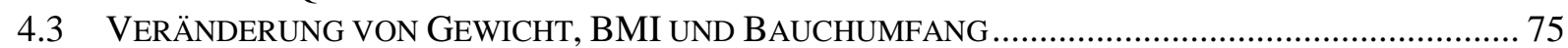

4.4 AUSWIRKUNGEN AUF DEN SYSTOLISCHEN UND DIASTOLISCHEN BLUTDRUCK ............................ 76

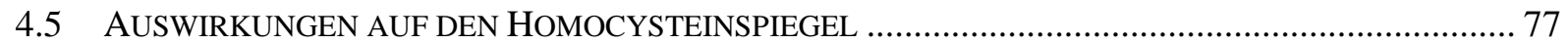

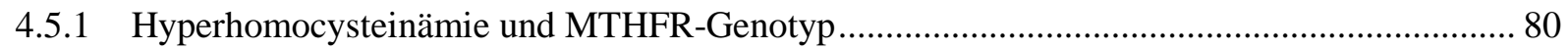

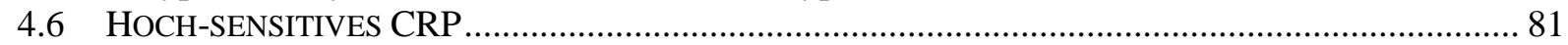

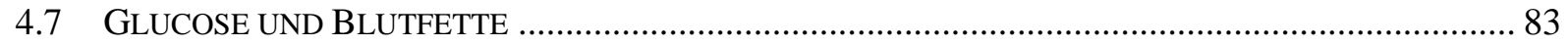

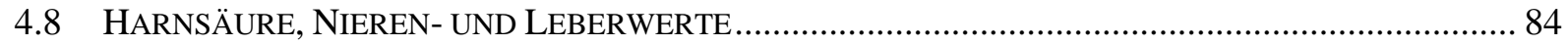

4.9 VERZEHRDATEN VON KALORIEN, MAKRONÄHRSTOFFEN UND AUSGEWÄHLTEN

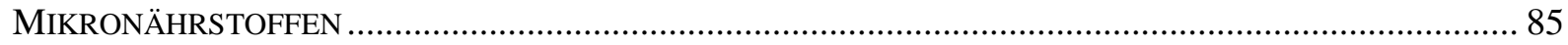

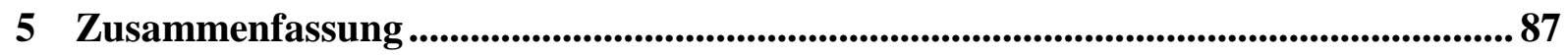

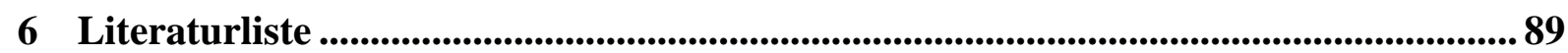




\section{Einleitung}

Übergewicht und Adipositas sind heute die häufigsten chronischen Gesundheitsstörungen in den Industrienationen. Nach der derzeit gültigen Einteilung von Übergewicht oder Präadipositas und Adipositas anhand des Body-Mass-Index hat der mittlere BMI der erwachsenen Bevölkerung in Europa mit 26,5 kg/m² bereits den Gewichtsbereich des Übergewichts erreicht (WHO 2000, WHO 2005). Besonders die Vermehrung des viszeralen Fettgewebes wirkt sich negativ auf andere Erkrankungen wie Diabetes mellitus Typ 2, Hyperlipoproteinämie, Hypertonus, Atherosklerose, Hyperurikämie bis hin zu Störungen der Blutgerinnung, degenerativen Gelenkerkrankungen und diversen Krebserkrankungen aus. Die daraus resultierende erhöhte Morbidität und Mortalität erweist sich nicht nur für die betroffenen Menschen als außerordentliche Bürde. Sie hat darüber hinaus immense negative ökonomische Folgen für Gesundheitssysteme und Volkswirtschaften.

Dagegen kommt es durch Verminderung der Adipositas beim einzelnen Menschen gleichzeitig zur Verbesserung von Begleiterkrankungen und von möglicherweise bereits bestehenden Folgeerkrankungen. Die Morbidität und Mortalität der Bevölkerung sinkt. Daher sind therapeutische Wege zum Bekämpfen der Adipositas medizinisch notwendig und ökonomisch wünschenswert. Entsprechend hält das Forschungsinteresse am Themenkomplex Adipositas und Adipositastherapie unvermindert an.

\subsection{Ernährung in Therapie und Prävention von Adipositas und kardiovaskulären Risikofaktoren}

Es ist heute aus vielen Untersuchungen bekannt, dass Übergewicht und Adipositas und die oft mit der Vermehrung des Fettgewebes einhergehenden Krankheiten wie Diabetes mellitus Typ 2, Hyperlipoproteinämie und Bluthochdruck Risikofaktoren für Atherosklerose und kardiovaskuläre Erkrankungen darstellen. Allen diesen Krankheiten ist darüber hinaus gemeinsam, dass sie mit einem sportlich-aktiven Lebensstil und bestimmten Ernährungsstrategien positiv beeinflusst werden können. $\mathrm{Zu}$ einem gesunden und präventiv wirksamen Lebensstil gehören regelmäßige Bewegung, Nikotinverzicht, ein höchstens maßvoller Alkoholgenuss und eine vollwertige, gesunde Ernährung. Letztere besteht laut Empfehlung der DGE aus reichlich Kartoffeln und Getreideprodukten (möglichst aus Vollkorngetreide), 5 Portionen Obst und Gemüse pro Tag, täglich Milch oder Milchprodukten, ein- bis zweimal wöchentlich Fisch und aus insgesamt wenig Fleisch, Fett, Eier, Salz und Zucker. Damit kann sowohl den 
genannten Risikofaktoren kardiovaskulärer Erkrankungen als auch einer Adipositas vorgebeugt beziehungsweise diese therapiert werden.

Zur sinnvollen und erfolgreichen Therapie von Adipositas und kardiovaskulären Risikofaktoren sollten die Elemente eines gesunden Lebensstils in ein therapeutisches Gesamtkonzept integriert werden. Die mit dem Thema beschäftigten deutschen Fachgesellschaften empfehlen in ihren gemeinsamen evidenzbasierten Leitlinien zur Adipositastherapie folgendes: Gesunde Ernährung, Bewegung/Sport, Alkohol- und Nikotinverzicht, eine medizinische Betreuung und gegebenenfalls eine verhaltenstherapeutische Behandlung (Hauner et al. 2005). Insbesondere für Menschen mit einer Adipositas höheren Grades dürften die letzten beiden Punkte nicht zu vernachlässigen sein.

In der Realität finden die meisten Versuche von Gewichtsreduktionen allerdings in der häuslichen Umgebung und ohne professionelle Hilfe statt. Viele übergewichtige und adipöse Menschen wollen ihr Gewicht reduzieren. Ihre Motivation ist häufig ihr körperliches Erscheinungsbild, welches nicht dem gesellschaftlichen Idealbild des schlanken Menschen entspricht, nicht ihr Wohlbefinden oder medizinische Gründe (Freedman et al. 2001). Die große Anzahl von Zeitschriften und Büchern mit Diätvorschlägen zum „Abnehmen“ bietet diesen Menschen scheinbar Hilfe an. Die Ernährung wird in den entsprechenden Medien nicht selten als einziger Therapiebaustein zur Gewichtsreduzierung thematisiert. Besonders Diätvarianten mit unterschiedlicher Makronährstoffzusammensetzung werden in den letzten Jahren stark angepriesen: Durch kohlenhydratarme oder fettarme Ernährungsstrategien oder eine Ernährung mit Reduktion des glykämischen Index, so genannte Glyx-Diäten, suggeriert die Werbung einen schnellen Erfolg bei der Gewichtsabnahme als Marketingstrategie für einen aktuell gewinnträchtigen Markt.

Ernährung hat jedoch weitaus mehr Funktionen als nur die Erhaltung oder Erzielung eines Wunschkörpergewichts zu gewährleisten. Durch die Nahrung müssen dem Organismus alle wichtigen Bausteine für seine Gesunderhaltung in erforderlichem Maß zugeführt werden. In geringen Mengen benötigte Nährstoffe wie Vitamine oder Spurenelemente werden als Bestandteile einer vollwertigen Nahrung ausreichend aufgenommen. Durch eine Reduktionsdiät oder Ernährung mit unausgewogener Makronährstoffzusammensetzung kann hingegen ein klinisch relevantes Missverhältnis zwischen Zufuhr und Bedarf eines Nährstoffs auftreten. Der Mangel, aber auch eine Überdosierung von bestimmten Nährstoffen, kann Gesundheitsstörungen hervorrufen. So kann die erhöhte Cholesterinzufuhr zu einer Hypercholesterinämie mit ihren negativen Folgen für die Gesundheit führen. Eisenmangelanämie, Rachitis (Kno- 
chenerkrankung durch Vitamin-D-Mangel) und Skorbut (Erkrankung durch Vitamin-CMangel) sind andererseits einige der bekannteren Erkrankungen, die durch Mikronährstoffmangel hervorgerufen werden. Ein weiteres Beispiel aus der klinischen Medizin ist die Hyperhomocysteinämie, die als einer der neuen kardiovaskulären Risikofaktoren gilt. Sie wird ebenfalls oft durch einen Vitaminmangel ausgelöst: einen Mangel an Folsäure, Vitamin B6 oder/ und B12. Das Risiko für das Auftreten von Herz-Kreislauf-Erkrankungen liegt jedoch auch unabhängig vom Bestehen einer Hyperhomocysteinämie bei geringer täglicher Folsäureaufnahme signifikant höher als bei hoher Zufuhr dieses Vitamins (Holmquist et al. 2003). Ein Folsäuremangel übt einen negativen Einfluss auf kardiovaskuläre Erkrankungen aus. Dies ergibt auch eine Untersuchung von Verhaar et al. (Verhaar et al. 2002).

Weitere Vitaminmangelzustände wie ein Mangel von Vitamin E, C und B-Carotin stehen in negativem Zusammenhang zu entzündlichen Reaktionen. Die Oxidation freier Radikale und die Regeneration der körpereigenen Abwehrsysteme werden durch den Mangel dieser Vitamine beeinträchtigt und Entzündungsvorgänge bleiben dadurch ungebremst. Aber auch andere Nahrungsinhaltsstoffe wie Ballaststoffe unterstützen die Gesunderhaltung des Körpers. Bei ausreichender Ballaststoffzufuhr liegen die Blutwerte für bestimmte Entzündungsparameter niedriger (King et al. 2007). Die genannten Beispiele zeigen die vielfältige Wirkung ganz unterschiedlicher Nahrungsbestandteile. Und bis heute sind noch lange nicht alle Funktionen der einzelnen Nährstoffe auf den Organismus entschlüsselt worden.

\subsection{Die Diskussion um populäre Diäten}

Das zunehmende Wissen um die Bedeutung von Ernährung für die Gesundheit wie auch für die Entstehung beziehungsweise Verschlechterung von krankhaften Zuständen hat eine Debatte unter Ernährungsexperten entfacht. Noch vor einigen Jahren wurde eine hypokalorische Kost zum Erreichen einer negativen Energiebalance als oberste Maxime und ausschlaggebend für eine Gewichtsreduktion angesehen. Heute misst man dagegen den einzelnen Nahrungskomponenten einen größeren Wert bei. Verschiedene Theorien und Hypothesen zur Entstehung der Adipositas speisen sich aus Untersuchungen und epidemiologischen Daten. Sie schreiben entweder Fett oder Kohlenhydraten oder Kohlenhydraten mit hohem glykämischem Index die Hauptschuld am Übergewicht der Bevölkerung zu. Neue Erkenntnisse über die Wirkung unterschiedlicher Nährstoffe unterstützen diese Überlegungen. Das hat dazu geführt, dass sogar direkt konträre Ernährungsstrategien für Therapie und Prävention von Übergewicht erprobt wurden und werden, beispielsweise kohlenhydratarme oder fettarme Diäten. 
Nicht nur die geringen Erfolge bisheriger Ernährungstherapien zur Gewichtsreduktion, sondern auch ihre umstrittene gesundheitliche Güte treiben die medizinische Diskussion und Bewertung von Diäten weiter an. Verschiedene populäre Diätstrategien, die die Empfehlungen der Fachgesellschaften teilweise auf den Kopf stellen, sind ein besonderer Streitpunkt wie etwa die Ernährungsempfehlungen des Arztes Robert Atkins. Er publizierte seine „DiätRevolution“ - wie er das Konzept selbst nannte - erstmals 1972, in deutscher Übersetzung 1974 (Atkins und Herwood 1974) und erneut 1998 (Atkins 1998).

In der medizinischen Fachliteratur finden sich seit dieser Publikation und verstärkt seit Anfang der 1990er Jahre zahlreiche Artikel über medizinische Untersuchungen zum Thema Diäten oder Ernährungsstrategien zur Gewichtsreduktion. Studien zu Nutzen und Risiken verschiedener Diätstrategien sollen im Zeitalter der evidenzbasierten Medizin Fakten über ihre Effektivität in Bezug auf eine Gewichtsreduktion und über gesundheitliche Vor- und Nachteile der jeweiligen Kostform liefern. Je nach Fragestellung werden zur Überprüfung der Wirksamkeit einer Ernährungsweise verschiedene klinische Messwerte und Laborparameter erhoben, um die Auswirkungen der jeweiligen Ernährung über einen bestimmten Zeitraum und gegenüber anderen Ernährungsstrategien zu testen. Bisher finden sich unter verschiedensten Diätregimen nur bezüglich der meist mäßigen Gewichtsreduktion und der dennoch gleichzeitig erzielten Verbesserung in Bezug auf die adipositas-assoziierten kardiovaskulären Risikofaktoren ähnliche Ergebnisse (Dansinger et al. 2005). Für die beginnende Verbesserung kardiovaskulärer Risikofaktoren braucht der Nettogewichtsverlust pro Person interessanterweise nur wenige Prozentpunkte des initialen Gewichts betragen (Anderson und Konz 2001).

Da in den meisten Studien über eine relativ kurze Zeit von wenigen Wochen bis maximal 1-2 Jahren das Outcome der Patienten beobachtet wurde, ist über einen länger anhaltenden Effekt all dieser Ernährungsstrategien bisher wenig bekannt. Insbesondere für diätetische Behandlungen mit unausgewogenen Nährstoffrelationen werden gesundheitliche Bedenken von Kritikern angemeldet. Wenn ein Mikro- und/ oder Makronährstoffmangel diätetisch möglich erscheint oder der erhöhte Verzehr einzelner Nahrungsinhaltsstoffe bedenklich ist, muss mit negativen Auswirkungen auf die Gesundheit gerechnet oder können diese zumindest nicht ausgeschlossen werden (Meckling et al. 2004). Aus medizinischer Sicht sollte jedoch die gesundheitliche Unbedenklichkeit einer Gewichtsreduktionsdiät auf Dauer gewährleistet sein.

Entsprechend ihrer Popularität und Verbreitung in den Medien werden heute zur Gewichtsreduktion hauptsächlich Ernährungsstrategien mit geringem Kohlenhydratgehalt (low carbohydrates oder kurz low carb), geringem Fettgehalt (low fat) oder reduziertem glykä- 
misch wirksamen Gehalt der Nahrung (reduced glycemic load) untersucht. Gemeinsam ist diesen populären Diäten, dass sie jeweils einen Makronährstoff in der Nahrung beschränken. Entweder dürfen Kohlenhydrate beziehungsweise Kohlenhydrate mit hohem glykämisch wirksamen Potential oder Fette in beschränktem Maß verzehrt werden. Die jeweils anderen Makronährstoffe können in beliebiger Menge, also ad libitum, zugeführt werden. Dieses Prinzip vereinfacht eine diätetische Strategie gegenüber dem allgemeinen „Kalorienzählen“, welches sehr restriktiv und über einen längeren Zeitraum schwer durchzuhalten ist (Ellrott et al. 1998). Mit einer Ad-libitum-Strategie, also dem unbeschränkten Genuss einiger Lebensmittel, wird zusätzlich dem psychologischen Faktor Angst, während einer Diät Hunger leiden zu müssen, Rechnung getragen. Der Autor des Buches „Dr. Atkins Diet Revolution“ stellt genau diesen Punkt recht populistisch und dramatisch in seinem Werk dar (Atkins und Herwood 1974). Eine Ad-libitum-Strategie wird heute aber ebenso bei fettreduzierten Diäten wie beispielsweise den „Weight watchers“ eingesetzt.

Im Folgenden sollen drei der aktuell diskutierten Ernährungsstrategien zur Gewichtsreduktion vorgestellt werden:

\subsubsection{Low-carb-Diäten}

Diätetische Strategien werden üblicherweise als Low-carb-Diäten bezeichnet, wenn sie einen Verzehr von maximal $100 \mathrm{~g}$ verwertbaren Kohlenhydraten pro Tag erlauben. Zumindest wird dies aktuell von vielen Autoren als Grenze angesetzt, denn eine gültige Definition fehlt international bisher (Ellrott und Pudel 2005). Bekannt geworden sind kohlenhydratarme Diäten durch den bereits erwähnten US-amerikanischen Arzt Dr. Robert C. Atkins. Sie basieren auf der Annahme, dass bestimmte Menschen eine Stoffwechselstörung mit Unverträglichkeit für Kohlenhydrate haben (Atkins und Herwood 1974). Die wissenschaftliche Erklärung geht heute davon aus, dass es durch den Konsum von einfachen Kohlenhydraten zu einer überschießenden Insulinausschüttung kommt, die den Blutzuckerspiegel übermäßig senken kann. Beim prädisponierten Menschen kommt es konsekutiv zu einer Hypoglykämie mit anschlieBendem Hungergefühl. Ein hoher Insulinspiegel hemmt die Lipolyse und fördert die Lipogenese und der Körper kann die Energiereserven aus den Fettspeichern nicht nutzen. Der Mensch isst trotz vorhandener Energiespeicher erneut. Es entsteht ein Circulus vitiosus.

Durch den anfänglichen Verzicht und die folgende Beschränkung der Kohlenhydrate in einer kohlenhydratarmen Diät soll die Insulinausschüttung vermindert werden. Ein niedriger Insulinspiegel vermindert das Hungergefühl und die Einlagerung der aufgenommenen Fett- 
säuren ins Fettgewebe. Gleichzeitig wird durch den niedrigen Insulinspiegel die Lipolyse gefördert und Fettgewebe abgebaut. Letzteres kann am Erscheinen von Ketonkörpern in Blut und Urin gemessen werden. Darüber hinaus führen die Ketonkörper vermutlich zu einem gedämpften Appetit, wodurch die Verminderung der Nahrungsaufnahme unterstützt wird. Diesen Mechanismen schreibt man eine tragende Rolle bei der Gewichtsabnahme unter Lowcarb-Diäten zu.

Die tatsächliche Effektivität von kohlenhydratarmen Diäten zur Gewichtsreduktion konnte in den letzten Jahren in mehreren wissenschaftlichen Untersuchungen und Metaanalysen belegt werden (Bravata et al. 2003). Allerdings hat sich auch gezeigt, dass Menschen durchschnittlich während einer kohlenhydratarmen Ernährungsweise deutlich weniger Kalorien verzehren als unter anderen zum Vergleich eingesetzten Diätregimen. Deshalb könnte die vermehrte Gewichtsreduktion bei kohlenhydratarmer Ernährung in den genannten Untersuchungen hierdurch ebenfalls erklärt werden. Interessanterweise zeigte sich jedoch, dass überwiegend nur die initiale Gewichtsabnahme mit Low-carb-Diäten gegenüber Low-fat- oder konventionellen Diäten signifikant besser war, wenn überhaupt (Meckling et al. 2004). Wurden die Studienteilnehmer über einen längeren Zeitraum von 12 statt 6 Monaten beobachtet, nahmen die Menschen unter kohlenhydratarmer Ernährung schneller und deutlicher wieder zu, so dass sich am Ende des verlängerten Beobachtungszeitraums kein signifikanter Gewichtsunterschied mehr ergab. Die genauen Gründe dafür sind nicht klar. Mögliche Antworten sind der anfänglich größere Körperwasserverlust unter kohlenhydratarmer Ernährung, der reversibel ist, oder eine schlechtere Langzeitcompliance durch die limitierte Lebensmittelauswahl (Foster et al. 2003, Stern et al. 2004).

\subsubsection{Low-fat-Diäten}

Die Idee, fettreduzierte Diäten zur Gewichtsreduktion einzusetzen und die Gesundheit damit positiv zu beeinflussen, basiert auf mehreren Überlegungen. Fett ist ein energiedichter Nährstoff mit 9 kcal/g gegenüber Kohlenhydraten und Eiweiß mit je 4 kcal/g. Und da Übergewicht entsteht, wenn die Energiezufuhr den Energieverbrauch übersteigt, scheint es einfacher, etwas Fett statt die doppelte Menge an Kohlenhydraten diätetisch einzusparen. In einem Review-Artikel stellten Bray und Popkin 1998 anhand der Analyse vieler Studien den Zusammenhang zwischen Nahrungsfettkonsum und höherem Gewicht sowie der Möglichkeit der Gewichtsreduktion unter fettreduzierter Ernährung dar (Bray und Popkin 1998). Selbst relativ einfach praktizierbare Konzepte wie der Ersatz fettreicher durch fettreduzierte Lebensmittel 
bei freiem Verzehr zeigen sich mit einer durchschnittlichen Gewichtsabnahme von $1 \mathrm{~kg}$ pro Monat erfolgreich (Ellrott 1994, Ellrott et al. 1998).

Des Weiteren sind die negativen Auswirkungen von höherem Fettverzehr, vor allem in Form von gesättigten Fettsäuren, auf kardiovaskuläre Erkrankungen und bösartige Tumore vielfach untersucht und bestätigt worden (DGE 1996, DGE 2000). Demgegenüber stehen die gesundheitsfördernden Effekte von Vitaminen, Ballaststoffen und sekundären Pflanzenstoffen aus Obst, Gemüse und Vollkornprodukten. Das Prinzip einer Low-fat-Ernährung verbindet diese beiden Vorteile. Es sollen die negativen Auswirkungen der Fette reduziert und die positive Wirkung der vitamin- und ballaststoffreichen Kohlenhydratträger genutzt werden. Aus diesen Überlegungen heraus haben die großen Fachgesellschaften weltweit ihre Empfehlungen für eine gesundheitsfördernde Ernährung abgeleitet: Für die Makronährstoffzusammensetzung der Nahrung wird ein Anteil von < 30\% Fett, ca.10-15\% Eiweiß und > 50\% Kohlenhydrate empfohlen (DGE 2001). Der Definition nach enthält eine fettarme Ernährung < 30\% Fett und damit entsprechen die Empfehlungen einer fettarmen Ernährung.

\subsubsection{Das Konzept des glykämischen Index und der glykämischen Last}

Epidemiologische Daten belegen, dass die Zahl übergewichtiger und adipöser Menschen in den westlichen Industrienationen und bereits darüber hinaus kontinuierlich steigt. Gleichzeitig sinkt der Fettverzehr in der Nahrung der Bevölkerung seit einigen Jahrzehnten, wie aus den Ernährungsstatistiken zu entnehmen ist. Aus diesen Ergebnissen leiten manche Ernährungswissenschaftler einen fehlenden linearen Zusammenhang von Fettverzehr und Gewichtsentwicklung ab. Da der Anteil vor allem von raffinierten Kohlenhydraten in der Nahrung stetig größer wird, postulierte man dagegen einen kausalen Zusammenhang zwischen den mehr verzehrten (raffinierten) Kohlenhydraten und dem steigenden Gewicht der Menschen. Als physiologischen Mechanismus nahm man die unterschiedliche blutglucosesteigernde Wirkung von Kohlenhydraten an. Durch ihre verschiedene Komplexität werden Kohlenhydrate im Körper langsamer oder schneller verdaut. Die Konzepte des glykämischen Index (GI) und der glykämischen Last (GL) basieren auf diesem Effekt. Einfache Kohlenhydrate wie das Monosaccharid Glucose werden ohne Spaltung rasch intestinal resorbiert und sind schnell im Blut nachweisbar. Komplexe Polysaccharide, die zunächst im Darm gespalten werden müssen, bevor sie resorbierbar sind, führen nach ihrer oralen Aufnahme zu einem langsameren Blutglucoseanstieg. Als Referenz des GI wurde die Fläche unter der Glucoseanstiegskurve im Blut 2 Stunden nach oraler Gabe von 50 g Glucose als $100 \%$ festgelegt. Gleiche Mengen anderer kohlenhydrathaltiger Lebensmittel erzeugen einen anderen Blutglucose- 
anstieg innerhalb von 2 Stunden. Dieser wird in Prozent des Anstiegs nach der Glucosegabe beschrieben und als GI für das entsprechende Lebensmittel festgelegt. Raffinierte Kohlenhydrate, beispielsweise Zucker und Weißmehl, besitzen einen hohen GI. Komplexe Kohlenhydrate, aber auch kohlenhydrathaltige Nahrungsmittel mit größerem Fettanteil bewirken einen langsameren Blutglukoseanstieg und ihr GI liegt entsprechend niedriger. Die Insulinausschüttung nach Aufnahme von Nahrungsmitteln mit kleinem GI fällt langsamer und insgesamt geringer aus.

Menschen mit hohem Insulinspiegel lagern Nahrungsfett ein, essen mehr und haben ein höheres Risiko für Übergewicht, Diabetes und Herz-Kreislauferkrankungen (Liu und Willet 2002, Liu et al. 2000, Ludwig 2003). Daher wird vom Verzehr raffinierter Kohlenhydrate mit hohem GI abgeraten, besonders im Rahmen einer Gewichtsreduktion oder zur Vermeidung von Übergewicht. Andererseits werden ,gute“ Kohlenhydrate wie ballaststoffreiches Obst, Gemüse und Vollkornprodukte mit niedrigerem GI, die zudem reich an wichtigen Mikronährstoffen sind, empfohlen.

Der GI ist allerdings für den Alltagsgebrauch unpraktisch, da eine normale Portion eines Lebensmittels nicht unbedingt $50 \mathrm{~g}$ Kohlenhydrate enthält. Daher wurde die glykämische Last (GL) eingeführt. Sie ist der Wert für die glykämische Wirksamkeit einer auf bestimmte Weise zubereiteten Nahrungsportion. Berechnet wird sie durch Multiplikation der Kohlenhydratmenge der Nahrungsportion mit dem entsprechenden GI des Nahrungsmittels geteilt durch 100 (Groeneveld 2004). Zur Vereinfachung von Diäten mit reduzierter glykämischer Last gibt es heute praktische Lebensmitteltabellen, auf denen die GL einer Nahrungsportion nachzulesen ist.

Befürworter dieses Ernährungskonzepts fordern eine generelle Umschichtung der Ernährungspyramide für eine gesunde Ernährung und nicht nur für Reduktionsdiäten (Ludwig 2000). Darin stellen pflanzliche Öle, Obst, Gemüse und Vollkornprodukte die Basis und Zucker und andere raffinierte Getreideprodukte wie Weißmehl und Weißmehlprodukte sowie Kartoffeln die Spitze der Pyramide dar. Zu diesem Konzept gibt es bisher nicht viele und vor allem nur wenige länger dauernde Studien. Beispielsweise konnte eine kurze 10-wöchige Interventionsstudie von Sloth et al. keine bessere Gewichtsabnahme bei Ernährung mit niedriger gegenüber hoher GL finden. Allerdings zeigte sich bei niedriger GL ein leicht positiver Effekt auf die Blutfettparameter (Sloth et al. 2004). 


\subsection{Kardiovaskuläre Risikofaktoren als Gütekriterien einer Diät}

Medizinisch hat sich als Maß für die Güte von Strategien zur Gewichtsreduktion außer der Nettogewichtsabnahme auch die Beeinflussung kardiovaskulärer Risikofaktoren etabliert. So werden neben den Daten des Gewichts in Untersuchungen zum Thema ebenfalls der Blutdruck, der Glucose- und Lipidstatus sowie je nach Fragestellung noch weitere Parameter im Verlauf gemessen. Die positive Entwicklung des Gewichts sollte im besten Fall mit einer Verbesserung aller anderen Risikofaktoren für kardiovaskuläre Erkrankungen einhergehen.

\subsubsection{C-reaktives Protein und hoch sensitives C-reaktives Protein}

Neben den herkömmlichen Risikofaktoren für die Atherosklerose wird zunehmend auch ein Entzündungsmarker, das C-reaktive Protein (CRP), als Maß für das kardiovaskuläre Risiko und die zu erwartende Risikoreduktion unter einer diätetischen Behandlung eingesetzt. Dieses von der Leber synthetisierte pentamere Akute-Phase-Protein wird bei Entzündungen vermittelt durch Interleukin 6 (IL6) sezerniert (Yudkin et al. 1999). Aufgabe des Proteins ist die Erkennung von fremdpathogenen Phosphocholinen sowie von Phospholipiden geschädigter Körperzellen. Durch Anheftung an diese aktiviert es das Komplementsystem und Makrophagen und trägt zum Abbau von Bakterien, Viren, Pilzen oder erkrankten Körperzellen bei. Darüber hinaus kann dieses Protein die Ausschüttung anderer Zytokine induzieren und hat somit seinen festen Platz in Entzündungsprozessen des Körpers.

Während CRP im gesunden Menschen in sehr geringer Menge von bis zu 2 mg/l nachzuweisen ist, kann es bei schwerer körperlicher Arbeit und Stress bereits leicht ansteigen. Bei chronischen Entzündungen im Rahmen einer rheumatoiden Arthritis sind Werte von ca. 5-10 $\mathrm{mg} / \mathrm{l}$ und bei schweren bakteriellen Infektionen bis 300, 400 oder mehr mg/l im Blut zu finden. Mit einer Halbwertszeit von 19 Stunden ist das CRP ein verlässlicher und schneller Marker für die jeweilige Entzündungsaktivität - und das trifft nicht nur für akute, sondern auch chronische Entzündungsprozesse. Mit der Entwicklung von hoch sensitiven Messmethoden mittels eines latex-verstärkten Immunoassays oder ELISA können im Einzelnen Werte bis $0,15 \mathrm{mg} / \mathrm{l}$ gemessen werden. Die auf diese Weise detektierten Werte nennt man entsprechend den Tests auch hoch sensitives CRP oder hsCRP.

Mittlerweile gibt es Hinweise, dass mit diesen neuen Messverfahren sogar die leichte chronische Entzündungsreaktion der Atherosklerose abgebildet werden kann. Ein Zusammenhang von CRP und Atherosklerose wurde durch den Nachweis von CRP in atherosklerotischen Läsionen des Endothels gefunden. Weitere Hinweise lieferten große Kohorten-Studien 
wie die Framingham Offspring Study. Durch sie weiß man, dass Menschen ohne anderweitige Erkrankungen, aber mit einem leicht erhöhten hsCRP-Wert ein erhöhtes Risiko für ein kardiovaskuläres Ereignis in den nächsten Jahren haben. Das Risiko steigt mit steigendem hsCRPWert an (Rutter et al. 2004). Dieser lineare Zusammenhang stellt sich in einigen Studien so eindeutig dar, dass er gemeinsam mit dem Lipidscreening zur Einstufung des kardiovaskulären Risikos bei Menschen mit Atherosklerose, aber auch bei ansonsten scheinbar gesunden Menschen genutzt werden kann (Nader und Ridker 2001a, Nader und Ridker 2001b).

Andere Studienergebnisse zeigen eine positive Korrelation von hsCRP und BMI (Ford 1999, Tracy 2001). Erklärbar ist dieser Zusammenhang durch die Stoffwechselaktivität des viszeralen Fettgewebes. Durch die Sezernierung von biologisch aktiven Proteinen wie IL6 und Tumornekrosefaktor $\alpha$ (TNF $\alpha$ ) durch die Fettgewebszellen wird ein leichter Entzündungsprozess in Gang gesetzt. Als Folge der IL6-Freisetzung wird CRP produziert. Die Zusammenhänge zwischen Adipositas, ihren Folgeerkrankungen und einem geringen Entzündungsprozess sind auf molekularbiologischer Ebene in einem Artikel von Guerre-Millo im Diabetes Metabolism bereits 2004 anschaulich dargestellt worden (Guerre-Millo 2004).

Eine hypothetische Folgerung aus den beobachteten Zusammenhängen von hsCRP und BMI hat sich bisher ebenfalls als richtig erwiesen: Wenn bei erhöhtem BMI ein erhöhter hsCRP-Wert vorliegt, sollte dieser bei Gewichtsreduktion und fallendem BMI sinken. Genau dieser Abfall im CRP-Spiegel ließ sich in einigen Studien unter Gewichtsreduktion belegen (Esposito et al. 2003, Dansinger et al. 2005). Da es allerdings verschiedene Einflüsse auf das CRP/ hsCRP gibt, ist eine Aussage über den genauen Mechanismus dieses Effekts zurzeit noch schwierig. Mehrere Ursachen wären denkbar: Eine Abnahme des Fettgewebes verringert die Produktion des CRP-Induktors IL6 und damit den hsCRP-Spiegel. Oder eine gesündere Ernährung und Lebensweise führt zur Gewichts- und zur CRP-Reduktion. Der Fakt, dass tierisches Fett und Fleisch die Vorstufe der proinflammatorischen Prostaglandine liefern, und eine ausgewogene Ernährung üblicherweise wenig davon enthält, ließe auch diese zweite Interpretation zu (Adam 2003). Erkenntnisse aus der Ernährungstherapie der chronischen Polyarthritis, einer chronisch entzündlichen Erkrankung, geben Hinweise für positive Auswirkungen bestimmter Ernährungsarten auf entzündliche Prozesse: Eine Polyarthritis bessert sich unter einer konsequent vegetarischen Kost, die keine Arachidonsäure als Vorstufe proinflammatorischer Prostaglandine enthält (Kjeldsen-Kragh et al. 1991).

Wegen der verschiedenen Einflussfaktoren auf das CRP ist eine hsCRP-Messung auch besonders anfällig für Überlagerungen durch entzündliche Prozesse jeglicher Art. Normaler- 
weise ist der hsCRP-Wert eines Menschen über einen längeren Zeitraum stabil, das heißt, er fällt nach einem akuten Infekt relativ schnell wieder auf sein vorheriges Niveau ab (Macy et al. 1997). Wenn erhöhte Werte gemessen werden, kann eine Wiederholung der Messung nach 2-3 Wochen Aufschluss darüber bringen, ob ein akutes entzündliches Geschehen dem zugrunde gelegen hat oder - bei fehlender Normalisierung des Wertes - eben nicht. Bleibt der hsCRP-Wert in einer Kontrolle stabil leicht erhöht, kann daraus auf das mögliche Vorliegen eines chronischen Entzündungsgeschehens geschlossen werden, zum Beispiel auf eine Atherosklerose.

\subsubsection{Homocystein - kardiovaskulärer Risikofaktor, Marker des Vitamin- status oder beides?}

Neben den etablierten Risikofaktoren der Atherosklerose ist in den letzten Jahren die Erhöhung des Homocysteinspiegels als Mitverursacherin der Atherosklerose diskutiert und untersucht worden. Schon 1969 hat McCully erste Beobachtungen über den Zusammenhang der sehr frühen Entstehung einer arteriellen Verschlusskrankheit bei Kindern mit homozygoter Homocystinurie beobachtet und Homocystein als entscheidende Ursache der frühen Erkrankung beschrieben. Erst seit Beginn der 1990er Jahre gelang es dann, die Rolle der Aminosäure Homocystein im Stoffwechsel genauer zu verstehen (McCully1996).

Untersuchungen zur Wirkung von Homocystein liefern dabei Hinweise für das direkt schädigende Potential dieser nicht proteinogenen Aminosäure. Sie entsteht im Zellstoffwechsel beim Abbau von Methionin und wird als Methylgruppendonator an der Schnittstelle zwischen Methionin- und Folsäurezyklus benötigt. Durch den permanenten Um- und Abbau von Homocystein liegen beim stoffwechselgesunden Menschen normalerweise keine größeren Mengen an Homocystein im Blut vor. Bei einem Mangel an Folsäure, Vitamin B6 oder B12 kommt es wie auch bei einem Mangel an funktionstüchtigen Enzymen des Homocysteinabund -umbaus zu einer Kumulation von Homocystein. Die wichtigsten Enzyme diesbezüglich sind die 5,10-Methylen-Tetrahydrofolat-Reduktase (MTHFR) und die Cystathionin- $\beta$ Synthase (CBS). Das Ineinandergreifen von Folsäure- und Methioninzyklus und die Einwirkung der verschiedenen Vitamine wird in dem Buch „Homocysteine and vascular disease“ auch schematisch gut dargestellt: 


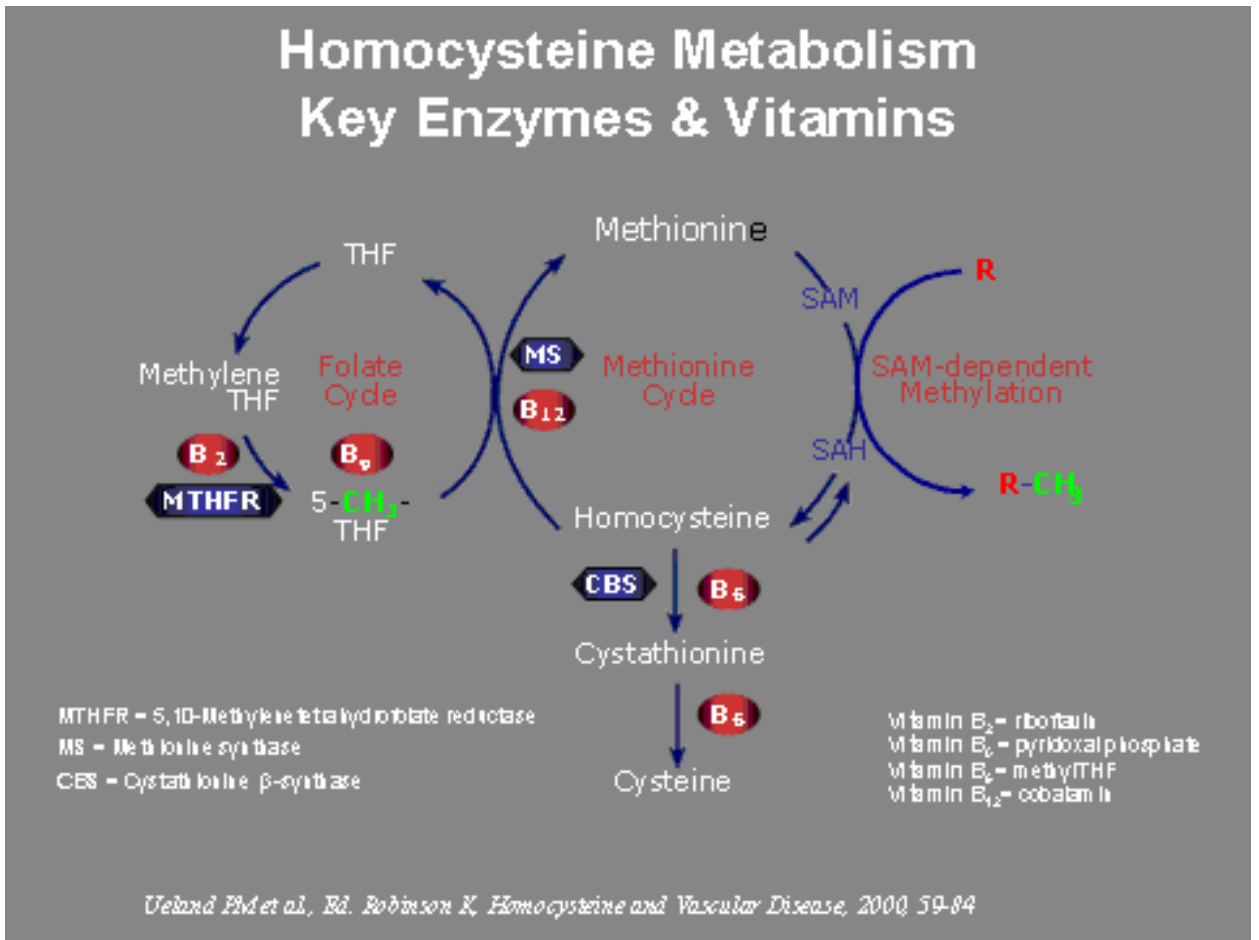

$\mathrm{THF}=$ Tetrahydrofolat

Methylen-THF $=5,10$-Methylen-Tetrahydrofolat

CBS $=$ Cystathionin $-\beta-$ Synthase

SAM $=$ S-Adenosyl-Methionin

B 2 = Vitamin B2 (Riboflavin)

B 9 = 5-Methylen-Tetrahydrofoat
5-CH3-THF = 5-Methyl-Tetrahydrofolat MTHFR $=5,10$-Methylen-Tetrafolat-Reduktase MS = Methionin-Synthase

$\mathrm{SAH}=$ S-Adenosyl-Homocystein

B $6=$ Vitamin B6 (Pyridoxalphosphat)

B 12 = Vitamin B12 (Cobalamin)

Abbildung 1: Homocysteinstoffwechsel (Ueland et al. 2000 b)

Eine Hyperhomocysteinämie kann in den arteriellen Gefäßen und im Gerinnungssystem Schäden verursachen und sich negativ auf Neuralrohrentwicklung, Geburtsverlauf und die kognitive Leistungsfähigkeit auswirken (Ueland et al. 2000 a). Das multiple toxische Potential von Homocystein auf das arterielle System besteht einerseits in seiner Fähigkeit, direkt Läsionen in der Gefäßinnenhaut zu bewirken, und indirekt, durch seine Oxidation reaktive Sauerstoffradikale freizusetzen, die ihrerseits das Endothel angreifen. Über eine Verschiebung des Gleichgewichts im aktivierten Methyl-Zyklus der Zelle kann eine Hyperhomocysteinämie zudem die Endothelzellentwicklung behindern. Die Störung der Endothelzellentwicklung bei ungehinderter Proliferation der glatten Gefäßmuskulatur durch einwandernde Muskelzellen und Wachstumsfaktoren begünstigt die Entstehung atherosklerotischer Plaques (Medina et al. 2001). Diese Untersuchungsergebnisse stützen die Hypothese, dass Homocystein ein unabhängiger Risikofaktor für Atherosklerose und kardiovaskuläre Erkrankungen ist. Aus den Daten des europäischen COMAC-Projekts kann entnommen werden, dass eine Hyperhomocysteinämie die Effekte der anderen artherosklerotischen Risikofaktoren sogar multiplizieren kann (Graham et al. 1997). 
Die wissenschaftliche Debatte um das schädigende Potential von Homocystein und seinen Einfluss auf andere Erkrankungen ist allerdings noch nicht abgeschlossen. Die Kontroverse wird durch die Möglichkeit angeregt, eine Hyperhomocysteinämie könne ebenso wie die Atherosklerose eine Folge der bekannten Risikofaktoren sein und damit nur ein Marker für atherosklerotische Erkrankungen (Brattström und Wilcken 2000). Aus der Tatsache, dass der Homocysteinspiegel so gut mit den Blutspiegeln von Vitamin B6, B12 und Folsäure korreliert, dass er als Marker für einen Mangel dieser Vitamine in Forschung und Klinik eingesetzt werden kann (Refsum et al. 2004), ergibt sich noch eine andere Überlegung. Ein Mangel dieser Vitamine könnte die Schäden in den Gefäßen und bei der Neuralrohrentwicklung etc. verursachen und gleichzeitig eine Homocysteinerhöhung als Epiphänomen bewirken (Verhoef und Katan 2004). Bisher konnte diese Hypothese jedoch auch nicht eindeutig bestätigt werden.

Weitere Untersuchungen zum Thema Homocystein beschäftigten sich mit genetischen Defekten, die zu einer Hyperhomocysteinämie führen können. Der häufigste Gendefekt, der negativ in den Homocysteinkreislauf eingreift, ist eine Punktmutation des Gens für die MTHFR. Bei Austausch von C durch T am Nukleotid $677(\mathrm{C} 677 \rightarrow \mathrm{T})$ des entsprechenden Gens entsteht eine thermolabile Enzymvariante mit eingeschränkter Aktivität. Dadurch wird zuwenig Methyl-Tetrahydrofolat als Methylgruppendonator für den Abbau von Homocystein zu Methionin bereitgestellt. Die Genmutation äußert sich vor allem bei homozygoten Trägern und bei gleichzeitigem Mangel an den oben genannten Vitaminen in einer deutlichen Hyperhomocysteinämie (Medina et al. 2001). Andere genetische Defekte betreffen unter anderem die CBS. Ein eigener Krankheitswert über die Erhöhung des Homocysteinspiegels hinaus konnte diesen Gendefekten allerdings nicht nachgewiesen werden. Andere Faktoren, die den Homocysteinspiegel anheben, wie starker Kaffeekonsum, Gewichtsabnahme, Alter und männliches Geschlecht besitzen ebenso wenig ein Krankheitspotential an sich (Nurk et al. 2004).

Aus dem eindeutigen Zusammenhang der Vitaminspiegel von Folsäure, Vitamin B6 und B12 mit dem Homocysteinspiegel hat in den letzten Jahren die erfolgreiche Empfehlung zur Senkung des Homocysteins im Blut mittels Vitaminsupplementation geführt. In kontrollierten Vergleichsstudien wurde das mögliche Präventionspotential einer Homocysteinsenkung in der Primär- und Sekundärprävention atherosklerotischer Erkrankungen untersucht. Die Ergebnisse der Untersuchungen sind uneinheitlich. In einigen dieser Arbeiten wurden signifikant positive Effekte zum Beispiel auf die Intima-Media-Dicke der Karotiden durch Senkung des Homocysteins entdeckt (Marcucci et al. 2003). Die Studiendaten einer weiteren Untersuchung 
konnten eine Reduktion der Re-Stenoserate nach PTCA unter Folsäure-, Vitamin B6- und B12-Substitution zeigen (Schnyder et al. 2001), während in den 2006 veröffentlichten Ergebnissen der HOPE 2-Studie keine Effekte gefunden wurden (Lonn et al. 2006). Im Norwegian Vitamin Trial (NORVIT) ergaben sich sogar negative Auswirkungen der Vitaminsubstitution (Bonaa et al. 2006). Empfehlungen zum Einsatz von Vitaminpräparaten zur Homocysteinsenkung nach koronarer Intervention, also in der Sekundärprävention, können momentan daher nicht gegeben werden. Für die Einschätzung von Homocystein als kardiovaskulärem Risikofaktor und Marker für einen Mangel an Vitamin B6, B12 und Folsäure gibt es dagegen heute genug Daten. Und diese beiden Punkte machen Homocystein zu einem interessanten Parameter in der Beurteilung von diätetischen Interventionen.

\subsection{Das Ziel dieser Studie}

Bisherige Aussagen zur Güte und Sicherheit diverser populärer Diätstrategien stammen überwiegend aus den USA und Kanada, besonders die zu kohlenhydratarmen Diäten. Unter deutschen Lebensbedingungen wurden bisher noch keine Untersuchungen zu den aktuell diskutierten Diätstrategien durchgeführt, obwohl sie, wie Atkins Diät-Revolution und so genannte Glyx-Diäten mit reduzierter glykämischer Last, auch in Deutschland bereits stark vermarktet werden. Da sich die Ernährungsgewohnheiten und die Nahrungsangebote in den USA/Kanada und Deutschland in einigen Punkten deutlich unterscheiden, sind relevante Auswirkungen für die Durchführbarkeit und die Ergebnisse dieser Diätstrategien in Deutschland denkbar: Erstens sind Getreideprodukte in den USA und Kanada nach einer Vorschrift mit Folsäure angereichert und in Deutschland nicht. Folsäure zählt zu den kritischen Vitaminen, deren ausreichende Aufnahme unter hiesigen Lebensbedingungen nicht unbedingt gesichert ist (DGE et al. 2000). Das kann besonders unter den Bedingungen einer Reduktionsdiät zu einem klinisch relevanten Folsäuremangel führen. Zweitens gibt es in Bezug auf Low-carbDiäten einen wichtigen Unterschied zwischen den USA und Deutschland, was das Nahrungsangebot betrifft: In vielen US-amerikanischen Supermärkten werden Low-carb-Produkte angeboten und hierzulande selten. Damit ist das Lebensmittelangebot für Low-carb-Diäten in Deutschland weitaus stärker eingeschränkt als dort, wo die meisten Studien zu Low-carbDiäten bisher durchgeführt wurden. Diese Tatsachen unterstützen die Notwendigkeit einer Untersuchung der Auswirkungen und Anwendbarkeit von Low-carb- und Glyx-Diäten unter deutschen Lebensbedingungen. 
Unsere Studie soll erste Vergleiche zu Wirksamkeit und Sicherheit verschiedener Diätvarianten unter deutschen Lebensbedingungen ermöglichen. Zu diesem Zweck werden neben Gewicht, Bauchumfang und Blutdruck auch verschiedene metabolisch aussagekräftige Serumparameter wie Lipide, Glucose, Harnsäure, hsCRP, Leber- und Nierenwerte und Homocystein als Marker für den Vitaminstatus und als eigenständiger kardiovaskulärer Risikofaktor zu Beginn und im Verlauf der Diätinterventionen untersucht.

\subsubsection{Das Studiendesign}

Zum Vergleich der drei Diätansätze Low carb (LC), Low fat (LF) und Low fat with reduced glycemic load (LFRGL) wurde das Modell einer offenen klinischen Vergleichsstudie gewählt. Als Probandinnen wurden übergewichtige bis mäßig adipöse, weitgehend gesunde Frauen gesucht, die in den letzten 6 Monaten keinen Diätversuch unternommen hatten. Die 160 Teilnehmerinnen wurden nach BMI und Alter randomisiert den drei diätetischen Gruppen zugeteilt. Vor der Zuordnung zu einer Diätgruppe erfolgte eine Ernährungsbefragung mit dem Computerprogramm Meditouch, um die alltägliche Ernährungsweise vor der Diät festzustellen. Diese Ernährungserhebung wurde nach 6 Monaten Diät wiederholt.

Alle drei diätetischen Interventionen basieren auf einer aktuell diskutierten Ernährungsstrategie zur Gewichtsreduktion. In jeder Diätvariante wird ein bestimmter Makronährstoff wie Kohlenhydrate oder Fett in eingeschränktem Maß verzehrt. Die anderen Makronährstoffe können in freier Menge zu sich genommen werden. Die Beschränkung des bestimmten Makronährstoffs wurde jeweils in drei Phasen gestaffelt. In der strengen Anfangsphase mussten die Probandinnen für 4 Wochen mit jeweils $30 \mathrm{~g}$ Kohlenhydraten oder $30 \mathrm{~g}$ Fett auskommen beziehungsweise mit $30 \mathrm{~g}$ Fett und zusätzlich maximal $50 \mathrm{~g}$ Kohlenhydraten mit hoher GL. Die Beschränkung der Kohlenhydrate mit hoher GL blieb in allen drei Diätphasen der Gruppe LFRGL gleich. An die erste Phase schloss sich ein 5-monatiger Abschnitt mit 60 g Kohlenhydraten beziehungsweise $45 \mathrm{~g}$ Fett an. Anschließend folgte eine Erhaltungsphase mit bis zu $90 \mathrm{~g}$ Kohlenhydraten beziehungsweise $60 \mathrm{~g}$ Fett. Die vorliegende Arbeit beschränkt sich auf die Auswertung der ersten 6 Monate der Intervention.

Während dieser ersten 6 Monate kamen die Teilnehmerinnen wöchentlich zum Wiegen und bekamen schriftliche Motivationshilfen. Anschließend gab es monatliche Wiegetermine für die weiteren 6 Monate. Die Blutuntersuchungen, Messungen des Blutdrucks, Pulses und Bauchumfangs fanden vor der Intervention, nach 4 Wochen und nach 6 Monaten statt. So konnten die Effekte der einzelnen Ernährungsphasen kontrolliert werden. 
Eine Erhebung zu den Lebensumständen, dem Wohlbefinden und zur Zufriedenheit mit der Diät wurde ebenfalls durchgeführt, wird jedoch gesondert ausgewertet. Sie ist daher nicht Teil dieser Arbeit.

\subsection{Der Ethikkommissionsantrag}

Diese Vergleichsstudie verschiedener diätetischer Strategien zur Gewichtsreduktion arbeitet mit freiwilligen Teilnehmerinnen und verschiedenen Interventionsarmen. Sie unterliegt damit der Zustimmung der Ethikkommission. Die Zustimmung wurde von der Ethikkommission der Medizinischen Fakultät der Universität Göttingen unter der Auftragsnummer 32/11/2004 erteilt. 


\section{Probandinnen, Material und Methoden}

\subsection{Probandinnen}

\subsubsection{Rekrutierung der Probandinnen}

Zur Rekrutierung der Probandinnen wurde ein Zeitungsartikel in der örtlichen Presse veröffentlicht. Er erschien am 16.01.2005 im wöchentlichen Anzeigenblatt Extra Tip und am 21.01.2005 in der Tageszeitung Göttinger Tageblatt. Übergewichtige Menschen mit Interesse an einer Gewichtsreduktion wurden darin aufgefordert, sich telefonisch zu angegebenen Zeiten in der Ernährungspsychologischen Forschungsstelle zu melden.

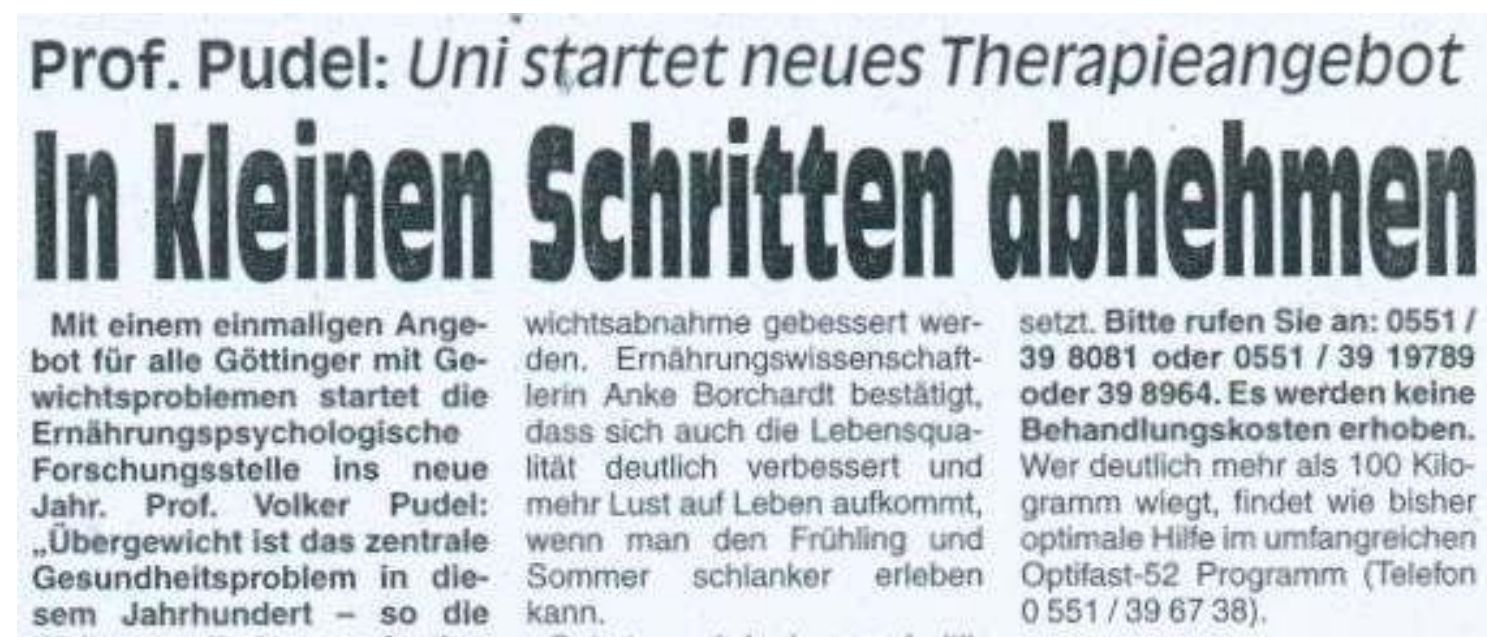
Weltgesundheitsorganisation WHO. Unser Team bietet ab sofort in mehreren Programmen wirksame Behandlung an".

Frauen und Mắnner ùber 18 Jahre kônnen sich anmelden, wenn sie zwischen fünt und 15 Kilogramm abnehmen wollen.

Dr. Thomas Ellrott, der die Eingangsuntersuchungen leitet, weiß, wie positiv Risikofaktoren, zum Beispiel erhohter Blutdruck, Diabetes oder Gelenkbe. schwerden, durch eine Ge-

wichtsabnahme gebessert werden. Ernahrungswissenschaftlerin Anke Borchardt bestătigt, dass sich auch die Lebensqualitat deutlich verbessert und mehr Lust auf Leben aufkommt, wenn man den Frohling und Sommer schlanker erleben Geboten wird ein ganzheitliches Programm fúr ein geàndertes Essverhalten und aktiven Lebensstil, das den Essgenuss bewahrt und Ubergewicht abbaut. „Wir bieten natürlich keine kurzfristige Schiankheitsdiat an, denn die macht eher dick als dün. In kleinen Schritten wird geübt, von Woche zu Woche", verspricht Protessor Pudel (Foto), der mit seinem Team seit 35 Jahren wissenschaftliche Grundlagen für die Ubergewichtstherapie erforscht,

$\mathrm{Ab}$ sofort sind die Teletone be-

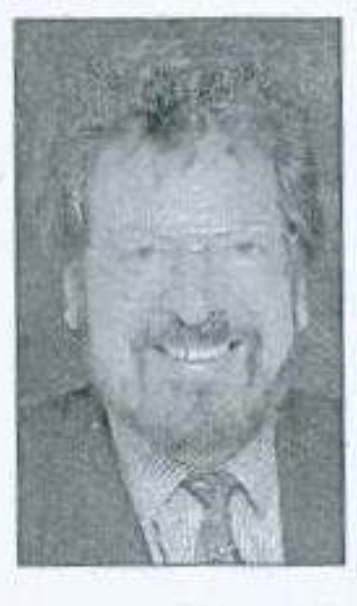

Abbildung 2: Zeitungsartikel aus dem „Extra Tip“ vom 16.01.2005 (Extra Tip 2005)

In den darauf folgenden Tagen riefen über 230, überwiegend weibliche Kandidaten an. 


\subsubsection{Auswahl der Probandinnen}

In diesem ersten Telefonkontakt mit den potentiellen Probandinnen wurden mithilfe eines strukturierten Interview-Leitfadens bereits die wichtigsten Kriterien für eine mögliche Teilnahme an dem Programm abgefragt. Wenn sich dabei keine Einwände gegen eine Teilnahme entnehmen ließen, wurden den Kandidatinnen Fragen beantwortetet und die wichtigsten Informationen zum Programm mit zeitlichem Ablauf weitergegeben.

Sie bekamen Informationen über:

1. die Kostenfreiheit des Programms

2. die körperliche Eingangsuntersuchung

3. den möglichen Ausschluss vom Programm beim Bestehen oder Auftreten von Ausschlusskriterien auch während des laufenden Programms

4. die geplanten drei Nüchtern-Blutabnahmen zur Kontrolle von Stoffwechselparametern

5. die Aufteilung aller Teilnehmerinnen auf drei Diätgruppen, die sie selbst nicht beeinflussen können

6. den Ablauf des Programms mit wöchentlichen Gewichtskontrollen für 6 Monate, anschließend monatlichen Gewichtskontrollen bis zu einem Jahr jeweils in der Ernährungspsychologischen Forschungsstelle

7. die Ernährungsempfehlungen mit unterschiedlich strengen Vorgaben für die gestaffelten Phasen der Diätinterventionen

8. die Form des Programms mit rein schriftlichen Diätanweisungen ohne Individualoder Gruppensitzungen

9. die zu erwartende Gewichtsabnahme von bis zu ca. 5-15 Kilogramm

10. die Möglichkeit der Programmbeendigung zu jedem beliebigen Zeitpunkt seitens der Teilnehmerinnen

11. die wissenschaftliche Begleitung des Programms zur Evaluierung der Ernährungsstrategien, ihrer Auswirkungen auf den Stoffwechsel und der Teilnehmerinnenzufriedenheit.

Im Anschluss an das Telefonat erhielten die Kandidatinnen Informationsbriefe mit Rückumschlag. Darin waren eine Teilnahmeinformation, je ein Fragebogen zur medizinischen Anamnese, zum Essverhalten (FEV) und zur gesundheitsbezogenen Lebensqualität (SV 36) sowie die Einverständniserklärung zur Teilnahme an dem Therapieprogramm enthalten. 
Wenn die Informationen aus den Fragebögen eine Aufnahme in das Untersuchungsprogramm ermöglichten, erfolgte telefonisch eine Terminvereinbarung mit den Kandidatinnen für eine Eingangsuntersuchung und die erste Blutentnahme. Dieser Termin wurde anschlieBend schriftlich bestätigt.

Die ärztliche Untersuchung bestand aus einer Blutdruck- und Pulsmessung, der Messung von Größe und Gewicht mit Berechnung des BMI, der ersten Blutabnahme sowie einer klinischen Inspektion mit Herz- und Lungenauskultation. Die medizinische Anamnese wurde im Gespräch vervollständigt. Nach Einverständnis der jeweiligen Probandin ergänzten eventuelle Rücksprachen mit den behandelnden Haus- und Fachärzten und Befunde von Voruntersuchungen die Statuserhebung. All diese Informationen inklusive der Ergebnisse der ersten Blutuntersuchung gingen als Kriterien in die letztendliche Auswahl der Teilnehmerinnen ein.

\subsubsection{Ausschlusskriterien}

Die Aufnahme in das Programm war abhängig von den folgenden Ausschlusskriterien:

1. männliches Geschlecht

2. $\quad$ BMI $<25$ und $>42 \mathrm{~kg} / \mathrm{m}^{2}$

3. Alter $<25$ und $>70$ Jahre

4. Schwangerschaft und Stillzeit

5. Diäten mit Gewichtsreduktion in den letzten 6 Monaten

6. schwerere akute und/ oder chronische Erkrankungen

7. insulinpflichtiger Diabetes mellitus

8. Kreatininwert $>1,5 \mathrm{mg} / \mathrm{dl}$ in der ersten Blutabnahme

9. Verweigerung einer schriftlichen Einverständniserklärung zur Teilnahme an dem Programm mit anonymisierter wissenschaftlicher Auswertung der erhobenen Daten.

$\mathrm{Zu}$ 1. Die überwiegende Mehrheit von Diäten wird von Frauen durchgeführt. Daher haben wir zur Untersuchung der verschiedenen Ernährungsstrategien nur die vorrangig betroffene Bevölkerungsgruppe einbezogen. Zudem wären geschlechtsspezifische Unterschiede erst bei einer deutlich größeren Zahl von Teilnehmerinnen und Teilnehmern aussagekräftig geworden.

$\mathrm{Zu}$ 2. Menschen mit einem BMI $<25 \mathrm{~kg} / \mathrm{m}^{2}$ sind nicht übergewichtig oder adipös (WHO 2005). Sie bedürfen keiner Gewichtsreduktion. Personen mit Übergewicht (BMI 25$29,9 \mathrm{~kg} / \mathrm{m}^{2}$ ) dagegen können und mit einer Adipositas I (BMI 30-34,9 kg/m²) oder höher soll- 
ten eine Reduktion ihres Körpergewichts anstreben, um adipositas-assoziierte Gesundheitsstörungen zu vermeiden oder zu verringern (Hauner et al. 2005). Für die ambulante und überwiegend ernährungsbezogene Therapie zur Beeinflussung ihres Körpergewichts sind Menschen mit Übergewicht oder leichter bis mäßiger Adipositas zudem am ehesten geeignet.

Eine Frau mit einem BMI von knapp unter $25 \mathrm{~kg} / \mathrm{m}^{2}$, genau 24,6 kg/m² haben wir trotz Erfüllen dieses Ausschlusskriteriums mit in das Programm aufgenommen, da sie wegen ihres Bauchumfangs von $92 \mathrm{~cm}$ ebenfalls ein metabolisches Risikoprofil besaß.

$\mathrm{Zu}$ 3. Aufgrund des großen Universitätsstandortes Göttingen sind Erwachsene unter 25 Jahren überdurchschnittlich häufig Studierende. Um einen aussagekräftigen Querschnitt durch die Normalbevölkerung im Teilnehmerinnenfeld zu haben, wurde die Gruppe der unter 25Jährigen ausgeschlossen.

$\mathrm{Zu}$ 4. Eine Gewichtsreduktion in Schwangerschaft und Stillzeit ist medizinisch nicht empfehlenswert und kann gesundheitliche Schäden für Mutter und Kind hervorrufen.

$\mathrm{Zu}$ 5. Durch eine vorangegangene Gewichtsreduktion in den letzten Monaten könnten sich unklare Auswirkungen auf die aktuelle Diätintervention ergeben.

Zu 6. Mit schwereren Erkrankungen sind Gesundheitsstörungen gemeint, die eine engmaschigere Kontrolle im Falle einer Gewichtsreduktion bedürfen oder die untersuchten Laborwerte beeinflussen können. Darunter fallen fortgeschrittene KHK, aktuelle Krebserkrankungen, Leber- und Nierenerkrankungen, alle chronisch-entzündlichen Erkrankungen und insgesamt alle Krankheiten, die eine normale Lebensführung mit normaler Ernährung deutlich beeinträchtigen.

Zu 7. Ein insulinpflichtiger Diabetiker benötigt bei der Gewichtsreduktion eine engmaschige Blutzuckerkontrolle, die durch die eingeschränkte medizinische Betreuung in diesem Programm nicht zu gewährleisten ist.

Zu 8. Für Menschen mit Niereninsuffizienz ist eine eiweißreduzierte Kost indiziert. Eine Low-carb-Diät entspricht diesen Kriterien nicht.

Zu 9. Eine schriftliche Einverständniserklärung zur Teilnahme an dem Programm mit anschließender Datenverwendung zu Studienzwecken wird durch die Ethikkommission gefordert. 


\subsubsection{Verteilung auf die Diätgruppen}

Durch das Auswahlverfahren wurden 160 Teilnehmerinnen selektiert. Zur Verteilung auf die 3 unterschiedlichen Diätgruppen wurde eine Randomisierung mit anschließender Schichtung nach Alter und BMI durchgeführt. Eine Berücksichtigung persönlicher Präferenzen der Teilnehmerinnen für eine bestimmte Diät fand dabei ausdrücklich nicht statt.

\subsection{Materialien}

\subsubsection{Blutentnahmen}

Mit der schriftlichen Bestätigung des Termins für die Eingangsuntersuchung und die erste Blutentnahme wurden die Teilnehmerinnen darauf hingewiesen, dass sie nach $22.00 \mathrm{Uhr}$ des Vorabends und bis zur Blutentnahme nichts essen und trinken dürfen. Nur die Einnahme von Blutdruckmedikamenten mit einem kleinen Schluck Wasser wurde zur Vermeidung von Blutdruckentgleisungen erlaubt. Alle Blutentnahmen fanden in sitzender Position aus einer Armvene mit vorheriger Hautdesinfektion statt.

Zur Venenpunktion wurden Monovettenkanülen mit 0,8 mm Durchmesser benutzt. Für die Bestimmung der unterschiedlichen Laborparameter war bei jeder Blutentnahme pro Probandin die Entnahme von $10 \mathrm{ml}$ Serum erforderlich. Beim ersten Termin sowie zur Bestimmung des MTHFR-Genotyps in der Zwischenblutentnahme für 40 Probandinnen erfolgte zusätzlich die Entnahme von 2 ml EDTA-Blut.

\subsubsection{1. Blutentnahme}

Die 1. Blutentnahme diente nicht nur der Bestimmung der metabolischen Ausgangsparameter sondern auch dem Screening auf Ausschlusskriterien für die Teilnahme. Die Bestimmung des Blutbildes sollte Veränderungen der Anzahl von Blutteilchen aufdecken helfen. Darüber hinaus wurde die Schilddrüsenfunktion als eine mögliche Variable in der Genese von Übergewicht und Adipositas überprüft. Nieren-, Leber- und Harnsäurewerte sollten weitestgehend normal sein, um Risiken bei der Durchführung bestimmter Diäten auszuschließen (siehe 2.1.3 Ausschlusskriterien).

In den beiden Wochen vom 14. bis 18.02. und vom 21. bis 26.02.2005 fanden die ersten Blutentnahmen jeweils in der Zeit von 6.30 bis 11.00 Uhr statt. Bei diesem ersten Termin in der Ernährungspsychologischen Forschungsstelle für die Teilnehmerinnen fand auch die ärztliche Anamnese und Untersuchung statt. 


\subsubsection{2. Blutentnahme}

Die 2. Blutabnahme erfolgte in den Tagen vom 04. bis 08.04. und vom 11. bis 15.04.2005 wiederum nüchtern. Die Uhrzeiten waren wie beim ersten Termin. Es wurden Blutdruck, Puls, Gewicht und Bauchumfang kontrolliert.

\subsubsection{Zwischenblutentnahme}

Wegen Auffälligkeiten bei den Homocysteinwerten einiger Teilnehmerinnen in der 2. Blutentnahme wurde die Kontrolle dieses Parameters vorgezogen. In der Zeit vom 06. bis 24.06.2005 wurde eine gesonderte Blutentnahme bei allen Teilnehmerinnen mit einem Anstieg der Homocysteinwerte von mehr als $5 \mu \mathrm{mol} / \mathrm{l}$ und bei allen Teilnehmerinnen der kohlenhydratarmen Diätgruppe vorgenommen.

Zur Klärung eines möglichen Zusammenhangs zwischen dem Homocysteinanstieg und dem Vorhandensein einer genetischen Variante des für das Enzym MTHFR codierenden Gens ließen wir zusätzlich eine MTHFR-Genotypisierung bei diesen Teilnehmerinnen durchführen. Das schriftliche Einverständnis für die Genotypbestimmung war zuvor von allen betroffenen Teilnehmerinnen eingeholt worden.

\subsubsection{4. Blutentnahme}

Um den Einfluss der unterschiedlichen Ernährungsregime im Verlauf zu untersuchen, wurden die Laborparameter erneut nach 6 Monaten kontrolliert. Die Blutentnahme wurde in der Zeit vom 12. bis 16.09. und vom 19. bis 23.09.2005 durchgeführt, ebenfalls in der Zeit von 6.30 bis 11.00 Uhr. Wegen Urlaubszeiten wurde einzelnen Teilnehmerinnen ein gesonderter Termin bis spätestens zum 21.10.2005 ermöglicht. Auch bei dieser Blutentnahme wurden Blutdruck, Puls, Gewicht und Bauchumfang bestimmt.

\subsubsection{Therapieprogramm}

\subsubsection{Allgemeiner Teil}

Das gesamte Therapieprogramm war für eine mäßige Gewichtsreduktion im ambulanten Rahmen konzipiert. Analog zu den Empfehlungen der Fachgesellschaften wurde das Programm unter medizinischer Aufsicht durchgeführt und enthielt Empfehlungen zur Ernährungs- und Bewegungstherapie sowie verhaltenstherapeutische Elemente. $\mathrm{Zu}$ allen 3 Therapiebausteinen gab es Informationen, Anweisungen und Empfehlungen in rein schriftlicher Form. 
Zu Beginn der Diätphase erhielten alle Teilnehmerinnen ein mehrseitiges Informationspapier mit Beschreibung ihrer jeweiligen diätetischen Therapie. Im Rahmen von wöchentlichen Hand-Outs wurden zusätzlich Anregungen zur kreativen Diätausgestaltung mit Rezeptvorschlägen verteilt. In der Gewichtsstabilisierungsphase nach 6 Monaten Diät wurden für ein weiteres halbes Jahr monatliche Anschreiben an die Teilnehmerinnen ausgehändigt. Die Texte zur Ernährung in diesen Hand-Outs waren jeweils der entsprechenden Lebensmittelauswahl der Diätgruppen angepasst.

Die Anschreiben enthielten darüber hinaus Anregungen zur Bewegungstherapie mit konkreten Übungen und Beispielen (10 Minuten Fitness-Übungen, Nordic-Walking, Treppen steigen statt Fahrstuhl und anderes). Als Elemente der Verhaltenstherapie dienten Texte zu Themen wie „flexible Kontrolle“ als psychologisches Instrument oder Beiträge zum Umgang mit Misserfolgen. Diese Texte sollten die Motivation fördern und den Erfolg des Programms unterstützen.

Zur Ausschaltung von systematischen Fehlern zwischen den unterschiedlichen Gruppen waren die allgemeinen Informationen für alle Teilnehmerinnen exakt gleich. Um auch die so genannte Therapeuten-Variable, die Auswirkung von Interaktionen zwischen Teilnehmerinnen und Betreuern, zu minimieren, wurde zur Motivationsförderung und Informationsweitergabe diese schriftliche Form gewählt. Wenn Teilnehmerinnen Fragen oder Probleme mit dem Programm oder der Diät hatten, bestand dennoch die Möglichkeit der Klärung im direkten Gespräch in der Ernährungspsychologischen Forschungsstelle.

\subsubsection{Ernährungstagebuch}

Nach der endgültigen Auswahl und vor der Zuordnung zu einer Diätgruppe, führte jede Teilnehmerin ein 7-Tage-Protokoll als Ernährungstagebuch. Dieses in der Ernährungswissenschaft etablierte Verfahren zur Erfassung der Ernährungsgewohnheiten diente hierbei als psychologisches Schulungselement für die Teilnehmerinnen. Die Protokollierung sollte der Bewusstwerdung der eigenen Essgewohnheiten dienen (Keil 2003). Darüber hinaus war die Protokollierung eine Vorbereitung auf die Phase der Ernährungsintervention, in der Lebensmittelmengen abgeschätzt und aufgeschrieben werden sollten.

\subsubsection{Punkte zur Selbstkontrolle}

Zur Selbstkontrolle für die Teilnehmerinnen wurden Hefte und Karten mit Punkten eingesetzt. Ein Punkt entsprach $1 \mathrm{~g}$ des zu zählenden Makronährstoffs. In der Low-carb-Gruppe stand 1 Punkt für $1 \mathrm{~g}$ Kohlenhydrate. In den beiden Low-fat-Gruppen entsprach 1 Punkt $1 \mathrm{~g}$ 
Fett. Zusätzlich gab es in der Gruppe mit reduzierter glykämischer Last anders farbig gekennzeichnete Punkte für Kohlenhydrate mit hoher glykämischer Last. Dabei entsprach 1 Punkt 5 g Kohlenhydraten mit hoher glykämischer Last.

Zu Beginn der Ernährungsintervention erhielt jede Teilnehmerin ein kleines Heft mit einer Auswahl von ca. 700 typischen Nahrungsmitteln mit entsprechenden Grammangaben für übliche Portionsgrößen. Für jede Diätgruppe waren darin die Punktwerte entweder für Kohlenhydrate, Fett oder Fett und Kohlenhydrate mit hoher glykämischer Last aufgelistet. Diese Maßnahme sollte die praktische Durchführung der Diät vereinfachen. Die Nährwertangaben und Portionsgrößen sind der Lebensmitteltabelle „Kalorien mundgerecht“ entnommen (Nestlé Deutschland AG 2003), die auf der in Deutschland üblichen Lebensmittelpräferenz basiert und regelmäßig aktualisiert wird.

Auf Karten in DIN A6-Größe wurde die diätetisch vorgegebene Menge des jeweiligen Makronährstoffs in Form dieser Punkte aufgedruckt. Jede Karte enthielt die Punkte für eine Woche. Entsprechend der verzehrten Menge konnte die Zahl an Punkten abgehakt werden. Das ermöglichte die Selbstkontrolle der Probandinnen in Form einer flexiblen Kontrolle innerhalb einer Woche: Die der Diätphase entsprechende Mengenangabe brauchte nicht für jeden einzelnen Tag, sondern über den Zeitraum einer Woche eingehalten werden. Die Karten wurden bei den wöchentlichen Terminen in der Ernährungspsychologischen Forschungsstelle eingesammelt. Das praktische Taschenformat der Karten und des Hefts erleichterte die Mitnahme in Restaurants und für unterwegs.

\subsubsection{Diätetische Interventionen}

Für jede Diätgruppe galt dieselbe zeitliche Einteilung in 3 Phasen mit gestaffelten Vorgaben der erlaubten Mengen an Lebensmitteln beziehungsweise Punkten. In der ersten Stufe, der Eingangsphase, wurde eine stärkere Beschränkung der Menge vorgegeben, um die anfänglich hohe Motivation der Teilnehmerinnen zu nutzen und sie mit einer schnell sichtbaren Gewichtsreduktion zu belohnen. Diese Phase dauerte 4 Wochen. Die 2. Phase von der 5. bis zur 26. Woche sollte der langsamen und stetigen Gewichtsabnahme dienen. Die letzte Phase von der 27. bis zur 52. Woche sollte eine Adaptation an einen alltagskompatiblen Ernährungsstil ermöglichen. Die erreichte Gewichtsreduktion sollte erhalten, eventuell noch eine leichte weitere Gewichtsabnahme realisiert werden. Die zunächst wöchentlichen Wiegetermine wurden in dieser Phase auf monatliche Termine reduziert. 


\subsubsection{Low carb (LC)}

Die anfängliche Kohlenhydratbeschränkung wurde auf 30 g/ Tag festgelegt. In der 2. Phase wurde der Verzehr von 60 g Kohlenhydraten/ Tag erlaubt. Die letzte Diätphase zur Gewichtsstabilisierung erlaubte den Verzehr von 90 g Kohlenhydraten/ Tag. Damit fallen alle 3 Phasen der Low-carb-Diät unter die Definition einer streng kohlenhydratarmen Diät. Proteine und Fett konnten während der gesamten Interventionsphase in unbeschränktem Maß gegessen werden.

\subsubsection{Low fat $(\mathrm{LF})$}

Für die fettarme Diätvariante wurde zunächst eine Beschränkung der Fettzufuhr auf maximal $30 \mathrm{~g} /$ Tag gewählt. Nach 4 Wochen waren $45 \mathrm{~g}$ und in der letzten Phase $60 \mathrm{~g}$ Fett/ Tag erlaubt. Eine fettarme Diät enthält definitionsgemäß maximal 30\% der Energieaufnahme in Form von Fetten. $60 \mathrm{~g}$ Fett entsprechen $540 \mathrm{kcal}$ ( $1 \mathrm{~g}$ Fett entspricht $9 \mathrm{kcal})$. Das sind knapp 30\% der Energiemenge bei einer Ernährung mit 1800 kcal/ Tag. Bei normalgroßen, erwachsenen Frauen mit leichter bis mäßiger körperlicher Belastung entspricht das ungefähr einer realistischen Kost. Proteine und Kohlenhydrate durften in dieser Diätgruppe in freier Menge verzehrt werden.

\subsubsection{Low fat with reduced glycemic load (LFRGL)}

Bei dieser diätetischen Variante wurden die Vorgaben der Low-fat-Diät bezüglich der Fettbeschränkung übernommen und zusätzlich der Verzehr von Nahrungsmitteln mit hoher glykämischer Last beschränkt. Pro Tag war der Verzehr von 50 g Kohlenhydraten mit hoher glykämischer Last erlaubt. Die Einschränkung der besonders hoch gykämisch wirksamen Nahrungsmittel wurde über den gesamten Programmverlauf konstant auf maximal 50 g gehalten, um die Ernährung dieser Gruppe nicht noch stärker zu reglementieren.

\subsection{Methoden}

\subsubsection{Wiegen}

Das Wiegen wurde mit leichter Tagesbekleidung und ohne Schuhe durchgeführt. Als Waage wurde eine geeichte digitale Waage der Firma Seca, Modell 701, benutzt. Die drei für diese Untersuchung entscheidenden Gewichtsmessungen zu den Zeitpunkten T0, T1 und T2 der einzelnen Teilnehmerinnen wurden jeweils nüchtern durchgeführt. Die anderen wöchentlichen Wiegetermine konnten zu unterschiedlichen Tageszeitpunkten in nicht nüchternem Zustand erfolgen. 


\subsubsection{Körperlängenmessung}

Für die Messung der Körperlänge aller Probandinnen wurde eine geeichte Messlatte der Firma Seca verwendet. Alle Probandinnen wurden im Rahmen ihres 1. Blutentnahmetermins ohne Schuhe und im aufrechten Stand gemessen.

\subsubsection{Messung des Bauchumfangs}

Als Bauchumfang wurde die Linie in der Mitte zwischen dem unteren Rippenbogen und der Oberkante der Darmbeinschaufeln definiert. Die Messung wurde jeweils nüchtern durchgeführt und ohne Bekleidung an Bauch oder Hüften. Dazu diente ein für die Messung von Körperumfängen gefertigtes Maßband.

\subsubsection{Blutdruckmessung}

Der Blutdruck wurde systolisch und diastolisch nach der Methode von Riva-Rocci morgens bei den Blutentnahmeterminen im Sitzen nach vorheriger 3 minütiger Ruhe bestimmt. Die Messungen erfolgten mit einem automatischen Messgerät der Firma boso medicus, Typ CA 01.

\subsubsection{Blutuntersuchungen}

Alle Blutanalysen wurden im Labor des Universitätsklinikums Göttingen, Abteilung Klinische Chemie, durchgeführt. Das Labor liegt nur wenige Meter von der Ernährungspsychologischen Forschungsstelle entfernt, so dass die Blutproben in kurzer Zeit direkt zur Verarbeitung hinüber gegeben werden konnten. Die Proben wurden dort nach Eingang sofort analysiert.

\subsubsection{Hämatologische Untersuchung und Schilddrüsenwert}

Die Auszählung der Blutzellen erfolgte mittels Streulichtprinzip und Widerstandsmessung auf einem Cell Dyn 4000 der Firma Abbott. Der TSH-Wert wurde nur einmal vor Beginn der Diätintervention zum Ausschluss einer Schilddrüsenfehlfunktion nach der ECLIAMethode mit dem Gerät Elecsys 2010 (Roche) analysiert.

\subsubsection{Harnsäure und Leberwerte}

Das Analysegerät Modular (Roche) diente zur Bestimmung von Harnsäure, ALAT, ASAT, GGT und AP. Die Harnsäure wurde nach der ALDH-Methode mit Reagenzien von Rolf Greiner BioChemica gemessen. Mit dem modifizierten optimierten UV-Test der International Federation of Clinical Chemistry and Laboratory Medicine (IFCC) wurden ALAT be- 
ziehungsweise ASAT nach der Behandlung mit Reagenzien von Rolf Greiner BioChemica beziehungsweise DiaSys bestimmt. Die GGT und die AP wurden durch enzymatischen Farbtest unter Verwendung von Roche-Reagenzien ermittelt.

\subsubsection{Glucose}

Zur Bestimmung der Serumglucose diente die Hexokinase-Methode mit einem Modular-System (Roche) unter Verwendung von Roche-Reagenzien.

\subsubsection{Homocystein}

Die Homocystein-Messung erfolgte auf einem AxSym-Analyser (Abbott) mittels Immunoassay (FPIA) unter Verwendung von Abbott-Reagenzien.

\subsubsection{Hochsensitives C-reaktive Protein (hsCRP)}

Die hsCRP-Bestimmung wurde mit latexverstärktem Immunoassay auf einem Integra 800 (Roche) ebenfalls unter Verwendung von Roche-Reagenzien durchgeführt.

\subsubsection{Methylen-Tetrahydrofolat-Reduktase-Genotypisierung}

Durch die Echtzeit-Fluoreszenz-PCR-Methode (real-time fluorescence PCR) mit Hilfe des LightCycler (Roche) konnte die Genotypisierung der MTHFR vorgenommen werden (von Ahsen et al. 1999).

\subsubsection{Datenerfassung und Statistik}

\subsubsection{MediTouch-Programm}

MediTouch ist ein computergestütztes Programm zur Erfassung und Auswertung von Lebensmittelverzehrdaten (Faustin 2004). Mit Hilfe dieses interaktiven Computerprogramms wurden vor und nach 6 Monaten Diätintervention die Ernährungsgewohnheiten aller Teilnehmerinnen erhoben. Aus den angegebenen Daten konnten die Aufnahmemengen von Kalorien, Makronährstoffen und einzelnen Mikronährstoffen wie Folsäure, Vitamin B 6 und B 12 berechnet werden.

\subsubsection{Datenerfassung und statistische Auswertung}

Zur Aufbereitung und Berechnung der deskriptiven Statistik aus den erhobenen Daten wurde das Programm SPSS Version 12.0 von Microsoft verwendet. Zunächst wurden die Mittelwerte, Mediane, Standardabweichungen, Maxima und Minima aller Daten für die einzelnen Diätgruppen bestimmt. Dann wurde die Normalverteilung der einzelnen Datenmengen grafisch überprüft. Die anschließend durchgeführten Mittelwertvergleiche wurden mit einer 
zweifaktoriellen Varianzanalyse (ANOVA) durchgeführt. Dabei setzten wir für normal verteilte Datenmengen ein parametrisches und für nicht normal verteilte ein nicht-parametrisches Testverfahren ein. Differenzen wurden von uns als signifikant angesehen, wenn die Irrtumswahrscheinlichkeit $\mathrm{p}$ kleiner als $5 \%$ war, also bei $\mathrm{p}<0,05$. Als Software für die Berechnung der ANOVA wurde die Software SAS Version 9.1 verwendet.

Die Korrelationsanalysen und die grafische Darstellung der Abhängigkeiten zwischen zwei oder drei Parametern inklusive Einzeichnen der linearen Regressionsgeraden erfolgten wiederum mit der Software SPSS. Bei normal verteilten Datenmengen wurde der Korrelationskoeffizient nach Pearson berechnet und bei nicht normalverteilten Daten der Rangkorrelationskoeffizient nach Spearman. Für alle Koeffizienten wurde ebenfalls die Irrtumswahrscheinlichkeit $\mathrm{p}$ angegeben. 


\section{Ergebnisse}

\subsection{Wertung, Randomisierung und Schichtung der Probandinnen}

\subsubsection{Wertung der Probandinnen}

Nach der Auswahl der Probandinnen anhand der genannten Kriterien wurden 160 der interessierten Frauen in das Programm aufgenommen. Wie aus anderen Diätuntersuchungen bekannt, brachen im Verlauf der 6 Monate zahlreiche Frauen das Diätprogramm vorzeitig ab. Von den 53 Teilnehmerinnen der Gruppe LC beendeten 24 Teilnehmerinnen ( 45,3\%) das Programm. In der Gruppe LF waren es von anfänglich 54 Frauen 19 ( 35,2 \%) und am wenigsten mit 16 von 53 Frauen ( 30,2 \%) in der Gruppe LFRGL, die ausschieden. Insgesamt hörten 59 von 160 Teilnehmerinnen vorzeitig auf. Das entspricht einer Abbrecherquote von 36,9\%. Für die aktuelle Per-protocol-Auswertung wurden nur Daten von Teilnehmerinnen aufgenommen, die zu allen 3 Blutabnahmeterminen - vorher, nach 4 Wochen und nach 6 Monaten Diät - anwesend waren und in den zwischenzeitlich erfolgten wöchentlichen Wiegeterminen nur geringe Fehltermine aufwiesen. Daher fielen alle Abbrecherinnen aus der Wertung heraus.

Zusätzlich mussten wir 3 Teilnehmerinnen aus der Gruppe LC, 7 aus LF und 5 aus LFRGL aufgrund von erhöhten hsCRP-Werten von der Wertung ausnehmen. Werte größer als $8 \mathrm{mg} / \mathrm{l}$ werden im Labor des Uniklinikums Göttingen nicht weiter differenziert. Ein wichtiges Argument für einen Cut-off bei $8 \mathrm{mg} / \mathrm{l}$ ist die Tatsache, dass nach bisherigen Erfahrungen bei Übersteigen dieser Grenze die Wahrscheinlichkeit einer hsCRP-Erhöhung aus anderen Ursachen als durch eine leichte chronische Gefäßentzündung ansteigt. So wird das hsCRP zur Einschätzung des kardiovaskulären Risikos unbrauchbar. Daher setzten wir bei einmaligem Messen eines Wertes von größer als $8 \mathrm{mg} / \mathrm{l}$ ein Ausschlusskriterium für die Auswertung an. Es wurden entsprechend der Per-protocol-Vorgabe alle Daten der jeweiligen Probandin herausgenommen. Darüber hinaus mussten 2 Teilnehmerinnen, je eine aus LF und LFRGL, wegen massiv erhöhten Triglyceridwerten in der T2-Blutuntersuchung ausgeschlossen werden, da die Werte am ehesten durch deutliche Compliance-Mängel oder fehlende Nüchternheit zu erklären sind.

Die vorliegende Untersuchung bedient sich ausschließlich kompletter Datensätzen nach Protokoll des Programms, mit dem zusätzlichen Ausschlusskriterium von hsCRP-Werte >8 
$\mathrm{mg} / \mathrm{l}$ und entspricht damit einer Per-protocol-Analyse. Nach diesen Kriterien konnten in die Auswertung 84 der zuvor 160 Teilnehmerinnen einbezogen werden, dass sind 52,5\% der anfänglichen Teilnehmerinnenzahl.

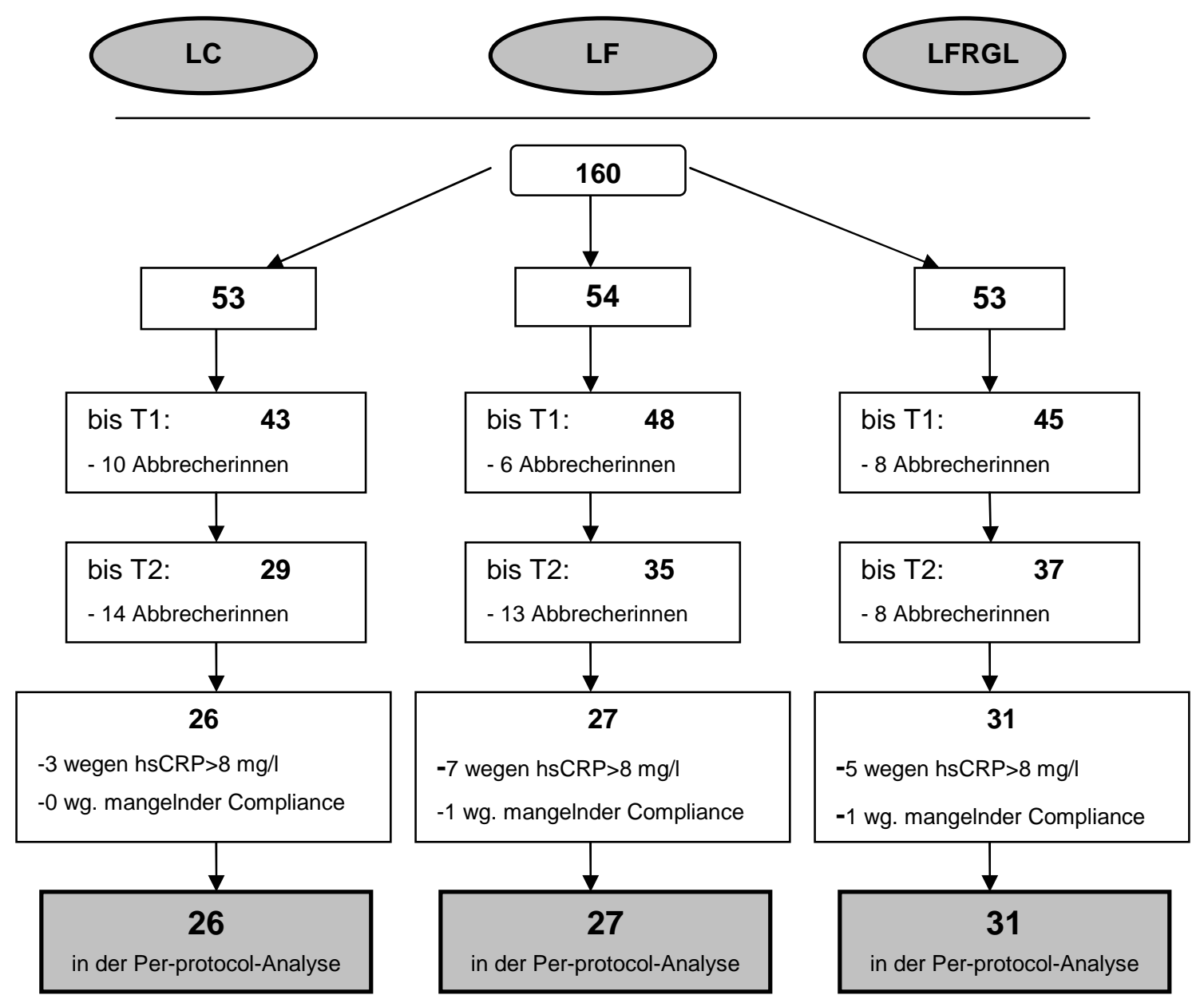

Abbildung 3: Abbrecherquote und Ausschlüsse aus der Per-protocol-Analyse

\subsubsection{Altersstruktur der Gruppen}

Durch die Abbrecherinnen und die aus der Wertung herausgenommenen Teilnehmerinnen ergibt sich in dieser Untersuchung für alle verbleibenden Frauen ein Durchschnittsalter von 51 Jahren (SD 10; Min. 28; Max. 68). Das mittlere Alter in der Gruppe LC liegt bei 52,4 Jahren (SD 10,3; Min. 33; Max. 68), in der Gruppe LF bei 49,6 (SD 11,4; Min. 28; Max. 68) und in der Gruppe LFRGL bei 51,1 Jahren (SD 8,6; Min. 36; Max. 67). Obwohl es sich in den Gruppen durch das Ausscheiden von Probandinnen nach der Randomisierung und Schichtung nach Alter und BMI leicht verändert hat, sind die Unterschiede zwischen den Gruppen mit $\mathrm{p}=0,602$ nicht signifikant. 
Altersstruktur der Gruppen zum Zeitpunkt T0

\begin{tabular}{|l|c|c|c|c|c|c|}
\hline Gruppe & N & Mittel & Median & SD & Min. & Max. \\
\hline Alle & 84 & 51 & 50 & 10 & 28 & 68 \\
LC & 26 & 52,4 & 51,5 & 10,3 & 33 & 68 \\
LF & 27 & 49,6 & 52 & 11,4 & 28 & 68 \\
LFRGL & 31 & 51,1 & 49 & 8,6 & 36 & 67 \\
\hline
\end{tabular}

Tabelle 1: Altersstruktur der Gruppen zum Zeitpunkt T0

\subsubsection{Mittlerer BMI der Gruppen zum Zeitpunkt T0}

Das zweite Schichtungskriterium nach der Randomisierung war der initiale BMI. Auch hier ergaben sich durch das nachträgliche Ausscheiden von Teilnehmerinnen geringe Verschiebungen ohne statistische Signifikanz ( $\mathrm{p}=0,593)$. Der mittlere BMI lag vor Beginn der Diätintervention für alle Frauen zusammen bei 30,8 kg/m² (SD 3,4; Min. 25; Max. 40,7). In der Gruppe LC fand sich ein mittlerer BMI von 31,4 kg/m² (SD 2,8; Min. 25,8; Max. 35,9), in der Gruppe LF von 30,5 kg/m² (SD 3,9; Min. 24,6; Max. 40,7) und in der Gruppe LFRGL von 30,7 kg/m² (SD 3,4; Min. 25; Max. 37,3).

\section{Anfänglicher BMI}

\begin{tabular}{|l|c|c|c|c|c|c|}
\hline Gruppe & N & Mittel & Median & SD & Min. & Max. \\
\hline Alle & 84 & 30,8 & 30,85 & 3,4 & 25 & 35 \\
LC & 26 & 31,4 & 31,8 & 2,8 & 25,8 & 35,9 \\
LF & 27 & 30,5 & 29,8 & 3,9 & 24,6 & 40,7 \\
LFRGL & 31 & 30,7 & 30,3 & 3,4 & 25 & 37,3 \\
\hline
\end{tabular}

Tabelle 2: BMI der Gruppen zum Zeitpunkt T0

\subsubsection{Raucherstatus, Kaffeekonsum und Vitaminsupplementation}

Unter den Probandinnen fanden sich nur wenige aktive oder ehemalige Raucherinnen. 11 der 84 Frauen gaben einen aktiven Nikotinkonsum an und 4 waren ehemalige Raucherinnen. Als Raucherin klassifiziert sind 3 von 26 in Gruppe LC, 5 von 27 in Gruppe LF und 6 von 31 in Gruppe LFRGL. Für den Kaffeekonsum von 6 und mehr Tassen Kaffee täglich gilt ähnliches: Je 1 Teilnehmerin aus jeder Gruppe trinkt viel Kaffee. Bei der täglichen Vitaminsupplementation finden sich leichte Unterschiede zwischen den Gruppen. 2 Frauen aus Gruppe LC, 4 aus LF und 9 aus LFRGL gaben vor und während der ersten 4 Wochen der Diätintervention die Einnahme von zusätzlichen Vitaminen an. Die Menge und Art der eingenommenen Vitaminpräparate wurde nicht ermittelt. 


\subsection{Darstellung der Messergebnisse und der Signifikanztests}

Alle Messergebnisse, die der Messungen und der Laboranalysen werden mit Mittelwert, Median, Standardabweichung, Minimum und Maximum tabellarisch und die Hauptparameter auch grafisch als Boxplots dargestellt. Die Boxplots setzen sich zusammen aus einer farbigen Box, deren untere Begrenzung vom 1. Quartil (25. Perzentil) und deren obere Begrenzung vom 3. Quartil (75. Perzentil) gebildet werden. Eine kräftige schwarze Linie im mittleren Bereich der Boxplots stellt den Median dar. Zusätzlich sind Minimum und Maximum als Tförmig endende Linien eingezeichnet, es sei denn die Werte werden durch Ausreißer gebildet. Als Ausreißer sind Werte definiert, die mehr als eineinhalb beziehungsweise drei Boxenlängen außerhalb liegen. Sie werden als Kreise beziehungsweise Sternchen abgebildet.

Die Differenzen der Messergebnisse sind jeweils als Ergebnisse der Subtraktionen der Messzeitpunkte T1-T0, T2-T1 und T2-T0 noch extra tabellarisch aufgeführt, ebenfalls mit Mittelwert, Median, Standardabweichung, Minimum und Maximum. Ein negatives Ergebnis spiegelt eine Abnahme und ein positives Ergebnis eine Zunahme des Wertes gegenüber dem vorherigen Zeitpunkt wider.

Die Signifikanzüberprüfungen wurden, wie oben erwähnt, mit einer parametrischen zweifaktoriellen Varianzanalyse (ANOVA) bei normalverteilten Datenmengen durchgeführt und mit einer nichtparametrischen zweifaktoriellen ANOVA bei nicht normalverteilten Datenmengen. Der angegebene p-Wert sagt aus, ob eventuelle Unterschiede signifikant sind. Bei zugrunde gelegtem zweiseitigem Signifikanzniveau $\alpha=0,05$ sind die Differenzen bei einem $p$ Wert $<0,05$ signifikant. Der $\alpha$-Fehler beträgt maximal 5\%. Angegeben wird jeweils der pWert für die Signifikanz der Gruppenunterschiede zu den einzelnen Zeitpunkten, für die Veränderung des Parameters über die Zeit in den einzelnen Gruppen und für die Unterschiede der zeitlichen Veränderungen zwischen den Gruppen. Gibt es für den letzteren p-Wert ein signifikantes Ergebnis, unterscheiden sich also die Zeitverläufe signifikant zwischen den Gruppen, dann werden für jede Gruppe einzelne p-Werte bezüglich des Zeitverlaufs angegeben. In diesem Fall ist die Überprüfung auf signifikante Gruppenunterschiede hinfällig, da sie über die Zeit definitiv bestehen. Der p-Wert für die Gruppen entfällt in diesem Fall. 


\subsection{Körperparameter}

\subsubsection{Körpergewicht, BMI und Bauchumfang}

\subsubsection{Ergebnisse der Messungen}

Für Körpergewicht, BMI und Bauchumfang in den 3 Untersuchungsgruppen ergaben sich die folgenden Ergebnisse zu den 3 Messzeitpunkten T0, T1 und T2:

\section{Körpergewicht in kg}

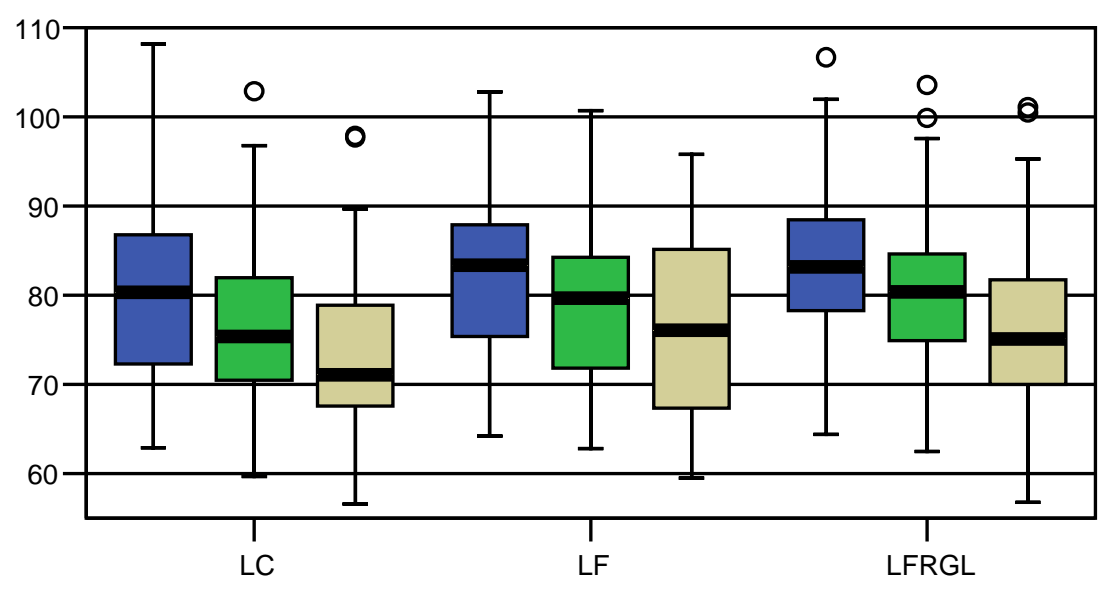

\begin{tabular}{|l|ccc|ccc|cccc|}
\hline & \multicolumn{2}{|l}{ Gruppe LC } & \multicolumn{3}{|c|}{ Gruppe LF } & & \multicolumn{3}{c|}{ Gruppe LFRGL } \\
Zeitpunkt & T0 & T1 & T2 & T0 & T1 & T2 & T0 & T1 & T2 \\
\hline Mittel & 81,4 & 77,7 & 73,3 & 83 & 80 & 76,7 & 84,1 & 80,9 & 77,2 \\
Median & 80,4 & 75,4 & 71,1 & 83,4 & 79,7 & 76,1 & 83,2 & 80,4 & 75,1 \\
SD & 11,2 & 10,6 & 10,6 & 10,8 & 10,3 & 10,8 & 9,8 & 9,9 & 11 \\
Min. & 62,9 & 59,7 & 56,6 & 64,2 & 62,8 & 59,5 & 64,4 & 62,5 & 56,8 \\
Max. & 108,2 & 102,9 & 97,9 & 102,8 & 100,7 & 95,8 & 106,7 & 103,6 & 101,1 \\
\hline
\end{tabular}

\begin{tabular}{|c|c|c|c|c|c|c|c|c|c|}
\hline \multirow[b]{2}{*}{ Differenz } & \multicolumn{3}{|c|}{ Gruppe LC } & \multicolumn{3}{|c|}{ Gruppe LF } & \multicolumn{3}{|c|}{ Gruppe LFRGL } \\
\hline & T1-T0 & T2-T1 & T2-T0 & T1-T0 & $\mathrm{T} 2-\mathrm{T} 1$ & T2-T0 & T1-T0 & T2-T1 & T2-T0 \\
\hline Mittel & $-3,7$ & $-4,4$ & $-8,1$ & $-3,0$ & $-3,3$ & $-6,3$ & $-3,2$ & $-3,6$ & $-6,8$ \\
\hline Median & $-3,6$ & $-4,1$ & $-8,0$ & $-2,8$ & $-2,8$ & $-6,1$ & $-3,3$ & $-3,6$ & $-6,9$ \\
\hline SD & 1,7 & 3,1 & 4,0 & 1,4 & 3,2 & 3,9 & 1,3 & 2,7 & 3,5 \\
\hline Min. & $-7,5$ & $-11,1$ & $-17,0$ & $-5,7$ & $-10,9$ & $-15,4$ & $-5,5$ & $-9,3$ & $-13,8$ \\
\hline Max. & $-0,7$ & 1,1 & $-2,1$ & $-0,8$ & 2,6 & 0,0 & 0,3 & 1,2 & 0,1 \\
\hline \multicolumn{4}{|c|}{ Analyse der Gruppen } & \multicolumn{2}{|c|}{$p=0,4868$} & & & & \\
\hline \multicolumn{4}{|c|}{ Analyse der Zeitdifferenz } & \multicolumn{2}{|c|}{$p<0,0001$} & & & & \\
\hline \multicolumn{4}{|c|}{$\begin{array}{l}\text { Analyse der Wechselwirkung Gruppe } \\
\text { und Zeit }\end{array}$} & \multicolumn{2}{|c|}{$p=0,2465$} & & & & \\
\hline
\end{tabular}

Tabelle 3: Gewichtsabnahme in $\mathrm{kg}$ 
BMI in $\mathrm{kg} / \mathrm{m}^{2}$

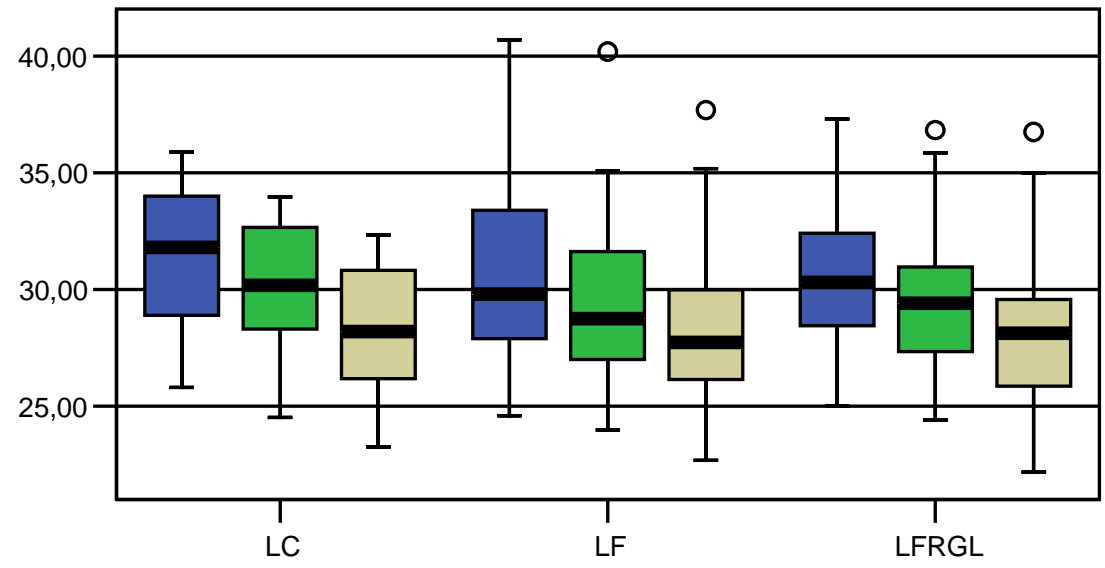

BMI TO in $\mathrm{kg} / \mathrm{m}^{2}$

$\square$ BMI T1

$\square$ BMI T2

LFRGL

\begin{tabular}{|l|ccc|ccc|cccc|}
\hline & \multicolumn{2}{|l}{ Gruppe LC } & \multicolumn{3}{|c|}{ Gruppe LF } & \multicolumn{3}{c|}{ Gruppe LFRGL } \\
Zeitpunkt & T0 & T1 & T2 & T0 & T1 & T2 & T0 & T1 & T2 \\
\hline Mittel & 31,4 & 30,1 & 28,4 & 30,5 & 29,5 & 28,3 & 30,7 & 29,8 & 28,4 \\
Median & 31,8 & 30,2 & 28,2 & 29,8 & 28,7 & 27,7 & 30,3 & 29,4 & 28,1 \\
SD & 2,8 & 2,7 & 2,7 & 3,9 & 3,8 & 3,8 & 3,4 & 3,3 & 3,6 \\
Min. & 25,8 & 24,5 & 23,3 & 24,6 & 24 & 22,7 & 25 & 24,4 & 22,2 \\
Max. & 35,9 & 34 & 32,3 & 40,7 & 40,2 & 37,7 & 37,3 & 36,8 & 36,7 \\
\hline
\end{tabular}

\begin{tabular}{|c|c|c|c|c|c|c|c|c|c|}
\hline \multirow[b]{2}{*}{ Differenz } & \multicolumn{3}{|c|}{ Gruppe LC } & \multicolumn{3}{|c|}{ Gruppe LF } & \multicolumn{3}{|c|}{ Gruppe LFRGL } \\
\hline & T1-T0 & $\mathrm{T} 2-\mathrm{T} 1$ & T2-T0 & T1-T0 & $\mathrm{T} 2-\mathrm{T} 1$ & T2-T0 & T1-T0 & T2-T1 & T2-T0 \\
\hline Mittel & $-1,3$ & $-1,7$ & $-3,0$ & $-1,0$ & $-1,3$ & $-2,2$ & $-0,9$ & $-1,4$ & $-2,3$ \\
\hline Median & $-1,3$ & $-1,6$ & $-2,9$ & $-0,9$ & $-1,0$ & $-2,0$ & $-0,9$ & $-1,3$ & $-2,2$ \\
\hline SD & 0,8 & 1,2 & 1,5 & 0,6 & 1,2 & 1,7 & 0,7 & 1,1 & 1,5 \\
\hline Min. & $-3,9$ & $-5,0$ & $-6,0$ & $-2,2$ & $-4,2$ & $-6,3$ & $-2,4$ & $-3,8$ & $-5,8$ \\
\hline Max. & $-0,2$ & 0,4 & $-0,6$ & 0,3 & 0,9 & 0,5 & 2,0 & 0,4 & 0,9 \\
\hline \multicolumn{4}{|c|}{ schiede } & \multicolumn{2}{|c|}{$p=0,8153$} & & & & \\
\hline \multicolumn{4}{|c|}{ Analyse der Zeitdifferenz } & \multicolumn{2}{|c|}{$p<0,0001$} & & & & \\
\hline \multirow{2}{*}{\multicolumn{4}{|c|}{$\begin{array}{l}\text { Analyse der Wechselwirkung Gruppe } \\
\text { und Zeit }\end{array}$}} & \multirow{2}{*}{\multicolumn{2}{|c|}{$p=0,1432$}} & & & & \\
\hline & & & & & & & & & \\
\hline
\end{tabular}

Tabelle 4: BMI-Differenz 


\section{Bauchumfang in cm}

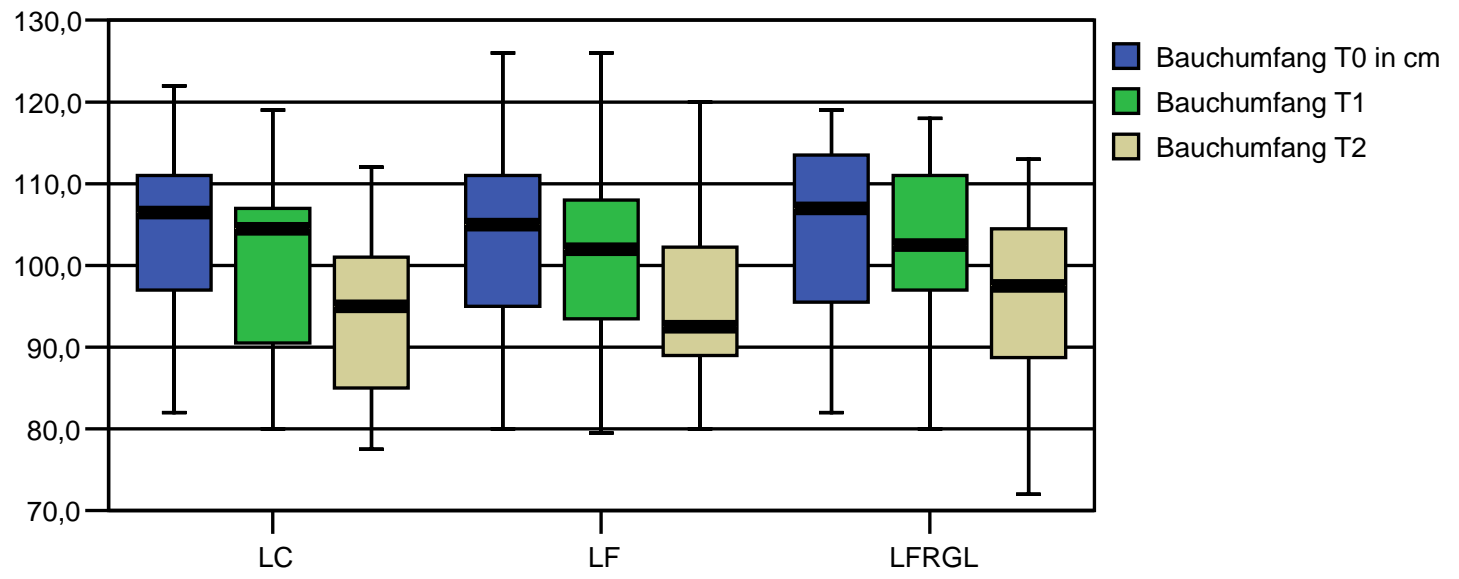

\begin{tabular}{|l|ccc|ccc|ccc|}
\hline & \multicolumn{3}{|l}{ Gruppe LC } & \multicolumn{3}{|c|}{ Gruppe LF } & \multicolumn{3}{c|}{ Gruppe LFRGL } \\
Zeitpunkt & T0 & T1 & T2 & T0 & T1 & T2 & T0 & T1 & T2 \\
\hline Mittel & 103,7 & 100,6 & 93,9 & 103,8 & 101,9 & 95,8 & 104,9 & 102,5 & 97,0 \\
Median & 106,5 & 104,5 & 95 & 105 & 102 & 92,5 & 107 & 102,5 & 97,5 \\
SD & 11,7 & 11,2 & 9,1 & 11,3 & 10,9 & 10,8 & 10,4 & 10,1 & 11 \\
Min. & 82 & 80 & 77,5 & 80 & 79,5 & 80 & 82 & 80 & 72 \\
Max. & 122 & 119 & 112 & 126 & 126 & 120 & 119 & 118 & 113 \\
\hline
\end{tabular}

\begin{tabular}{|c|c|c|c|c|c|c|c|c|c|}
\hline \multirow[b]{2}{*}{ Differenz } & \multicolumn{3}{|c|}{ Gruppe LC } & \multicolumn{3}{|c|}{ Gruppe LF } & \multicolumn{3}{|c|}{ Gruppe LFRGL } \\
\hline & T1-T0 & T2-T1 & T2-Т0 & T1-T0 & T2-T1 & T2-T0 & T1-T0 & T2-T1 & T2-T0 \\
\hline Mittel & $-3,1$ & $-6,7$ & $-9,7$ & $-1,9$ & $-6,1$ & -8 & $-2,4$ & $-5,5$ & $-7,9$ \\
\hline Median & -3 & $-5,5$ & $-9,3$ & -2 & -5 & $-6,5$ & -2 & $-4,5$ & -6 \\
\hline SD & 3,3 & 4,6 & 5,8 & 3,7 & 6,4 & 6,6 & 2,5 & 4,6 & 5,4 \\
\hline Min. & -12 & -20 & -25 & -10 & -19 & -23 & -8 & -14 & -19 \\
\hline Max. & 5 & -1 & -1 & 12 & 1 & 0,5 & 2 & 2 & 0 \\
\hline \multicolumn{4}{|c|}{ Analyse der Gruppenunterschiede } & \multicolumn{2}{|c|}{$p=0,7541$} & & & & \\
\hline \multicolumn{4}{|c|}{ Analyse der Zeitdifferenz } & \multicolumn{2}{|c|}{$p<0,0001$} & & & & \\
\hline \multicolumn{4}{|c|}{$\begin{array}{l}\text { Analyse der Wechselwirkung Gruppe } \\
\text { und Zeit }\end{array}$} & \multicolumn{2}{|c|}{$p=0,5747$} & & & & \\
\hline
\end{tabular}

Tabelle 5: Änderung des Bauchumfangs

\subsubsection{Vergleich der Messungen}

In allen drei Gruppen ist eine signifikante Gewichtsabnahme jeweils bis T1 und bis T2 erfolgt. Zwar ist in der Gruppe LC durchschnittlich mit 8,1 kg (9,9\% des Ausgangsgewichts) eine größere Abnahme nach 6 Monaten sichtbar als in den Gruppen LF mit 6,3 kg (7,6\%) und LFRGL mit 6,8 kg (8,3\%). Diese Unterschiede zwischen den Gruppen sind allerdings nicht signifikant. Das gleiche gilt für die Gewichtsabnahme nach 4 Wochen (T1). Es besteht zu keinem Zeitpunkt ein signifikanter Unterschied zwischen den Gruppen bezüglich der mittleren Körpergewichte oder im Zeitverlauf der Gewichtsabnahme. Dies ergibt die parametrische 
zweifaktorielle Varianzanalyse (ANOVA) der Ergebnisse der Gewichtsabnahme über die Zeit und zwischen den 3 Diätgruppen bei normalverteilten Gewichtsdaten.

Wenn man das Erreichen einer Gewichtsreduktion von 5 bzw. 10 Prozent des Ausgangsgewicht als Erfolgskriterium formuliert, ergeben sich nach 6 Monaten (T2) folgende Ergebnisse: 21 von 26 Teilnehmerinnen (80\%) aus der Gruppe LC haben mehr als 5\% ihres Ausgangsgewichts verloren. In den Gruppen LF sind es 19 von 27 Teilnehmerinnen (70\%) und in LFRGL 25 von 31 der Teilnehmerinnen (ebenfalls ca. 80\%). Eine 10-prozentige Gewichtsreduktion haben in LC 11 von 26 der Teilnehmerinnen (42\%) erreicht, in LF 7 von 27 (26\%) und in LFRGL 11 von 31 (35\%). Dieses Ergebnis spiegelt die in der Statistik nicht signifikante Tendenz zur größeren Gewichtsabnahme in der Gruppe LC gegenüber LFRGL und letztlich LF mit der geringsten Abnahme recht gut wieder.

Im Vergleich zur Per-protocol-Analyse zeigen die Ergebnisse der Intention-to-treatAnalyse eine etwas geringere Gewichtsabnahme in allen Gruppen. Wendet man die Methode Last-observation-carried-forward zum Ersatz der fehlenden Gewichtsdaten an, bleibt die beschriebene Tendenz zum stärksten Gewichtsverlust in LC, gefolgt von LFRGL und LF mit der geringsten Gewichtsreduktion bestehen:

\section{Gewichtsentwicklung in der Intention-to-treat-Analyse}

\begin{tabular}{|l|cc|c|cc|c|cc|c|}
\hline & \multicolumn{3}{|c|}{ Gruppe LC (N=43) } & \multicolumn{3}{c|}{ Gruppe LF (N=48) } & \multicolumn{3}{c|}{ Gruppe LFRGL (N=46) } \\
Zeitpunkt & T0 & T2 & T2-T0 & T0 & T2 & T2-T0 & T0 & T2 & T2-T0 \\
\hline Mittel & 81,4 & 75,2 & $-6,3$ & 84,3 & 79,1 & $-5,1$ & 82,6 & 76,6 & $-6,0$ \\
Median & 81,2 & 76,6 & $-6,1$ & 85,0 & 78,4 & $-3,7$ & 83,1 & 75,1 & $-6,3$ \\
SD & 10,0 & 10,3 & 4,6 & 9,8 & 10,2 & 3,9 & 10,4 & 10,6 & 4,3 \\
Min. & 62,9 & 56,6 & $-17,0$ & 64,2 & 59,5 & $-15,4$ & 64,4 & 56,8 & $-16,6$ \\
Max. & 108,2 & 97,9 & 0,0 & 102,8 & 99,3 & 0,10 & 106,7 & 101,1 & 1,0 \\
\hline
\end{tabular}

Tabelle 6: Intention-to-treat-Analyse der Gewichtsveränderungen

Aus den Körpergewichten in der Analyse der kompletten Daten konnten jeweils die BMI-Werte berechnet werden, mit der entsprechend etwas größeren Abnahme in der Gruppe LC mit $-3 \mathrm{~kg} / \mathrm{m}^{2}$ gegenüber $-2,2 \mathrm{~kg} / \mathrm{m}^{2}$ beziehungsweise $-2,3 \mathrm{~kg} / \mathrm{m}^{2}$ in LF beziehungsweise LFRGL. Die Varianzanalyse ergibt signifikante Änderungen über die Zeit, aber keine signifikanten Unterschiede zwischen den Gruppen.

Als weiterer Parameter liefert die Messung des Bauchumfangs Informationen über die Körperkonstitution. Die deutliche Abnahme der Bauchumfangsdifferenz entspricht ebenfalls den Gewichtsverläufen mit größter Differenz von T0 zu T2 in LC mit -9,7 cm. Die beiden 
Gruppen LF und LFRGL unterscheiden sich hierin nur minimal. Die Signifikanztestung belegt bezüglich Gewicht und BMI fehlende signifikante Unterschiede zwischen den Gruppen, aber signifikante Differenzen über die Zeit innerhalb einer Gruppe.

\subsubsection{Systolischer und diastolischer Blutdruck}

\subsubsection{Ergebnisse der Messungen}

Die Werte der systolischen und diastolischen Blutdruckwertmessungen der 3 Gruppen zu den Zeitpunkten T0, T1 und T2 werden im Folgenden ebenfalls tabellarisch und als Boxplots dargestellt:

\section{Blutdruck systolisch in $\mathbf{m m ~} \mathrm{Hg}$}

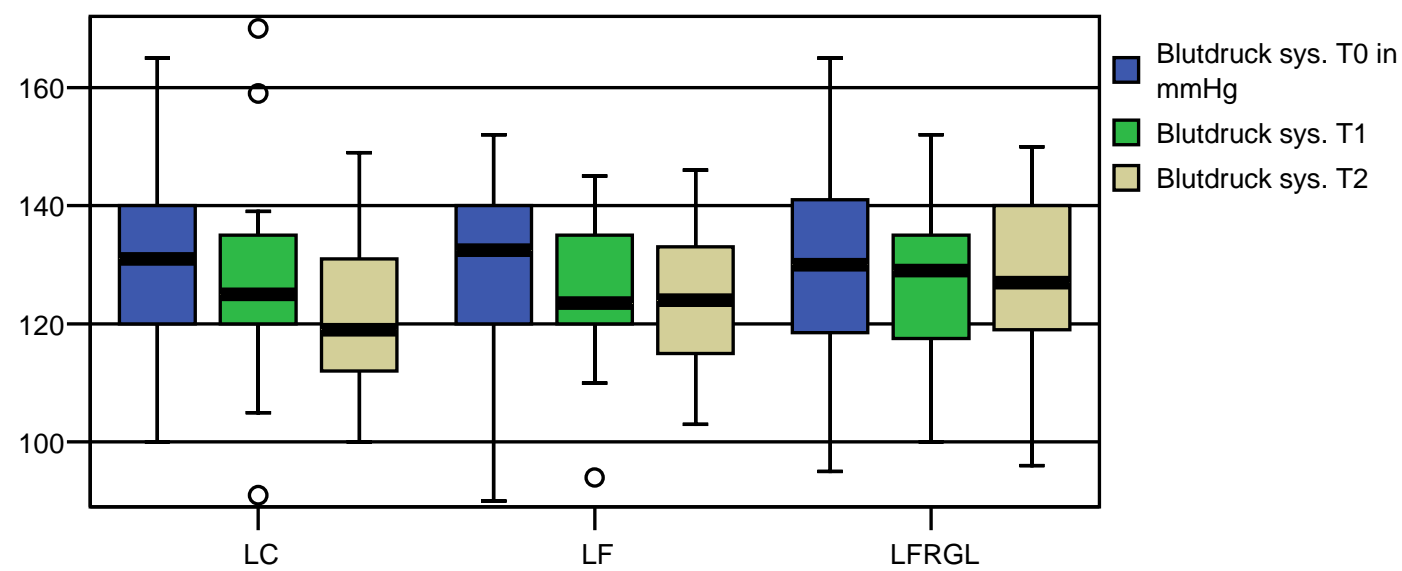

\begin{tabular}{|l|ccc|ccc|ccc|}
\hline & \multicolumn{3}{|l}{ Gruppe LC } & \multicolumn{4}{|l|}{ Gruppe LF } & \multicolumn{3}{|c|}{ Gruppe FRGL } \\
Zeitpunkt & T0 & T1 & T2 & T0 & T1 & T2 & T0 & T1 & T2 \\
\hline Mittel & 129,5 & 126,1 & 119,5 & 127,7 & 126,1 & 123,8 & 129,8 & 126,3 & 127,3 \\
Median & 131,0 & 125,0 & 119,0 & 135,0 & 124,0 & 124,0 & 130,0 & 129,0 & 127,0 \\
SD & 15,1 & 16,2 & 12,9 & 16,7 & 12,2 & 11,4 & 18,4 & 13,5 & 14,2 \\
Min. & 100,0 & 91,0 & 100,0 & 90,0 & 94,0 & 103,0 & 95,0 & 100,0 & 96,0 \\
Max. & 165,0 & 170,0 & 149,0 & 152,0 & 145,0 & 146,0 & 165,0 & 152,0 & 150,0 \\
\hline
\end{tabular}




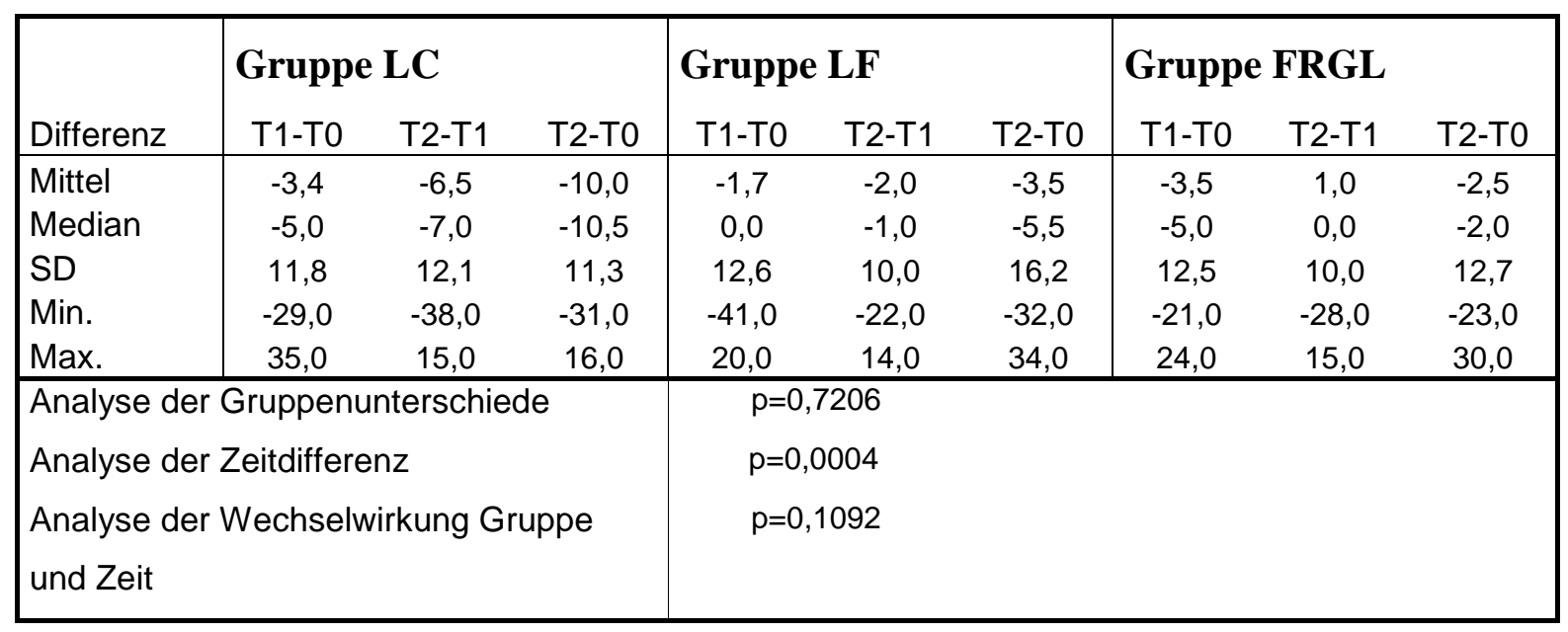

Tabelle 7: Änderung des systolischen Blutdrucks

\section{Blutdruck diastolisch in $\mathbf{~ m m ~} \mathbf{H g}$}

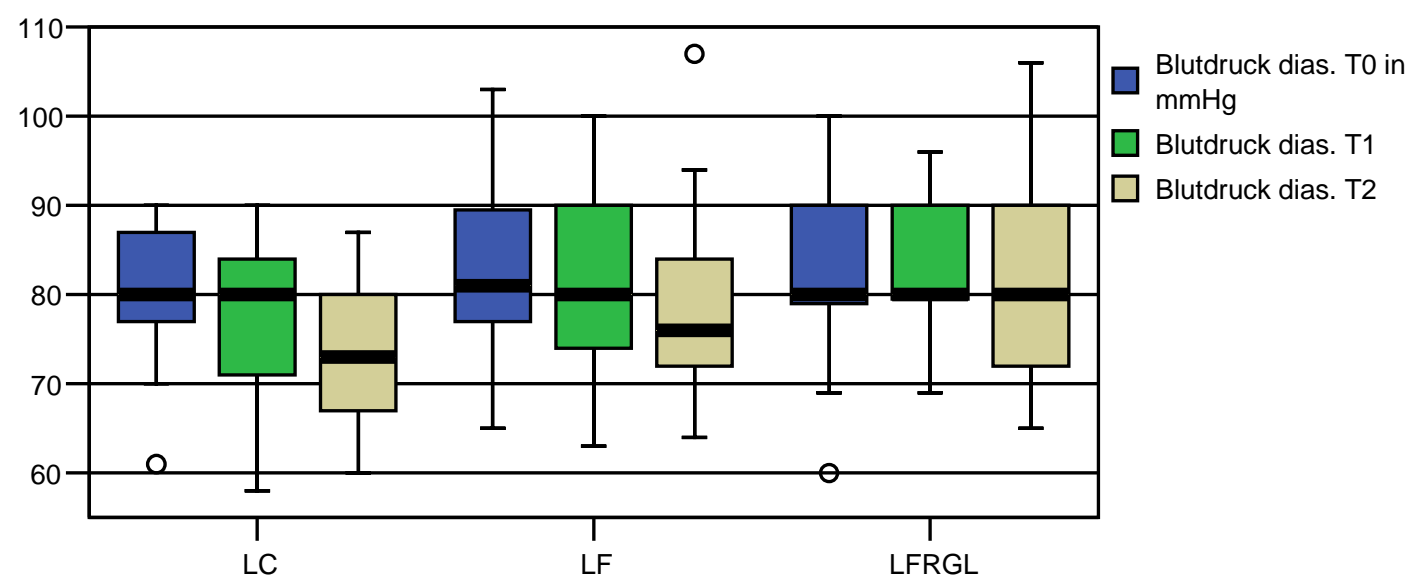

\begin{tabular}{|l|ccc|ccc|ccc|}
\hline & \multicolumn{3}{|l}{ Gruppe LC } & \multicolumn{4}{|l|}{ Gruppe LF } & \multicolumn{3}{|c|}{ Gruppe FRGL } \\
Zeitpunkt & T0 & T1 & T2 & T0 & T1 & T2 & T0 & T1 & T2 \\
\hline Mittel & 81,0 & 78,1 & 73,0 & 82,7 & 81,5 & 78,2 & 82,9 & 82,2 & 82,1 \\
Median & 80,0 & 80,0 & 73,0 & 81,0 & 80,0 & 76,0 & 80,0 & 80,0 & 80,0 \\
SD & 7,4 & 8,7 & 7,6 & 9,4 & 9,2 & 10,0 & 8,7 & 7,9 & 10,7 \\
Min. & 61,0 & 58,0 & 60,0 & 65,0 & 63,0 & 64,0 & 60,0 & 69,0 & 65,0 \\
Max. & 90,0 & 90,0 & 87,0 & 103,0 & 100,0 & 107,0 & 100,0 & 96,0 & 106,0 \\
\hline
\end{tabular}




\begin{tabular}{|c|c|c|c|c|c|c|c|c|c|}
\hline \multirow[b]{2}{*}{ Differenz } & \multicolumn{3}{|c|}{ Gruppe LC } & \multicolumn{3}{|c|}{ Gruppe LF } & \multicolumn{3}{|c|}{ Gruppe FRGL } \\
\hline & T1-T0 & $\mathrm{T} 2-\mathrm{T} 1$ & T2-T0 & T1-T0 & $\mathrm{T} 2-\mathrm{T} 1$ & T2-T0 & T1-T0 & $\mathrm{T} 2-\mathrm{T} 1$ & T2-T0 \\
\hline Mittel & $-2,8$ & $-5,1$ & $-7,9$ & $-1,2$ & $-3,3$ & $-4,5$ & $-0,7$ & $-0,1$ & $-0,8$ \\
\hline Median & $-3,0$ & $-4,0$ & $-7,0$ & 0,0 & $-3,0$ & $-6,0$ & 0,0 & 0,0 & $-2,0$ \\
\hline SD & 6,8 & 7,4 & 6,1 & 8,5 & 8,9 & 10,1 & 6,6 & 9,2 & 9,8 \\
\hline Min. & $-15,0$ & $-23,0$ & $-26,0$ & $-17,0$ & $-25,0$ & $-25,0$ & $-15,0$ & $-18,0$ & $-19,0$ \\
\hline Max. & 10,0 & 7,0 & 3,0 & 16,0 & 12,0 & 18,0 & 10,0 & 30,0 & 29,0 \\
\hline \multicolumn{4}{|c|}{ Analyse der Gruppenunterschiede } & & & & & & \\
\hline \multicolumn{4}{|c|}{ Analyse der Zeitdifferenz } & \multicolumn{6}{|c|}{$\mathrm{p}<0,0001$ (LC); $\mathrm{p}=0,0298$ (LF); $\mathrm{p}=0,6877$ (LFRGL) } \\
\hline \multicolumn{4}{|l|}{ T1-T0 } & \multicolumn{6}{|c|}{$\mathrm{p}=, 0612(\mathrm{LC}) ; \mathrm{p}=0,5222(\mathrm{LF}) ; \mathrm{p}=0,6843$ (LFRGL) } \\
\hline \multicolumn{4}{|l|}{ T2-T1 } & \multicolumn{6}{|c|}{$\mathrm{p}=0,0019(\mathrm{LC}) ; \mathrm{p}=0,0443$ (LF); $\mathrm{p}=0,6452$ (LFRGL) } \\
\hline \multicolumn{4}{|l|}{ T2-T0 } & \multicolumn{6}{|c|}{$\mathrm{p}<0,0001$ (LC); $\mathrm{p}=0,0292$ (LF); $\mathrm{p}=0,4569$ (LFRGL) } \\
\hline \multicolumn{4}{|c|}{$\begin{array}{l}\text { Analyse der Wechselwirkung Gruppe } \\
\text { und Zeit }\end{array}$} & \multicolumn{6}{|c|}{$p=0,0453$} \\
\hline
\end{tabular}

Tabelle 8: Änderung des diastolischen Blutdrucks

\subsubsection{Vergleich der Blutdruckwerte}

Wie bereits in der Grafik zu sehen ist, besteht eine Tendenz zum Abfall der Blutdrücke in allen 3 Gruppen über die 6 Monate. Dieser Effekt ist mit -10 mm Hg systolisch in der Gruppe LC am größten, aber auch in Gruppe LF mit -4 mm Hg und LFRGL mit -2 mm Hg jeweils zumindest zwischen T0 und T2 mit p=0,0004 signifikant. Auch diastolisch kann in allen 3 Gruppen dieser Effekt über die Zeit beobachtet werden, verfehlt jedoch in der Gruppe LFRGL das Signifikanzniveau bei der Differenz T2-T0 mit -0,8 mm Hg. Währenddessen sind in den Gruppen LC und LF signifikante Blutdruckabnahmen zwischen T0 und T2 und zwischen T1 und T2 gemessen worden. Die Unterschiede der Blutdrucksenkung über die Interventionszeit zwischen den Gruppen lesen sich am p-Wert in der Analyse Wechselwirkung Gruppe und Zeit ab.

\subsubsection{Verwendung von Blutdruckmedikamenten}

In den Anamnesebögen zur Aufnahme in das Untersuchungsprogramm wurden alle Teilnehmerinnen gebeten, ihre regelmäßig eingenommenen Medikamente anzugeben, beziehungsweise keine, wenn sie keine einnahmen. 9 Frauen aus Gruppe LC, 10 aus Gruppe LF und 14 aus Gruppe LFRGL nahmen ein bis maximal 3 Antihypertensiva ein. Die erneute Befragung zur Medikamenteneinnahme nach 6 Monaten war leider nicht vollständig, da von 15 der 84 eingeschlossenen Probandinnen keine Rückmeldung diesbezüglich kam. Im Fall einer fehlenden Rückmeldung wurde keine Veränderung der Tabletteneinnahme angenommen. 
Die Auswertung ergab, dass eine Teilnehmerin aus Gruppe LC die Antihypertensiva ganz absetzen und 2 Teilnehmerinnen ihre Medikamente reduzieren konnten. Allerdings mussten auch 2 Frauen die Blutdruckmedikamente erhöhen. 2 der 10 Probandinnen mit antihypertensiver Therapie der Gruppe LF erreichten eine Reduktion ihrer Medikamente. In der Gruppe LFRGL konnte eine Patientin ihre Medikamente zur Senkung des Blutdrucks absetzen und eine weitere konnte sie reduzieren. Im Rahmen der Untersuchungen wurde jedoch bei einer Teilnehmerin dieser Gruppe ein Hypertonus neu diagnostiziert und entsprechend eine antihypertensive Therapie begonnen.

\subsection{Laboranalyse der Blutparameter}

\subsubsection{Homocystein, MTHFR und hoch sensitives C-reaktives Protein}

\subsubsection{Homocysteinwerte T0 und T1}

Die Messung der Homocysteinwerte wurde nur zu den Zeitpunkten T0 und T1 regulär für alle Teilnehmerinnen durchgeführt. Die Gründe dafür werden anschließend unter Punkt 3.4.1.2 dargelegt. Folgende Ergebnisse waren festzustellen:

\section{Homocystein in $\mu \mathrm{mol} / \mathrm{l}$}

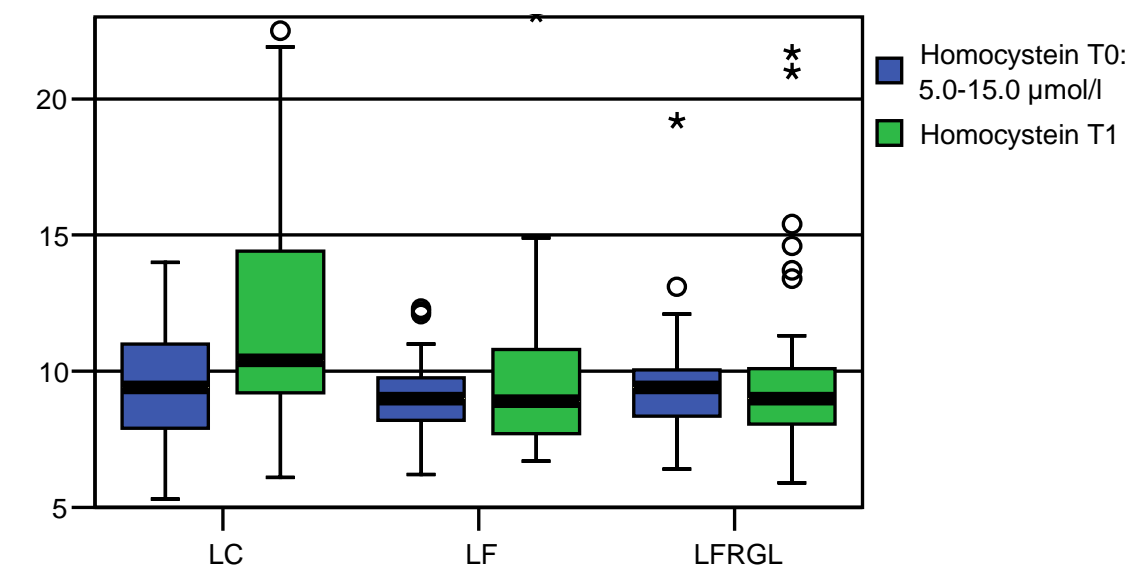

\begin{tabular}{|l|cc|cc|cc|}
\hline & \multicolumn{2}{|c|}{ Gruppe LC } & \multicolumn{2}{|c|}{ Gruppe LF } & \multicolumn{2}{c|}{ Gruppe LFRGL } \\
Zeitpunkt & T0 & T1 & T0 & T1 & T0 & T1 \\
\hline Mittel & 9,6 & 13,1 & 9,2 & 9,9 & 9,6 & 10,1 \\
Median & 9,4 & 10,4 & 9,0 & 8,9 & 9,4 & 9,0 \\
SD & 2,1 & 6,0 & 1,5 & 3,3 & 2,3 & 3,8 \\
Min. & 5,3 & 6,1 & 6,2 & 6,7 & 6,4 & 5,9 \\
Max. & 14,0 & 30,8 & 12,3 & 23,1 & 19,2 & 21,7 \\
\hline
\end{tabular}




\begin{tabular}{|l|c|c|c|}
\hline & Gruppe LC & Gruppe LF & Gruppe LFRGL \\
Zeitpunkt & T1-T0 & T2-T1 & T2-T0 \\
\hline Mittel & 3,6 & 0,7 & 0,5 \\
Median & 0,6 & 0,0 & $-0,2$ \\
SD & 6,8 & 3,4 & 2,6 \\
Min. & $-2,4$ & $-3,0$ \\
Max. & 21,5 & & 11,9 \\
\hline Analyse der Gruppenunterschiede & $\mathrm{p}=0,0144$ (LC); $\mathrm{p}=0,6896$ (LF); $\mathrm{p}=0,5255$ (LFRGL) \\
Analyse der Zeitdifferenz & $\mathrm{p}=0,0410$ \\
Analyse der Wechselwirkung Gruppe und & & \\
Zeit &
\end{tabular}

Tabelle 9: Homocysteinänderung T0 bis T1

\subsubsection{Die Zwischenblutabnahme des Homocysteins zum Zeitpunkt T1a}

Die relevanten Homocysteinerhöhungen bei einigen Teilnehmerinnen veranlassten uns zu einer Zwischenblutabnahme nach 3 Monaten Diät bei allen Frauen mit Homocysteinanstieg > $5 \mu \mathrm{mol} / 1$ sowie bei allen Probandinnen der Gruppe LC, aufgrund des allgemeinen Anstiegs dort. Das Ergebnis zeigt, dass bei allen Teilnehmerinnen bis auf eine aus der Gruppe LC die Werte - fast - wieder auf das Ausgangsniveau gesunken waren. Die Werte der 30 Teilnehmerinnen und die Differenzen der gesamten Homocysteinwerte sind folgende:

\section{Homocysteinwerte von T0 bis T1a}

\begin{tabular}{|l|ccc|ccc|}
\hline & \multicolumn{3}{|c}{ Homocystein } & \multicolumn{3}{|c|}{ Differenzen } \\
Zeitpunkt & T0 & T1 & T1a & T1-T0 & T1a-T1 & T1a-T0 \\
\hline Mittel & 9,9 & 14,1 & 10,3 & 4,2 & $-3,8$ & 0,4 \\
Median & 9,6 & 11,4 & 9,2 & 0,8 & $-0,6$ & $-0,1$ \\
SD & 2,7 & 6,2 & 3,6 & 6,8 & 6,6 & 3,4 \\
Min. & 5,3 & 6,1 & 5,7 & $-2,4$ & $-22,0$ & $-3,3$ \\
Max. & 19,2 & 30,8 & 22,4 & 21,5 & 3,4 & 17,1 \\
\hline
\end{tabular}

Tabelle 10:Homocysteinänderung T0 bis T1a

\subsubsection{MTHFR-Genotyp}

Der deutliche Homocysteinanstieg zum Zeitpunkt T1 veranlasste uns auch zur Bestimmung des Genotyps bezüglich des Enzyms Methylen-Tetrahydrofolat-Reduktase (MTHFR) bei den Probandinnen der Kontrollblutabnahme T1a. Damit sollten mögliche Zusammenhänge zwischen Homocysteinanstieg und dem Vorhandensein eines bestimmten MTHFR-

Genotyps geklärt werden. Wir konnten von 29 der 30 Probandinnen dieser Kontrollgruppe den Genotyp ermitteln. Es gibt den so genannten Wildtyp CC mit zwei normalen Allelen, den 
Mischtyp CT mit einem normalen und einem veränderten Allel und den TT-Genotyp mit zwei veränderten Allelen. Folgendes Ergebnis zeigte sich:

Ergebnis der MTHFR-Genotyp-Bestimmung

\begin{tabular}{|l|c|c|}
\hline Genotyp & Häufigkeit & Prozent \\
\hline CC & 19 & 63,3 \\
CT & 8 & 26,7 \\
TT & 2 & 6,7 \\
Fehlend & 1 & 3,3 \\
Gesamt & 30 & 100 \\
\hline
\end{tabular}

Tabelle 11:Verteilung der MTHFR-Genotypen

\subsubsection{Hoch sensitives CRP (hsCRP)}

\section{HsCRP in mg/l}

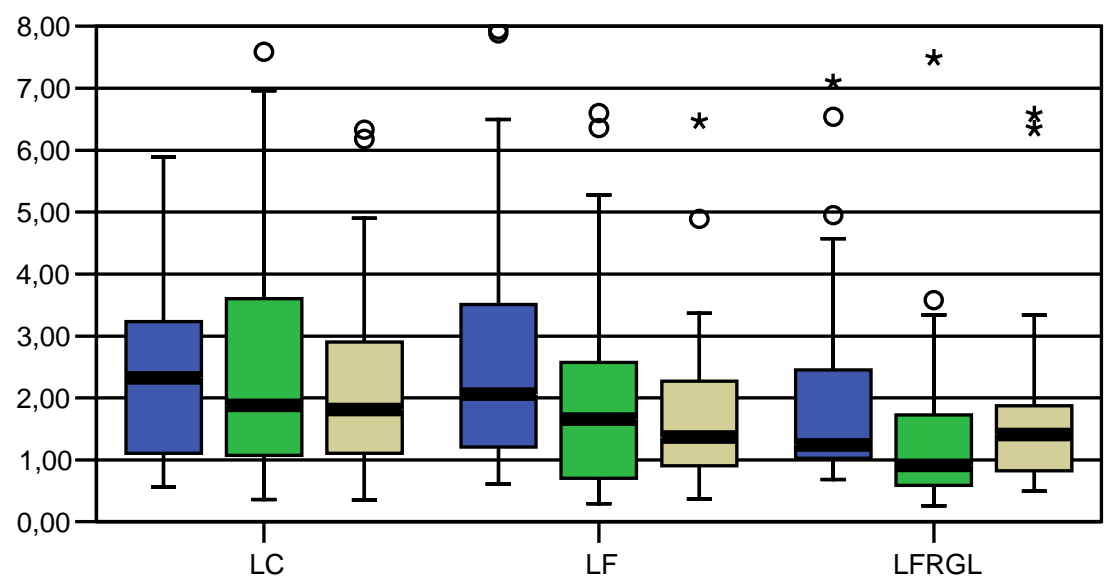

hsCRP T0: $<2.0$

$\square$ hsCRP T1

$\square$ hsCRP T2

\begin{tabular}{|l|ccc|ccc|ccc|}
\hline & \multicolumn{3}{|l}{ Gruppe LC } & \multicolumn{4}{|c|}{ Gruppe LF } & \multicolumn{3}{|c|}{ Gruppe LFRGL } \\
Zeitpunkt & T0 & T1 & T2 & T0 & T1 & T2 & T0 & T1 & T2 \\
\hline Mittel & 2,34 & 2,68 & 2,30 & 2,70 & 2,08 & 1,83 & 2,12 & 1,45 & 1,73 \\
Median & 2,33 & 1,88 & 1,82 & 2,06 & 1,66 & 1,37 & 1,24 & 0,91 & 1,41 \\
SD & 1,39 & 2,01 & 1,64 & 2,09 & 1,80 & 1,39 & 1,69 & 1,42 & 1,45 \\
Min. & 0,56 & 0,36 & 0,35 & 0,61 & 0,29 & 0,37 & 0,68 & 0,26 & 0,49 \\
Max. & 5,89 & 7,59 & 6,33 & 7,95 & 6,60 & 6,47 & 7,10 & 7,49 & 6,58 \\
\hline
\end{tabular}

\begin{tabular}{|l|rrr|rrr|rrr|}
\hline & \multicolumn{4}{|l}{ Gruppe LC } & \multicolumn{4}{l|}{ Gruppe LF } & \multicolumn{3}{|c|}{ Gruppe LFRGL } \\
Differenz & T1-T0 & T2-T1 & T2-T0 & T1-T0 & T2-T1 & T2-T0 & T1-T0 & T2-T1 & T2-T0 \\
\hline Mittel & 0,34 & $-0,38$ & $-0,04$ & $-0,62$ & $-0,25$ & $-0,87$ & $-0,67$ & 0,28 & $-0,39$ \\
Median & 0,11 & $-0,23$ & $-0,27$ & $-0,21$ & $-0,04$ & $-0,30$ & $-0,53$ & 0,40 & $-0,22$ \\
SD & 2,18 & 2,10 & 1,99 & 1,86 & 1,12 & 1,47 & 0,73 & 0,88 & 0,92 \\
Min. & $-3,23$ & $-5,73$ & $-3,30$ & $-7,35$ & $-3,97$ & $-6,05$ & $-2,96$ & $-2,71$ & $-4,02$ \\
Max. & 5,54 & 5,27 & 5,50 & 2,07 & 1,30 & 0,81 & 0,39 & 3,00 & 1,56 \\
\hline
\end{tabular}

Tabelle 12:HsCRP-Veränderungen 
Die Messergebnisse für das hsCRP lagen außerhalb einer Normalverteilungskurve. Aus diesem Grund wurde für die statistischen Tests und Korrelationen zu den anderen Parametern der natürliche Logarithmus jedes einzelnen Messwertes gebildet. Dies ist ein häufig gebrauchtes Verfahren bei der Verwendung von hsCRP-Werten in Studien aufgrund der bekannt breiten Streuung der Werte. Es war trotz des Ausschlusses aller Probandinnen mit hsCRP-Werten $>8 \mathrm{mg} / \mathrm{l}$ notwendig. Anschließend zeigten sich die folgenden Ergebnisse normalverteilt:

\section{Natürlicher Logarithmus des hsCRP in mg/l}

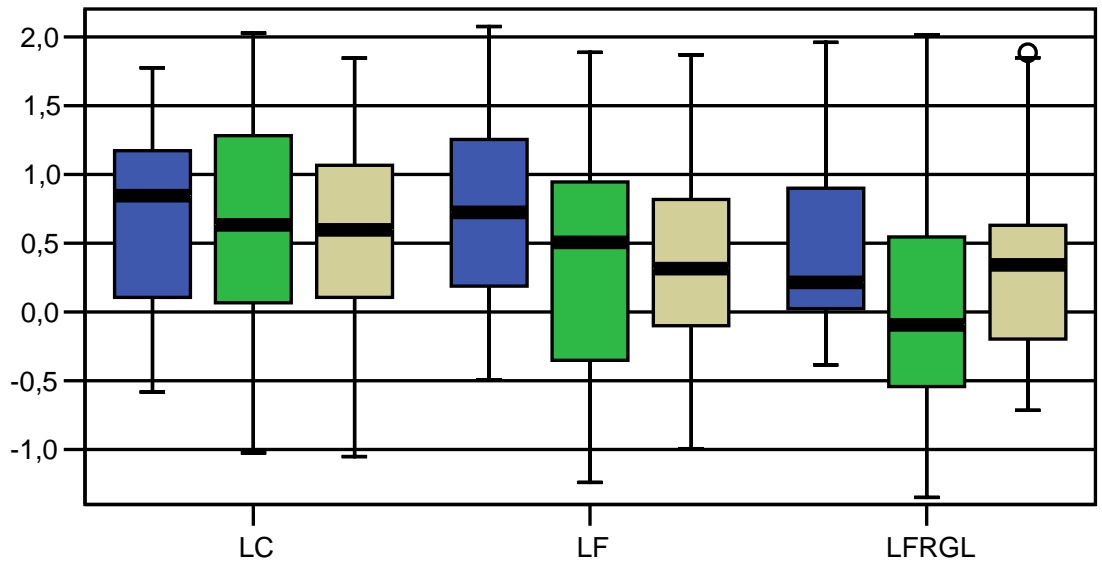

\begin{tabular}{|l|ccc|ccc|ccc|}
\hline & \multicolumn{2}{|l}{ Gruppe LC } & \multicolumn{2}{|c|}{ Gruppe LF } & & \multicolumn{2}{c|}{ Gruppe FRGL } \\
Zeitpunkt & T0 & T1 & T2 & T0 & T1 & T2 & T0 & T1 & T2 \\
\hline Mittel & 0,66 & 0,72 & 0,58 & 0,71 & 0,38 & 0,36 & 0,51 & 0,06 & 0,31 \\
Median & 0,84 & 0,63 & 0,60 & 0,72 & 0,51 & 0,31 & 0,22 & $-0,09$ & 0,34 \\
SD & 0,67 & 0,77 & 0,77 & 0,77 & 0,86 & 0,71 & 0,67 & 0,76 & 0,66 \\
Min. & $-0,58$ & $-1,02$ & $-1,05$ & $-0,49$ & $-1,24$ & $-0,99$ & $-0,39$ & $-1,35$ & $-0,71$ \\
Max. & 1,77 & 2,03 & 1,85 & 2,07 & 1,89 & 1,87 & 1,96 & 2,01 & 1,88 \\
\hline
\end{tabular}

\begin{tabular}{|c|c|c|c|c|c|c|c|c|c|}
\hline \multirow[b]{2}{*}{ Differenz } & \multicolumn{3}{|c|}{ Gruppe LC } & \multicolumn{3}{|c|}{ Gruppe LF } & \multicolumn{3}{|c|}{ Gruppe FRGL } \\
\hline & T1-T0 & T2-T1 & T2-Т0 & T1-T0 & T2-T1 & T2-Т0 & T1-T0 & T2-T1 & T2-T0 \\
\hline Mittel & 0,06 & $-0,14$ & $-0,08$ & $-0,33$ & $-0,02$ & $-0,35$ & $-0,45$ & 0,25 & $-0,20$ \\
\hline Median & 0,09 & $-0,15$ & $-0,25$ & $-0,09$ & $-0,02$ & $-0,22$ & $-0,49$ & 0,38 & $-0,18$ \\
\hline SD & 0,81 & 0,77 & 0,79 & 0,79 & 0,53 & 0,55 & 0,33 & 0,53 & 0,49 \\
\hline Min. & $-1,35$ & $-2,71$ & $-1,26$ & $-2,68$ & $-1,39$ & $-1,74$ & $-1,06$ & $-1,78$ & $-2,12$ \\
\hline Max. & 2,33 & 1,92 & 2,21 & 0,60 & 1,23 & 0,43 & 0,25 & 0,93 & 0,81 \\
\hline \multicolumn{10}{|c|}{ Analyse der Gruppenunterschiede } \\
\hline \multicolumn{4}{|c|}{ Analyse der Zeitdifferenz } & \multicolumn{6}{|c|}{$p<0,6663(L C) ; p=0,0112(L F) ; p<0,0001$ (LFRGL) } \\
\hline \multicolumn{4}{|l|}{ T1-T0 } & \multicolumn{6}{|c|}{$p=0,7044(L C) ; p=0,0412(L F) ; p<0,0001$ (LFRGL) } \\
\hline \multicolumn{4}{|l|}{ T2-T1 } & \multicolumn{6}{|c|}{$\mathrm{p}=0,3657(\mathrm{LC}) ; \mathrm{p}=0,8222(\mathrm{LF}) ; \mathrm{p}=0,0135$ (LFRGL) } \\
\hline \multicolumn{4}{|l|}{ T2-T0 } & \multicolumn{6}{|c|}{$p=0,6182(L C) ; p=0,0027$ (LF); $p=0,0292(L F R G L)$} \\
\hline \multicolumn{4}{|c|}{$\begin{array}{l}\text { Analyse der Wechselwirkung Gruppe } \\
\text { und Zeit }\end{array}$} & \multicolumn{6}{|c|}{$p=0,0299$} \\
\hline
\end{tabular}

Tabelle 13:Differenz der natürlich logarithmierten hsCRP-Werte 


\subsubsection{Vergleich der Werte für Homocystein, MTHFR und hsCRP}

Der Anstieg des Homocysteins in der Gruppe LC wird in der grafischen Darstellung gut sichtbar. Auch wenn die Streuung der Einzelwerte in der zweiten Messung hier deutlich gröBer ist, bleibt der mittlere Anstieg mit p=0,0144 signifikant. Dahingegen sind die Anstiege der Homocysteinwerte in den Gruppen LF und LFRGL nicht signifikant. Betrachtet man die Einzelwerte genauer, lässt sich der große Anstieg des Mittelwerts in Gruppe LC nicht nur auf viele kleine Erhöhungen zurückführen. Bei 6 der Teilnehmerinnen aus dieser Gruppe stieg das Homocystein auf fast den doppelten Ausgangswert an. Dies war bei nur einer Teilnehmerin in Gruppe LF und bei 2 Teilnehmerinnen in Gruppe LFRGL der Fall.

Betrachtet man die Homocysteinwerte der 30 Teilnehmerinnen der Zwischenblutabnahme T1a sieht man, dass der Anstieg von zunächst 4,2 $\mu$ mol/l bis T1 interessanterweise im Zeitraum von T1 zu T1a mit -3,8 fast komplett reversibel war. Nach dieser Kontrolle der Homocysteinwerte empfahlen wir allen Teilnehmerinnen des Programms mit Werten $>12 \mu \mathrm{mol} / \mathrm{l}$ die Einnahme von Vitaminsupplementen zur Substitution von Folsäure, Vitamin B6 und B12. Diese Maßnahme verfügten wir zum Schutz unserer Probandinnen nach den Empfehlungen der Literatur. Da durch die Vitaminsupplemente mögliche weitere Effekte der Diäten auf die Homocysteinentwicklung überdeckt werden könnten, verzichteten wir auf die geplante Kontrolle der Werte zum Zeitpunkt T2.

Die hsCRP-Werte sinken in der Gruppe LF kontinuierlich und statistisch signifikant über die 3 Zeitpunkte ab, während in der Gruppe LC die Absenkung mit -0,08 $\mu \mathrm{mol} / \mathrm{l}$ die Signifikanz verfehlt. Hingegen sieht man in der Gruppe LFRGL zunächst einen deutlichen Abfall und anschließend einen leichten Wideranstieg des hsCRP. Letztlich bleibt die Abnahme des Wertes zwischen T0 und T2 in dieser Gruppe dennoch signifikant. 


\subsubsection{Glucose und Blutfette}

\subsubsection{Laborergebnisse}

\section{Glucose in mg/dl}

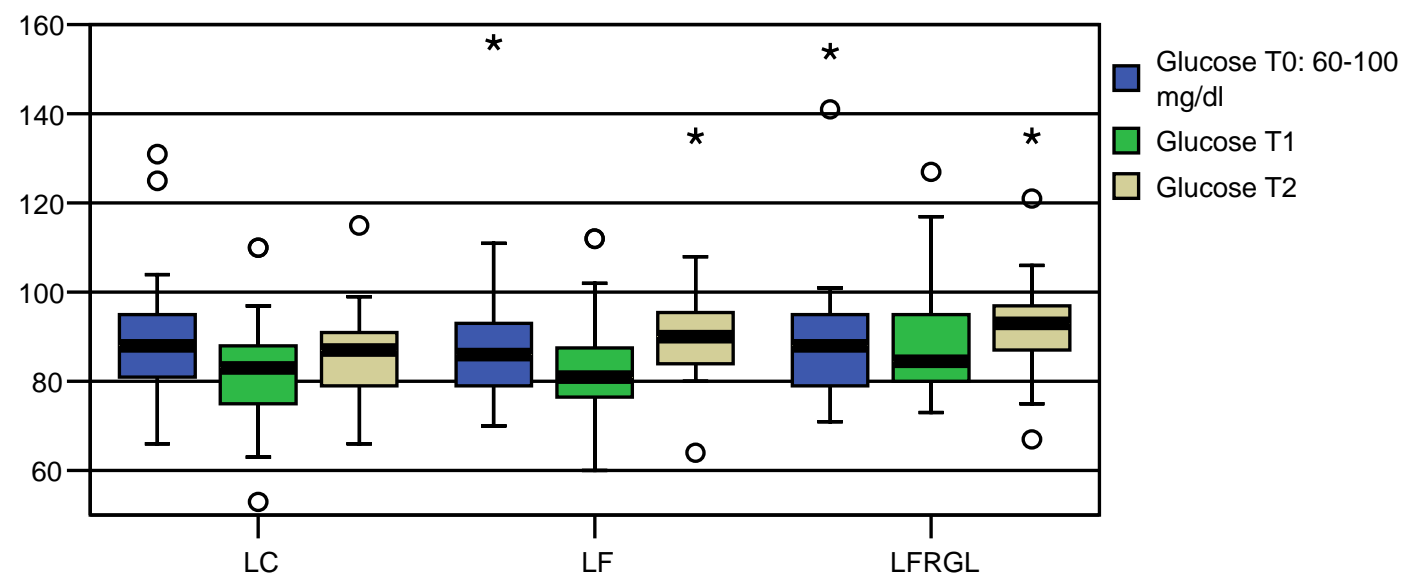

\begin{tabular}{|l|ccc|ccc|ccc|}
\hline & \multicolumn{3}{|l}{ Gruppe LC } & \multicolumn{3}{|c|}{ Gruppe LF } & \multicolumn{3}{|c|}{ Gruppe LFRGL } \\
Zeitpunkt & T0 & T1 & T2 & T0 & T1 & T2 & T0 & T1 & T2 \\
\hline Mittel & 89,6 & 82,1 & 86,3 & 89,0 & 83,6 & 91,5 & 89,8 & 88,6 & 93,2 \\
Median & 88,0 & 83,0 & 87,0 & 86,0 & 81,0 & 90,0 & 88,0 & 84,5 & 93,0 \\
SD & 14,9 & 12,9 & 10,6 & 16,9 & 12,4 & 12,7 & 17,9 & 12,9 & 12,5 \\
Min. & 66,0 & 53,0 & 66,0 & 70,0 & 60,0 & 64,0 & 71,0 & 73,0 & 67,0 \\
Max. & 131,0 & 110,0 & 115,0 & 156,0 & 112,0 & 135,0 & 154,0 & 127,0 & 135,0 \\
\hline
\end{tabular}

\begin{tabular}{|c|c|c|c|c|c|c|c|c|c|}
\hline \multirow[b]{2}{*}{ Differenz } & \multicolumn{3}{|c|}{ Gruppe LC } & \multicolumn{3}{|c|}{ Gruppe LF } & \multicolumn{3}{|c|}{ Gruppe FRGL } \\
\hline & T1-T0 & $\mathrm{T} 2-\mathrm{T} 1$ & T2-T0 & T1-T0 & $\mathrm{T} 2-\mathrm{T} 1$ & T2-T0 & T1-T0 & $\mathrm{T} 2-\mathrm{T} 1$ & T2-T0 \\
\hline Mittel & $-7,4$ & 4,2 & $-3,3$ & $-5,5$ & 8,0 & 2,5 & $-1,2$ & 4,7 & 3,4 \\
\hline Median & $-7,0$ & 3,0 & $-3,0$ & $-4,0$ & 8,0 & 4,0 & 0,0 & 5,5 & 5,5 \\
\hline SD & 7,1 & 7,4 & 9,4 & 10,3 & 9,1 & 9,4 & 10,9 & 8,0 & 10,7 \\
\hline Min. & $-21,0$ & $-15,0$ & $-30,0$ & $-44,0$ & $-8,0$ & $-21,0$ & $-37,0$ & $-10,0$ & $-20,0$ \\
\hline Max. & 3,0 & 20,0 & 13,0 & 11,0 & 28,0 & 21,0 & 23,0 & 18,0 & 20,0 \\
\hline \multicolumn{10}{|c|}{ Analyse der Gruppenunterschiede } \\
\hline \multicolumn{4}{|c|}{ Analyse der Zeitdifferenz } & \multicolumn{6}{|c|}{$\mathrm{p}<0,0001$ (LC); $\mathrm{p}<0,0001$ (LF); $\mathrm{p}=0,0338$ (LFRGL) } \\
\hline \multicolumn{4}{|l|}{ T1-T0 } & \multicolumn{6}{|c|}{$\mathrm{p}<0,0001$ (LC); $\mathrm{p}=0,0106$ (LF); $\mathrm{p}=0,5392$ (LFRGL) } \\
\hline \multicolumn{4}{|l|}{$\mathrm{T} 2-\mathrm{T} 1$} & \multicolumn{6}{|c|}{$\mathrm{p}=0,0094(\mathrm{LC}) ; \mathrm{p}=0,0001$ (LF); $\mathrm{p}=0,0034$ (LFRGL) } \\
\hline \multicolumn{4}{|l|}{ T2-T0 } & \multicolumn{6}{|c|}{$\mathrm{p}=0,0946$ (LC); $\mathrm{p}=0,1820$ (LF); $\mathrm{p}=0,0891$ (LFRGL) } \\
\hline \multicolumn{4}{|c|}{$\begin{array}{l}\text { Analyse der Wechselwirkung Gruppe } \\
\text { und Zeit }\end{array}$} & \multicolumn{6}{|c|}{$p=0,0215$} \\
\hline
\end{tabular}

Tabelle 14: Änderung der Glucose 
Triglyceride in mg/dl

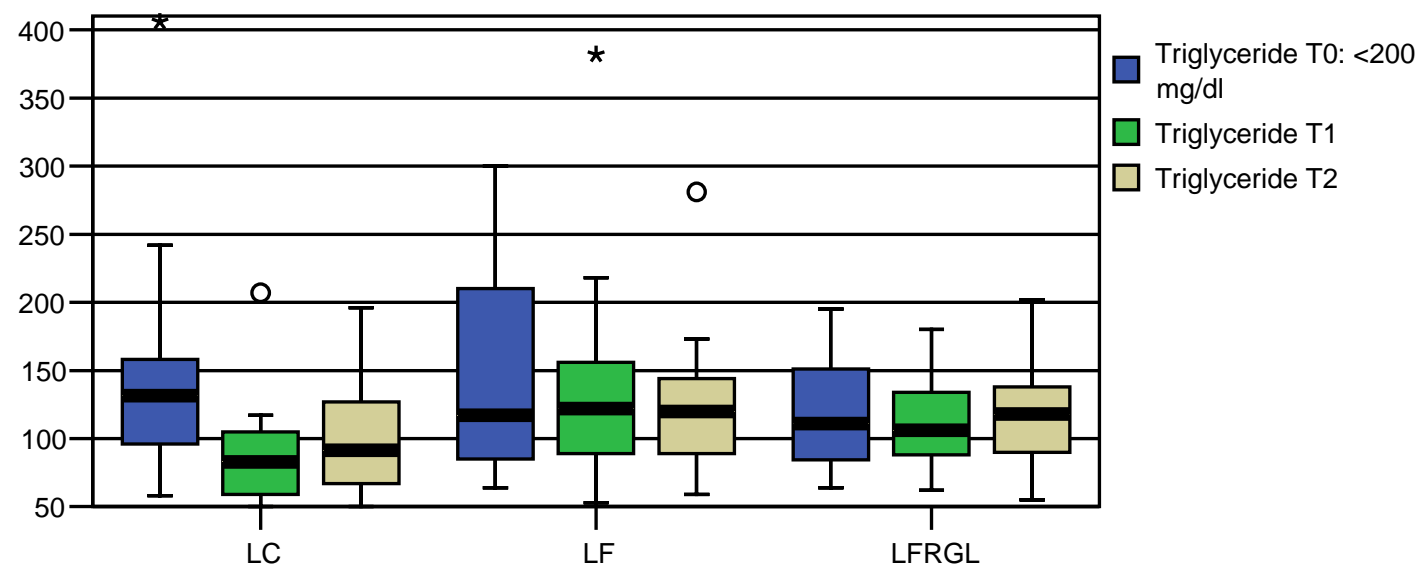

\begin{tabular}{|l|ccc|ccc|ccc|}
\hline & \multicolumn{3}{|l}{ Gruppe LC } & \multicolumn{4}{|l|}{ Gruppe LF } & \multicolumn{3}{|c|}{ Gruppe LFRGL } \\
Zeitpunkt & T0 & T1 & T2 & T0 & T1 & T2 & T0 & T1 & T2 \\
\hline Mittel & 141,5 & 85,9 & 98,8 & 141,8 & 131,2 & 121,6 & 117,5 & 111,5 & 117,9 \\
Median & 131,5 & 83,0 & 91,5 & 116,0 & 122,0 & 113,0 & 111,0 & 106,0 & 118,0 \\
SD & 73,4 & 33,1 & 42,3 & 71,3 & 65,3 & 44,5 & 38,4 & 31,2 & 39,6 \\
Min. & 58,0 & 50,0 & 36,0 & 64,0 & 53,0 & 59,0 & 64,0 & 62,0 & 55,0 \\
Max. & 406,0 & 207,0 & 196,0 & 300,0 & 382,0 & 281,0 & 195,0 & 180,0 & 202,0 \\
\hline
\end{tabular}

\begin{tabular}{|c|c|c|c|c|c|c|c|c|c|}
\hline \multirow[b]{2}{*}{ Differenz } & \multicolumn{3}{|c|}{ Gruppe LC } & \multicolumn{3}{|c|}{ Gruppe LF } & \multicolumn{3}{|c|}{ Gruppe LFRGL } \\
\hline & T1-T0 & $\mathrm{T} 2-\mathrm{T} 1$ & T2-T0 & T1-T0 & $\mathrm{T} 2-\mathrm{T} 1$ & T2-Т0 & T1-T0 & $\mathrm{T} 2-\mathrm{T} 1$ & T2-T0 \\
\hline Mittel & $-55,6$ & 12,9 & $-42,7$ & $-12,0$ & $-8,4$ & $-20,2$ & $-6,0$ & 6,5 & 0,4 \\
\hline Median & $-42,0$ & 3,0 & $-37,5$ & $-5,0$ & $-14,0$ & $-13,0$ & $-5,0$ & 0,0 & 1,0 \\
\hline SD & 62,8 & 39,9 & 63,6 & 57,0 & 42,4 & 51,6 & 33,0 & 28,9 & 34,9 \\
\hline Min. & $-299,0$ & $-81,0$ & $-266,0$ & $-153,0$ & $-101,0$ & $-127,0$ & $-72,0$ & $-35,0$ & $-63,0$ \\
\hline Max. & 51,0 & 91,0 & 75,0 & 100,0 & 65,0 & 58,0 & 59,0 & 83,0 & 85,0 \\
\hline \multicolumn{10}{|c|}{ Analyse der Gruppenunterschiede } \\
\hline \multicolumn{4}{|c|}{ Analyse der Zeitdifferenz } & \multicolumn{6}{|c|}{$p<0,0001(L C) ; p=0,7819$ (LF); $p=0,6768$ (LFRGL) } \\
\hline \multicolumn{4}{|l|}{ T1-T0 } & \multicolumn{6}{|c|}{$\mathrm{p}<0,0001$ (LC); $\mathrm{p}=0,6904$ (LF); $\mathrm{p}=0,5127$ (LFRGL) } \\
\hline \multicolumn{4}{|l|}{ T2-T1 } & \multicolumn{6}{|c|}{$\mathrm{p}=0,0594(\mathrm{LC}) ; \mathrm{p}=0,7869$ (LF); $\mathrm{p}=0,3855$ (LFRGL) } \\
\hline \multicolumn{4}{|l|}{ T2-T0 } & \multicolumn{6}{|c|}{$\mathrm{p}=0,0022(\mathrm{LC}) ; \mathrm{p}=0,4670$ (LF); $\mathrm{p}=0,9617$ (LFRGL) } \\
\hline \multicolumn{4}{|c|}{$\begin{array}{l}\text { Analyse der Wechselwirkung Gruppe } \\
\text { und Zeit }\end{array}$} & \multicolumn{6}{|c|}{$P<0,0001$} \\
\hline
\end{tabular}

Tabelle 15: Änderung der Triglyceride 


\section{Gesamtcholesterin in $\mathbf{m g} / \mathbf{d l}$}

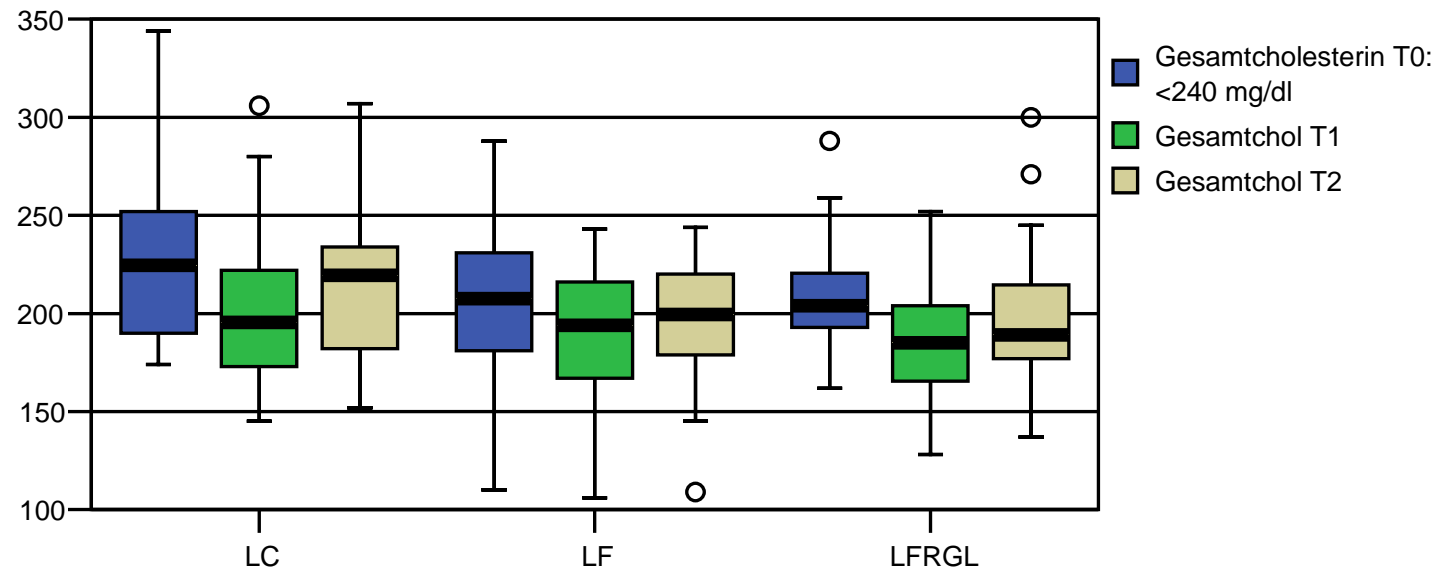

\begin{tabular}{|l|ccc|ccc|ccc|}
\hline & \multicolumn{2}{|l}{ Gruppe LC } & \multicolumn{3}{|c|}{ Gruppe LF } & & \multicolumn{2}{c|}{ Gruppe LFRGL } \\
Zeitpunkt & T0 & T1 & T2 & T0 & T1 & T2 & T0 & T1 & T2 \\
\hline Mittel & 224,8 & 204,6 & 215,3 & 208,1 & 191,6 & 196,4 & 207,0 & 184,1 & 196,6 \\
Median & 224,5 & 195,5 & 219,5 & 210,0 & 194,0 & 200,0 & 204,0 & 185,0 & 189,0 \\
SD & 40,3 & 40,7 & 42,4 & 38,8 & 35,1 & 31,5 & 29,6 & 28,6 & 34,1 \\
Min. & 174,0 & 145,0 & 152,0 & 110,0 & 106,0 & 109,0 & 162,0 & 128,0 & 137,0 \\
Max. & 344,0 & 306,0 & 307,0 & 288,0 & 243,0 & 244,0 & 288,0 & 252,0 & 300,0 \\
\hline
\end{tabular}

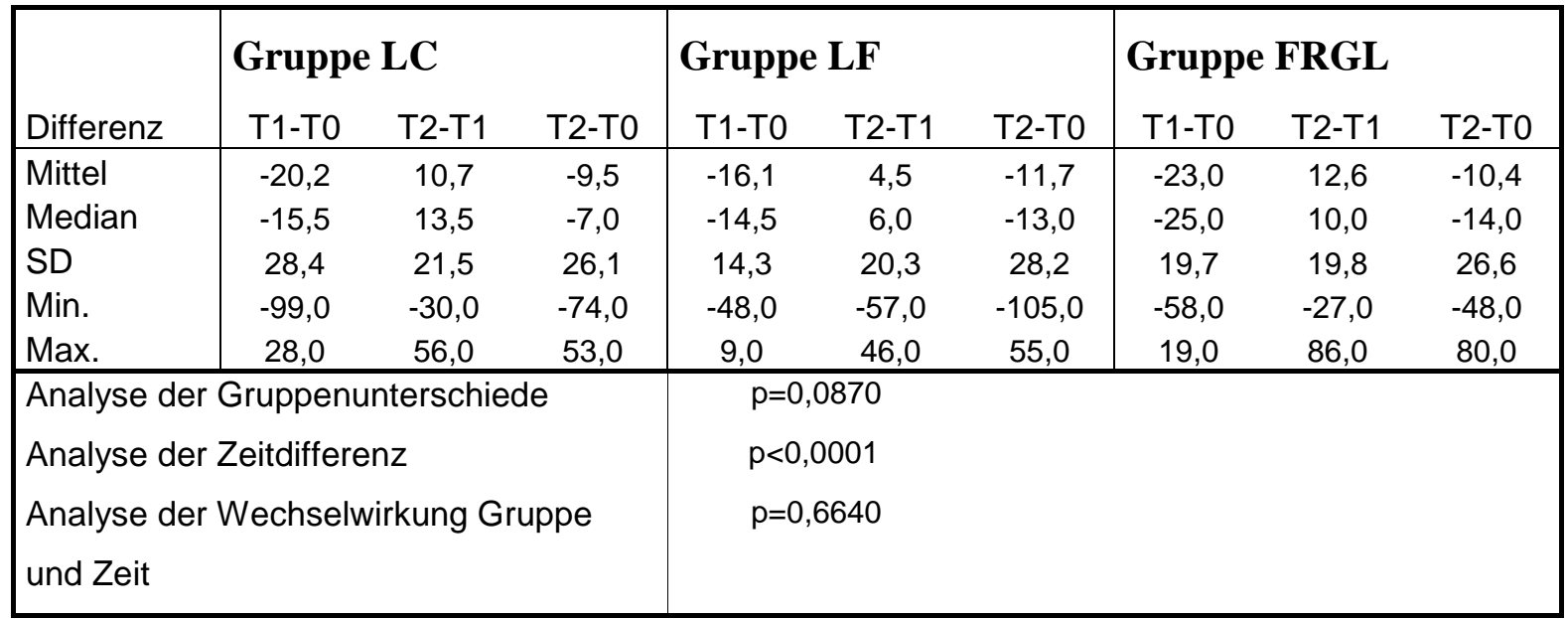

Tabelle 16: Änderung des Gesamtcholesterins 


\section{LDL-Cholesterin in $\mathbf{m g} / \mathbf{d l}$}

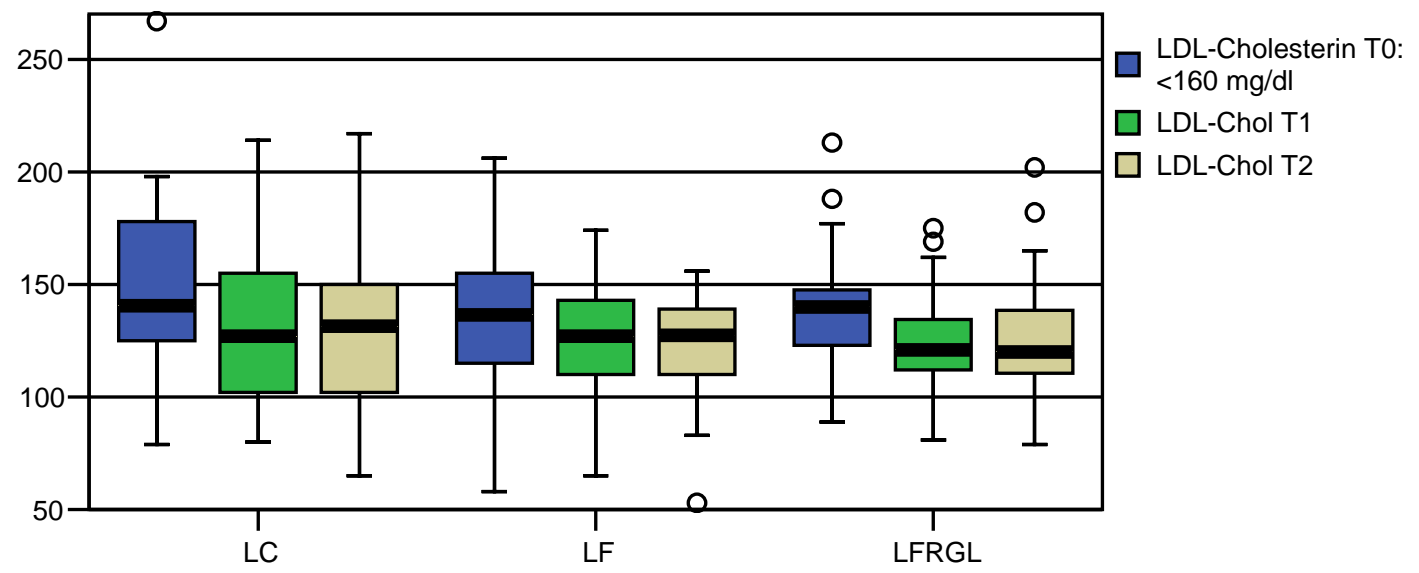

\begin{tabular}{|l|ccc|ccc|ccc|}
\hline & \multicolumn{2}{|c}{ Gruppe LC } & \multicolumn{3}{|c|}{ Gruppe LF } & & \multicolumn{3}{c|}{ Gruppe LFRGL } \\
Zeitpunkt & T0 & T1 & T2 & T0 & T1 & T2 & T0 & T1 & T2 \\
\hline Mittel & 147,0 & 130,7 & 131,2 & 136,8 & 128,2 & 122,4 & 138,9 & 124,0 & 125,4 \\
Median & 140,5 & 127,0 & 131,5 & 132,0 & 127,0 & 127,0 & 140,0 & 121,0 & 120,0 \\
SD & 39,5 & 35,9 & 35,2 & 33,4 & 27,3 & 24,4 & 25,7 & 23,0 & 26,6 \\
Min. & 79,0 & 80,0 & 65,0 & 58,0 & 65,0 & 53,0 & 89,0 & 81,0 & 79,0 \\
Max. & 267,0 & 214,0 & 217,0 & 206,0 & 174,0 & 156,0 & 213,0 & 175,0 & 202,0 \\
\hline
\end{tabular}

\begin{tabular}{|c|c|c|c|c|c|c|c|c|c|}
\hline \multirow[b]{2}{*}{ Differenz } & \multicolumn{3}{|c|}{ Gruppe LC } & \multicolumn{3}{|c|}{ Gruppe LF } & \multicolumn{3}{|c|}{ Gruppe LFRGL } \\
\hline & T1-T0 & T2-T1 & T2-T0 & T1-T0 & T2-T1 & T2-T0 & T1-T0 & T2-T1 & T2-T0 \\
\hline Mittel & $-16,3$ & 0,5 & $-15,8$ & $-9,2$ & $-5,0$ & $-14,3$ & $-15,0$ & 1,4 & $-13,5$ \\
\hline Median & $-14,0$ & 3,0 & $-13,0$ & $-11,0$ & $-4,0$ & $-13,0$ & $-11,0$ & 0,0 & $-18,0$ \\
\hline SD & 23,5 & 16,8 & 19,3 & 13,9 & 17,3 & 23,7 & 16,7 & 14,9 & 19,3 \\
\hline Min. & $-78,0$ & $-27,0$ & $-66,0$ & $-33,0$ & $-58,0$ & $-91,0$ & $-44,0$ & $-23,0$ & $-48,0$ \\
\hline Max. & 18,0 & 37,0 & 23,0 & 22,0 & 25,0 & 33,0 & 16,0 & 40,0 & 38,0 \\
\hline \multicolumn{4}{|c|}{ Analyse der Gruppenunterschiede } & \multicolumn{2}{|c|}{$p=0,5613$} & & & & \\
\hline \multicolumn{4}{|c|}{ Analyse der Zeitdifferenz } & \multicolumn{2}{|c|}{$p<0,0001$} & & & & \\
\hline \multirow{2}{*}{\multicolumn{4}{|c|}{$\begin{array}{l}\text { Analyse der Wechselwirkung Gruppe } \\
\text { und Zeit }\end{array}$}} & \multirow{2}{*}{\multicolumn{2}{|c|}{$p=0,5354$}} & & & & \\
\hline & & & & & & & & & \\
\hline
\end{tabular}

Tabelle 17: Änderung des LDL-Cholesterins 
HDL-Cholesterin in $\mathbf{m g} / \mathrm{dl}$

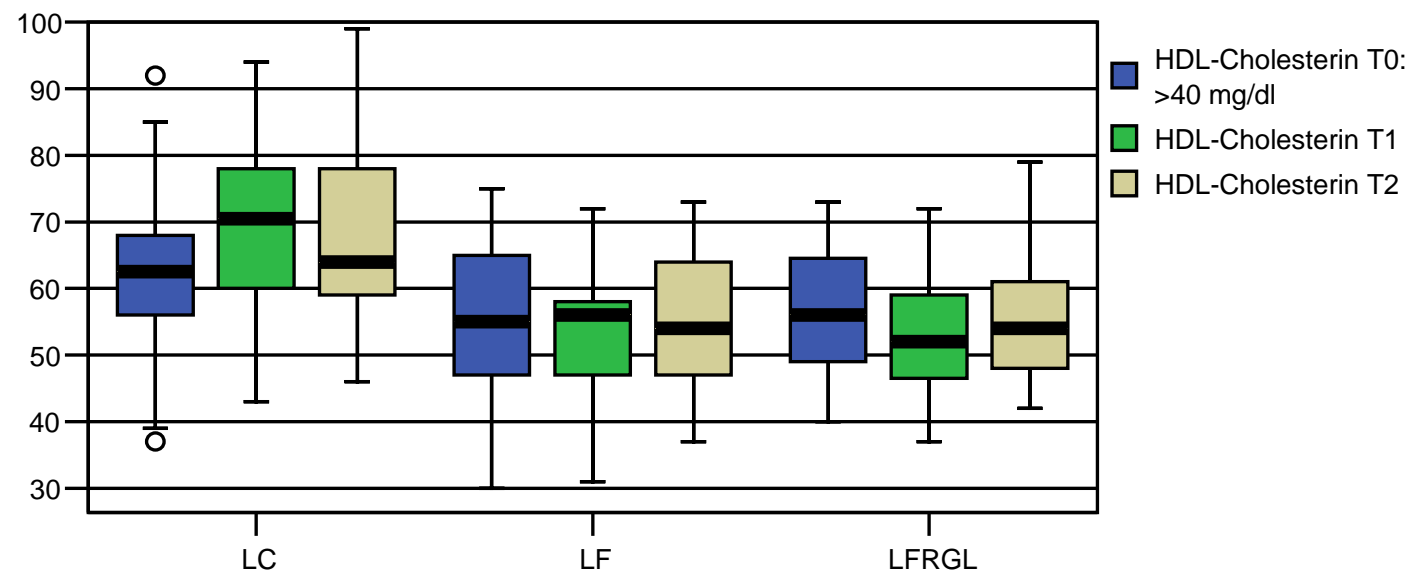

\begin{tabular}{|l|ccc|ccc|ccc|}
\hline & \multicolumn{3}{|c|}{ Gruppe LC } & \multicolumn{3}{|c|}{ Gruppe LF } & \multicolumn{3}{|c|}{ Gruppe LFRGL } \\
Zeitpunkt & T0 & T1 & T2 & T0 & T1 & T2 & T0 & T1 & T2 \\
\hline Mittel & 63,3 & 70,0 & 67,0 & 57,4 & 53,4 & 56,6 & 56,2 & 52,8 & 55,5 \\
Median & 62,5 & 70,5 & 64,0 & 55,0 & 56,0 & 56,0 & 56,0 & 52,0 & 54,0 \\
SD & 13,1 & 13,8 & 13,5 & 12,6 & 10,8 & 12,6 & 9,4 & 9,5 & 9,3 \\
Min. & 37,0 & 43,0 & 46,0 & 30,0 & 31,0 & 37,0 & 40,0 & 37,0 & 42,0 \\
Max. & 92,0 & 94,0 & 99,0 & 89,0 & 72,0 & 92,0 & 73,0 & 72,0 & 79,0 \\
\hline
\end{tabular}

\begin{tabular}{|c|c|c|c|c|c|c|c|c|c|}
\hline \multirow[b]{2}{*}{ Differenz } & \multicolumn{3}{|c|}{ Gruppe LC } & \multicolumn{3}{|c|}{ Gruppe LF } & \multicolumn{3}{|c|}{ Gruppe LFRGL } \\
\hline & T1-T0 & $\mathrm{T} 2-\mathrm{T} 1$ & T2-T0 & T1-T0 & $\mathrm{T} 2-\mathrm{T} 1$ & T2-T0 & T1-T0 & $\mathrm{T} 2-\mathrm{T} 1$ & T2-T0 \\
\hline Mittel & 6,7 & $-3,0$ & 3,7 & $-2,7$ & 1,8 & $-0,8$ & $-3,3$ & 2,7 & $-0,6$ \\
\hline Median & 7,0 & $-1,5$ & 1,0 & $-4,5$ & 1,0 & 0,0 & $-3,0$ & 2,0 & $-2,0$ \\
\hline SD & 8,4 & 9,6 & 11,7 & 6,7 & 6,0 & 6,2 & 5,6 & 5,6 & 8,0 \\
\hline Min. & $-7,0$ & $-30,0$ & $-20,0$ & $-15,0$ & $-10,0$ & $-20,0$ & $-14,0$ & $-10,0$ & $-15,0$ \\
\hline Max. & 25,0 & 15,0 & 31,0 & 13,0 & 11,0 & 10,0 & 10,0 & 18,0 & 19,0 \\
\hline \multicolumn{10}{|c|}{ Analyse der Gruppenunterschiede } \\
\hline \multicolumn{4}{|c|}{ Analyse der Zeitdifferenz } & \multicolumn{6}{|c|}{$\mathrm{p}=0,0047$ (LC); $\mathrm{p}=0,1359$ (LF); $\mathrm{p}=0,0175$ (LFRGL) } \\
\hline \multicolumn{4}{|l|}{ T1-T0 } & \multicolumn{6}{|c|}{$\mathrm{p}=0,0004(\mathrm{LC}) ; \mathrm{p}=0,0872$ (LF); $\mathrm{p}=0,0025$ (LFRGL) } \\
\hline \multicolumn{4}{|l|}{$\mathrm{T} 2-\mathrm{T} 1$} & \multicolumn{6}{|c|}{$\mathrm{p}=0,1290$ (LC); $\mathrm{p}=0,2039$ (LF); $\mathrm{p}=0,0130$ (LFRGL) } \\
\hline \multicolumn{4}{|l|}{ T2-T0 } & \multicolumn{6}{|c|}{$\mathrm{p}=0,1198$ (LC); $\mathrm{p}=0,4987$ (LF); $\mathrm{p}=0,6577$ (LFRGL) } \\
\hline \multicolumn{4}{|c|}{$\begin{array}{l}\text { Analyse der Wechselwirkung Gruppe } \\
\text { und Zeit }\end{array}$} & \multicolumn{6}{|c|}{$\mathrm{p}=0,0001$} \\
\hline
\end{tabular}

Tabelle 18: Änderung des HDL-Cholesterins

\subsubsection{Vergleich der Laborwerte}

Die Glucosewerte zeigen einen Trend zum Sinken zwischen T0 und T1 mit anschließendem Wiederanstieg. Dies ist in allen 3 Gruppen der Fall. Dabei sinkt der Glucosespiegel im Mittel in der Gruppe LC am stärksten ab mit 7,4 mg/dl. Der Wiederanstieg zwischen T1 und T2 fällt dort am geringsten aus, wodurch es bei einer Reduktion der Werte von T0 zu T2 bleibt. In den beiden Low-fat-Gruppen steigt der Glucosespiegel im Mittel von T1 zu T2 stär- 
ker an, als er von T0 zu T1 abfällt. Es kommt so zu einem Nettoanstieg der Glucosespiegel von T0 zu T2. Die Ergebnisse zwischen T0 und T2 sind in allen Gruppe recht gering und statistisch mit p=0,0946 (LC), p=0,1820 (LF) und p=0,0891 (LFRGL) nicht signifikant. Die Ergebnisse zeigen jedoch insgesamt einen signifikanten Unterschied in den Zeitverläufen zwischen der Gruppe LC und den beiden fettarmen Gruppen.

Bei den Triglyceriden ist nur in der Gruppe LC mit -42,7 mg/dl ein signifikanter Abfall der Werte über die Zeit zu erkennen $(\mathrm{p}=0,0022)$. In den beiden anderen Gruppen verfehlen die Ergebnisse das Signifikanzniveau. In der Gruppe LFRGL kommt es zu keinem Abfall der Werte von T0 zu T2, allerdings besteht in dieser Gruppe insgesamt ein deutlich geringerer Ausgangswert mit 117,5 mg/dl gegenüber 141,5 mg/dl und 141,8 mg/dl in LC und LF.

Auch die grafische Auftragung des Gesamtcholesterins zeigt einen ähnlichen Verlauf wie die Glucose und die Triglyceride mit Abfall zu T1 und Wiederanstieg der mittleren Werte zu T2, und das in allen Gruppen. Entsprechend gibt es keine signifikanten Gruppendifferenzen. Lediglich die Veränderung des Gesamtcholesterins über die Zeit ist in allen Gruppen signifikant, mit einer Reduktion zwischen T0 und T2 um durchschnittlich ca.-10 mg/dl in allen Gruppen. Für die LDL-Cholesterinwerte gibt es ebenfalls keine signifikanten Unterschiede zwischen den Gruppen, sondern nur signifikante Reduktionen über die Zeit. Dies gilt nicht für das HDL-Cholesterin. Hier findet man in keiner Gruppe eine signifikante Veränderung zwischen T0 und T2 (p>0,1 für alle Gruppen), dafür aber signifikante Unterschiede in den Zeitverläufen zwischen den Diätgruppen. Während in der Gruppe LC ein leichter Anstieg von $3,7 \mathrm{mg} / \mathrm{dl}$ zu verzeichnen ist, sinkt das HDL in den beiden Low-fat-Gruppen minimal ab.

\subsubsection{Harnsäure, Nieren- und Leberwerte}

\subsubsection{Messergebnisse}

\section{Harnsäure in mg/dl}

\begin{tabular}{|l|ccc|ccc|ccc|}
\hline & \multicolumn{2}{|c}{ Gruppe LC } & \multicolumn{3}{|c|}{ Gruppe LF } & \multicolumn{3}{c|}{ Gruppe LFRGL } \\
Zeitpunkt & T0 & T1 & T2 & T0 & T1 & T2 & T0 & T1 & T2 \\
\hline Mittel & 5,0 & 5,0 & 4,8 & 5,1 & 5,1 & 5,1 & 5,4 & 5,3 & 5,3 \\
Median & 5,0 & 5,1 & 4,8 & 5,3 & 5,1 & 5,2 & 5,5 & 5,3 & 5,4 \\
SD & 0,9 & 0,9 & 0,8 & 1,0 & 1,1 & 1,0 & 0,9 & 0,8 & 1,0 \\
Min. & 3,3 & 3,3 & 3,5 & 3,0 & 3,4 & 3,0 & 3,8 & 3,8 & 3,6 \\
Max. & 7,2 & 7,8 & 6,5 & 7,2 & 7,6 & 6,6 & 6,8 & 6,8 & 6,9 \\
\hline
\end{tabular}

Tabelle 19:Harnsäurewerte 
Kreatinin in $\mathbf{m g} / \mathbf{d l}$

\begin{tabular}{|l|ccc|ccc|ccc|}
\hline & \multicolumn{2}{|c}{ Gruppe LC } & \multicolumn{3}{|c|}{ Gruppe LF } & \multicolumn{3}{c|}{ Gruppe LFRGL } \\
Zeitpunkt & T0 & T1 & T2 & T0 & T1 & T2 & T0 & T1 & T2 \\
\hline Mittel & 0,8 & 0,8 & 0,7 & 0,8 & 0,8 & 0,7 & 0,8 & 0,9 & 0,8 \\
Median & 0,8 & 0,8 & 0,7 & 0,8 & 0,8 & 0,7 & 0,8 & 0,9 & 0,8 \\
SD & 0,1 & 0,1 & 0,1 & 0,1 & 0,1 & 0,1 & 0,1 & 0,1 & 0,1 \\
Min. & 0,7 & 0,7 & 0,5 & 0,7 & 0,7 & 0,6 & 0,6 & 0,6 & 0,6 \\
Max. & 1,1 & 1,1 & 1,0 & 1,0 & 1,0 & 1,0 & 1,1 & 1,1 & 1,2 \\
\hline
\end{tabular}

Tabelle 20: Kreatininwerte

Harnstoff in mg/dl

\begin{tabular}{|l|ccc|ccc|ccc|}
\hline & \multicolumn{3}{|l}{ Gruppe LC } & \multicolumn{4}{|l|}{ Gruppe LF } & \multicolumn{3}{|c|}{ Gruppe LFRGL } \\
Zeitpunkt & T0 & T1 & T2 & T0 & T1 & T2 & T0 & T1 & T2 \\
\hline Mittel & 14,3 & 15,2 & 15,2 & 13,8 & 13,0 & 12,6 & 12,5 & 12,5 & 13,4 \\
Median & 14,0 & 15,0 & 15,0 & 13,0 & 13,0 & 12,0 & 12,0 & 12,0 & 13,0 \\
SD & 3,3 & 3,2 & 3,5 & 3,7 & 2,8 & 2,8 & 2,7 & 3,0 & 3,5 \\
Min. & 9,0 & 10,0 & 9,0 & 9,0 & 8,0 & 7,0 & 8,0 & 7,0 & 9,0 \\
Max. & 20,0 & 24,0 & 22,0 & 22,0 & 19,0 & 19,0 & 19,0 & 20,0 & 25,0 \\
\hline
\end{tabular}

Tabelle 21:Harnstoffwerte

Aspartat-Aminotransferase (ASAT)/ Glutamat-Oxalacetat-Transaminase (GOT) in U/l

\begin{tabular}{|l|ccc|ccc|ccc|}
\hline & \multicolumn{3}{|l}{ Gruppe LC } & \multicolumn{4}{|l|}{ Gruppe LF } & \multicolumn{3}{|c|}{ Gruppe LFRGL } \\
Zeitpunkt & T0 & T1 & T2 & T0 & T1 & T2 & T0 & T1 & T2 \\
\hline Mittel & 22,3 & 24,2 & 21,7 & 23,4 & 23,3 & 23,3 & 22,9 & 23,6 & 22,5 \\
Median & 21,5 & 24,0 & 22,0 & 23,0 & 23,0 & 22,0 & 22,0 & 22,0 & 20,0 \\
SD & 4,3 & 4,9 & 4,6 & 5,8 & 5,8 & 4,8 & 6,6 & 7,7 & 6,8 \\
Min. & 17,0 & 13,0 & 16,0 & 14,0 & 12,0 & 12,0 & 13,0 & 11,0 & 15,0 \\
Max. & 34,0 & 32,0 & 38,0 & 38,0 & 40,0 & 34,0 & 41,0 & 50,0 & 50,0 \\
\hline
\end{tabular}

Tabelle 22: Veränderungen der ASAT/GOT

Alanin-Aminotransferase (ALAT)/ Glutamat-Pyruvat-Transaminase (GPT) in U/l

\begin{tabular}{|l|ccc|ccc|ccc|}
\hline & \multicolumn{3}{|l}{ Gruppe LC } & \multicolumn{3}{|c|}{ Gruppe LF } & \multicolumn{3}{|c|}{ Gruppe LFRGL } \\
Zeitpunkt & T0 & T1 & T2 & T0 & T1 & T2 & T0 & T1 & T2 \\
\hline Mittel & 21,2 & 24,8 & 17,3 & 22,3 & 21,6 & 16,6 & 23,4 & 24,4 & 19,9 \\
Median & 20,5 & 23,5 & 15,5 & 18,0 & 17,0 & 14,0 & 19,0 & 20,0 & 17,0 \\
SD & 7,5 & 10,7 & 7,1 & 13,5 & 12,2 & 6,5 & 11,9 & 13,8 & 13,0 \\
Min. & 10,0 & 12,0 & 6,0 & 8,0 & 10,0 & 7,0 & 11,0 & 11,0 & 7,0 \\
Max. & 38,0 & 60,0 & 31,0 & 60,0 & 65,0 & 34,0 & 61,0 & 76,0 & 76,0 \\
\hline
\end{tabular}

Tabelle 23: Veränderungen der ALAT/GPT 
Alkalische Phosphatase (AP) in U/I

\begin{tabular}{|l|ccc|ccc|ccc|}
\hline & \multicolumn{3}{|l}{ Gruppe LC } & \multicolumn{4}{c|}{ Gruppe LF } & \multicolumn{3}{c|}{ Gruppe LFRGL } \\
Zeitpunkt & T0 & T1 & T2 & T0 & T1 & T2 & T0 & T1 & T2 \\
\hline Mittel & 71,9 & 61,8 & 60,6 & 71,0 & 67,4 & 66,9 & 67,0 & 64,5 & 65,9 \\
Median & 72,0 & 61,0 & 58,0 & 68,0 & 68,0 & 64,0 & 60,0 & 61,0 & 60,0 \\
SD & 18,7 & 15,4 & 15,6 & 20,7 & 15,8 & 13,2 & 17,0 & 19,8 & 18,0 \\
Min. & 35,0 & 33,0 & 29,0 & 37,0 & 41,0 & 43,0 & 42,0 & 33,0 & 40,0 \\
Max. & 100,0 & 86,0 & 87,0 & 133,0 & 101,0 & 91,0 & 108,0 & 132,0 & 116,0 \\
\hline
\end{tabular}

Tabelle 24: Veränderungen der AP

$\gamma$-Glutamyl-Transferase (GGT) in U/l

\begin{tabular}{|l|ccc|ccc|ccc|}
\hline & \multicolumn{3}{|l}{ Gruppe LC } & \multicolumn{3}{|c|}{ Gruppe LF } & \multicolumn{3}{c|}{ Gruppe LFRGL } \\
Zeitpunkt & T0 & T1 & T2 & T0 & T1 & T2 & T0 & T1 & T2 \\
\hline Mittel & 27,3 & 17,3 & 19,7 & 18,6 & 15,4 & 16,7 & 19,7 & 17,4 & 20,5 \\
Median & 18,0 & 13,0 & 18,0 & 15,0 & 13,0 & 16,0 & 16,0 & 14,0 & 14,0 \\
SD & 23,3 & 15,8 & 9,9 & 10,3 & 7,5 & 7,3 & 12,2 & 11,0 & 14,2 \\
Min. & 6,0 & 5,0 & 7,0 & 6,0 & 3,0 & 6,0 & 8,0 & 8,0 & 8,0 \\
Max. & 116,0 & 79,0 & 50,0 & 46,0 & 38,0 & 43,0 & 64,0 & 52,0 & 66,0 \\
\hline
\end{tabular}

Tabelle 25: Veränderungen der GGT

\subsubsection{Vergleich der Messergebnisse}

Der mittlere Harnsäurewert liegt in allen Gruppen und zu allen Zeitpunkten innerhalb des Referenzbereichs von 2,6-5,3 mg/dl. In keiner Gruppe kommt es unter der Diät zu einer wesentlichen Veränderung. Ähnlich sieht die Situation bei den Kreatininwerten aus. Alle Gruppenmittelwerte liegen durchgehend im Normbereich von 0,5-0,9 mg/dl und die Veränderungen über die Zeit sind irrelevant klein. Bezüglich des Harnstoffs gibt es ebenfalls nur minimale Veränderungen innerhalb des mittleren Normbereichs von 8-21 mg/dl. Dabei erfolgt in Gruppe LF eine Senkung von -1,2 mg/dl, während es in den Gruppen LC und LFRGL zu einem kleinen Anstieg um 0,9 beziehungsweise 1,0 mg/dl kommt.

Für die Transaminasen Aspartat-Aminotransferase (ASAT)/ Glutamat-OxalacetatTransaminase (GOT), Alanin-Aminotransferase (ALAT)/ Glutamat-Pyruvat-Transaminase (GPT) und die $\gamma$-Glutamyl-Transferase (GGT) liegen die Mittelwerte ebenfalls allesamt im Normbereich, und es ergeben sich im Zeitverlauf unter den Diäten keine signifikanten Veränderungen. Nur für die Alkalische Phosphatase (AP) ist eine deutlichere Absenkung in der Gruppe LC von T0 zu T2 mit -11,3 U/l nachzuweisen. In den anderen beiden Gruppen sind dagegen nur unwesentliche Tendenzen zur Senkung um -4,1 (LF) und -1,1 U/l (LFRGL) zu erkennen. 


\subsection{Auswertung der Ernährungserfassung}

Die Erfassung der Nahrungsaufnahme erfolgte zum Zeitpunkt T0 vor Beginn und zum Zeitpunkt T2 nach 6 Monaten der Diätintervention. Aus den retrospektiven Angaben der Nahrungsaufnahme der einzelnen Teilnehmerinnen wurden mithilfe des MediTouch-Programms die mittleren Mengen für die tägliche Makro- und Mikronährstoffzufuhr berechnet.

\subsubsection{Kalorien, Makronährstoffe und ausgewählte Mikronährstoffe}

\subsubsection{Ergebnisse des MediTouch-Programms}

Kalorienaufnahme in kcal/Tag

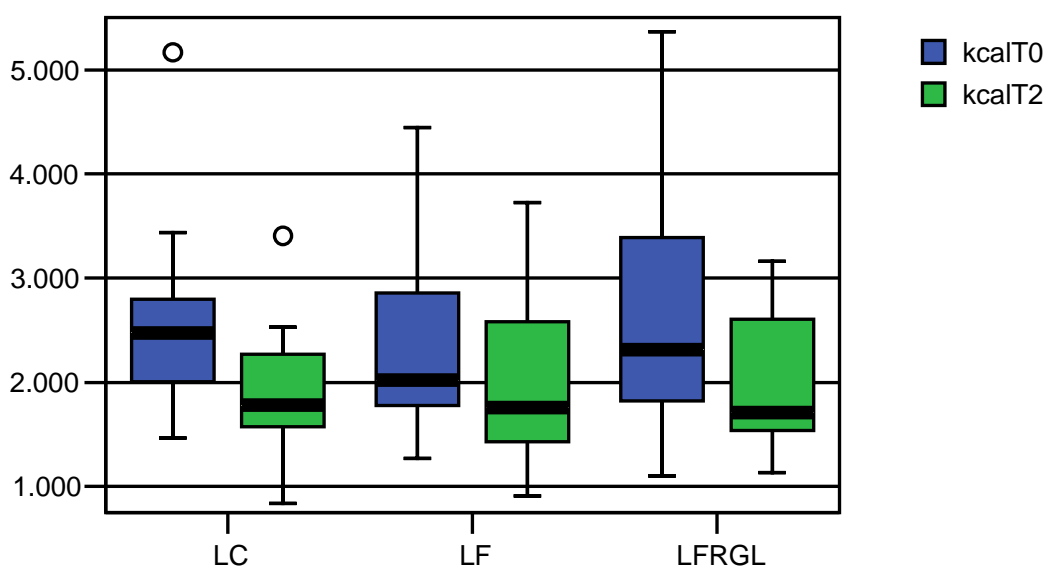

\begin{tabular}{|c|c|c|c|c|c|c|c|c|c|}
\hline \multirow[b]{2}{*}{ Zeitpunkt } & \multicolumn{3}{|c|}{ Gruppe LC } & \multicolumn{3}{|c|}{ Gruppe LF } & \multicolumn{3}{|c|}{ Gruppe LFRGL } \\
\hline & T0 & T2 & T2-T0 & T0 & T2 & T2-T0 & T0 & T2 & T2-T0 \\
\hline Mittel & 2527,0 & 1875,3 & $-651,8$ & 2395,2 & 2000,3 & $-394,9$ & 2573,8 & 1981,2 & $-592,6$ \\
\hline Median & 2476,1 & 1782,4 & $-581,9$ & 2023,3 & 1758,0 & $-275,4$ & 2312,5 & 1709,9 & $-487,1$ \\
\hline SD & 766,3 & 581,2 & 737,5 & 913,2 & 748,2 & 707,4 & 1026,0 & 604,0 & 712,7 \\
\hline Min. & 1463,5 & 840,1 & $-1920,4$ & 1267,8 & 910,0 & $-2394,4$ & 1102,5 & 1133,9 & $-2813,9$ \\
\hline Max. & 5167,0 & 3406,2 & 713,4 & 4447,0 & 3725,4 & 764,2 & 5363,9 & 3162,7 & 725,5 \\
\hline \multicolumn{4}{|c|}{ Analyse der Gruppenunterschiede } & \multicolumn{6}{|c|}{$p=0,8864$} \\
\hline \multicolumn{4}{|c|}{ Analyse der Zeitdifferenz } & \multicolumn{6}{|c|}{$p<0,0001$} \\
\hline \multirow{2}{*}{\multicolumn{4}{|c|}{$\begin{array}{l}\text { Analyse der Wechselwirkung Gruppe } \\
\text { und Zeit }\end{array}$}} & \multirow{2}{*}{\multicolumn{3}{|c|}{$\mathrm{p}=0,3780$}} & & & \\
\hline & & & & & & & & & \\
\hline
\end{tabular}

Tabelle 26: Veränderung des Kalorienverzehrs 


\begin{tabular}{|c|c|c|c|c|c|c|c|c|c|}
\hline \multirow[b]{2}{*}{ Zeitpunkt } & \multicolumn{3}{|c|}{ Gruppe LC } & \multicolumn{3}{|c|}{ Gruppe LF } & \multicolumn{3}{|c|}{ Gruppe LFRGL } \\
\hline & T0 & $\mathrm{T} 2$ & T2-T0 & T0 & T2 & T2-T0 & T0 & $\mathrm{T} 2$ & T2-T0 \\
\hline Mittel & 93,6 & 98,2 & 4,5 & 88,7 & 45,8 & $-42,9$ & 92,6 & 55,6 & $-37,0$ \\
\hline Median & 88,3 & 88,1 & $-3,0$ & 72,4 & 43,5 & $-29,9$ & 89,6 & 48,6 & $-30,3$ \\
\hline SD & 40,7 & 38,6 & 49,5 & 44,5 & 21,0 & 33,0 & 45,1 & 19,8 & 35,6 \\
\hline Min. & 45,0 & 23,0 & $-73,1$ & 31,2 & 10,6 & $-112,9$ & 24,6 & 20,8 & $-129,0$ \\
\hline Max. & 218,7 & 173,6 & 116,9 & 183,9 & 83,0 & $-1,7$ & 216,4 & 106,3 & 3,4 \\
\hline \multicolumn{4}{|c|}{ Analyse der Gruppenunterschiede } & \multicolumn{6}{|c|}{$p=0,0002$} \\
\hline \multicolumn{4}{|c|}{ Analyse der Zeitdiffere } & \multicolumn{6}{|c|}{$\mathrm{p}=0,5725$ (LC); $\mathrm{p}<0,0001$ (LF); $\mathrm{p}<0,0001$ (LFRGL) } \\
\hline \multicolumn{4}{|c|}{$\begin{array}{l}\text { Analyse der Wechselwirkung Gruppe } \\
\text { und Zeit }\end{array}$} & \multicolumn{6}{|c|}{$p<0,0001$} \\
\hline
\end{tabular}

Tabelle 28: Veränderungen des Fettverzehrs

\section{Eiweißaufnahme in g/Tag}

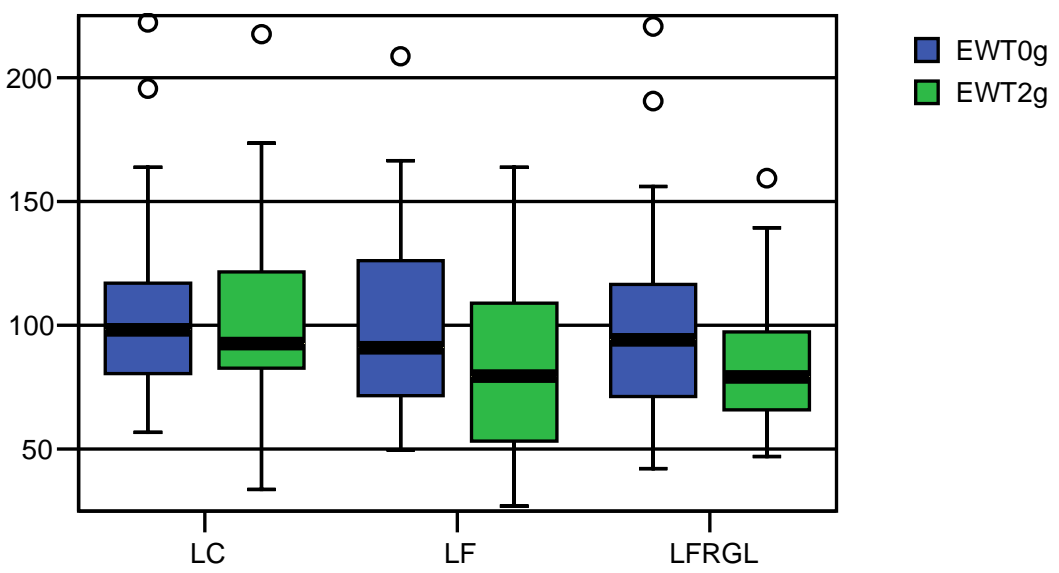

\begin{tabular}{|c|c|c|c|c|c|c|c|c|c|}
\hline \multirow[b]{2}{*}{ Zeitpunkt } & \multicolumn{3}{|c|}{ Gruppe LC } & \multicolumn{3}{|c|}{ Gruppe LF } & \multicolumn{3}{|c|}{ Gruppe LFRGL } \\
\hline & T0 & T2 & T2-T0 & T0 & T2 & T2-T0 & T0 & T2 & T2-T0 \\
\hline Mittel & 108,2 & 101,6 & $-6,6$ & 102,5 & 83,0 & $-19,5$ & 98,9 & 85,3 & $-13,5$ \\
\hline Median & 98,2 & 92.5 & -7.7 & 91.0 & 79.5 & -16.9 & 94.2 & 79,2 & $-10,2$ \\
\hline SD & 40,6 & 37,9 & 38,6 & 42,5 & 37,0 & 28,3 & 42,4 & 27,6 & 30,9 \\
\hline Min. & 56,7 & 33,7 & $-83,3$ & 49,7 & 27,0 & $-91,6$ & 42,1 & 47,0 & $-99,4$ \\
\hline Max. & 222,3 & 217,6 & 71,6 & 208,7 & 163,8 & 37,1 & 220,7 & 159,4 & 60,7 \\
\hline \multicolumn{4}{|c|}{ Analyse der Gruppenunterschiede } & \multicolumn{3}{|c|}{$p=0,3023$} & & & \\
\hline \multicolumn{4}{|c|}{ Analyse der Zeitdifferenz } & \multicolumn{3}{|c|}{$p=0,0003$} & & & \\
\hline \multirow{2}{*}{\multicolumn{4}{|c|}{$\begin{array}{l}\text { Analyse der Wechselwirkung Gruppe } \\
\text { und Zeit }\end{array}$}} & \multirow{2}{*}{\multicolumn{3}{|c|}{$p=0,3279$}} & & & \\
\hline & & & & & & & & & \\
\hline
\end{tabular}

Tabelle 29: Veränderungen des Eiweißverzehrs 
Aufnahme von Ballaststoffen in g/Tag

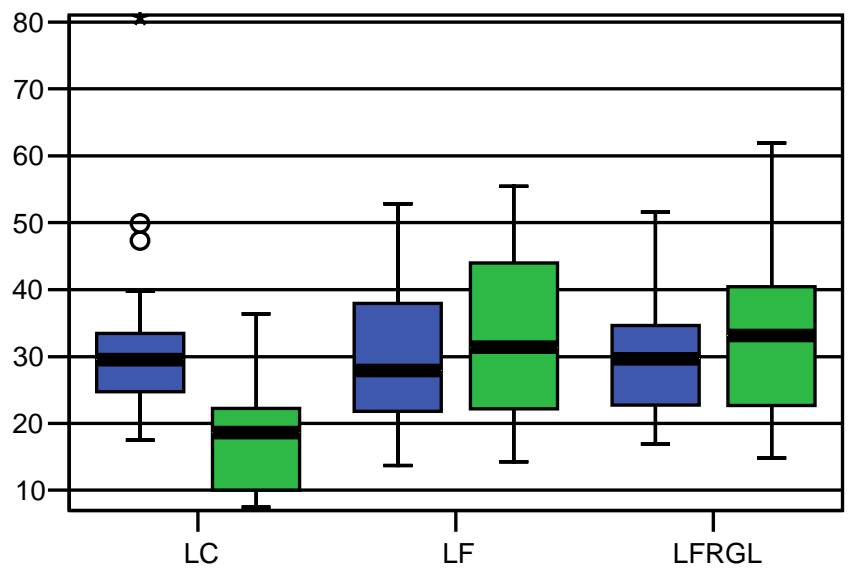

\begin{tabular}{|l|cc|c|cc|c|cc|c|}
\hline & \multicolumn{4}{l}{ Gruppe LC } & \multicolumn{2}{l|}{ Gruppe LF } & \multicolumn{2}{l|}{ Gruppe LFRGL } \\
\cline { 2 - 10 } Zeitpunkt & T0 & T2 & T2-T0 & T0 & T2 & T2-T0 & T0 & T2 & T2-T0 \\
\hline Mittel & 31,7 & 18,0 & $-13,8$ & 30,3 & 32,9 & 2,7 & 29,9 & 33,7 & 3,8 \\
Median & 29,5 & 18,6 & $-13,4$ & 27,9 & 31,4 & 2,8 & 29,7 & 33,1 & 3,9 \\
SD & 12,6 & 8,1 & 12,6 & 11,0 & 12,5 & 12,2 & 9,6 & 13,3 & 10,6 \\
Min. & 17,5 & 7,5 & $-61,0$ & 13,7 & 14,3 & $-24,0$ & 17,0 & 14,9 & $-13,8$ \\
Max. & 80,6 & 36,4 & 5,0 & 52,8 & 55,5 & 33,7 & 51,5 & 61,9 & 30,8 \\
\hline Analyse der Gruppenunterschiede & \multicolumn{10}{|c|}{$\mathrm{p}=0,0117$} \\
Analyse der Zeitdifferenz & $\mathrm{p}<0,0001$ (LC); $\mathrm{p}=0,2659$ (LF); $\mathrm{p}=0,0459$ (LFRGL) \\
Analyse der Wechselwirkung Gruppe \\
und Zeit
\end{tabular}

Tabelle 30: Änderung des Verzehrs von Ballaststoffen

\section{Aufnahme von gesättigten Fettsäuren (GFS) in g/Tag}

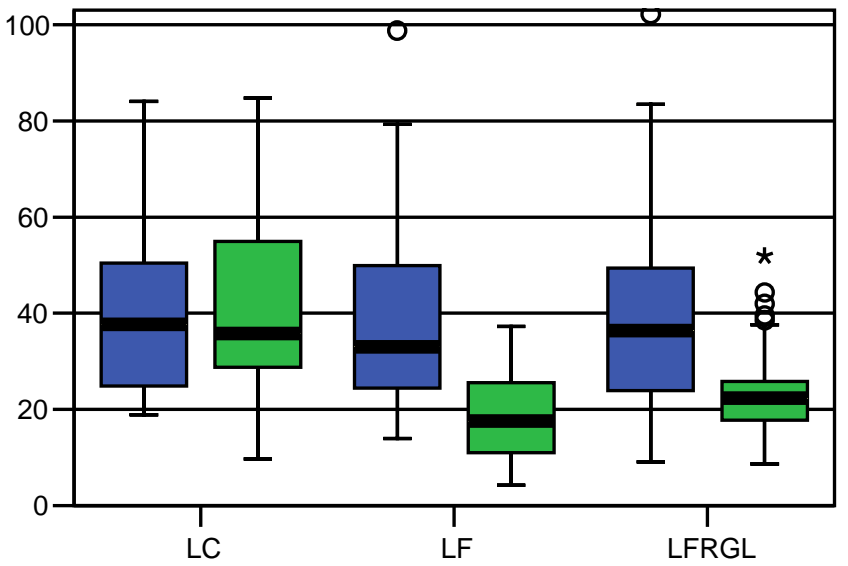




\begin{tabular}{|c|c|c|c|c|c|c|c|c|c|}
\hline \multirow[b]{2}{*}{ Zeitpunkt } & \multicolumn{3}{|c|}{ Gruppe LC } & \multicolumn{3}{|c|}{ Gruppe LF } & \multicolumn{3}{|c|}{ Gruppe LFRGL } \\
\hline & T0 & T2 & T2-T0 & T0 & T2 & T2-T0 & T0 & $\mathrm{T} 2$ & T2-T0 \\
\hline Mittel & 39,8 & 42,1 & 2,3 & 39,8 & 19,0 & $-20,8$ & 40,9 & 24,0 & $-16,9$ \\
\hline Median & 37,7 & 35,8 & 0,5 & 33,0 & 17,6 & $-15,8$ & 36,4 & 22,4 & $-11,7$ \\
\hline SD & 17,0 & 19,0 & 22,7 & 22,2 & 9,3 & 16,7 & 21,7 & 10,3 & 17,7 \\
\hline Min. & 18,9 & 9,7 & $-36,1$ & 14,0 & 4,2 & $-61,5$ & 9,1 & 8,7 & $-63,8$ \\
\hline Max. & 84,1 & 84,8 & 61,5 & 98,8 & 37,3 & 1,1 & 102,2 & 51,9 & 2,2 \\
\hline \multicolumn{4}{|c|}{ Analyse der Gruppenunterschiede } & \multicolumn{6}{|c|}{$p=0,0007$} \\
\hline \multicolumn{4}{|c|}{ Analyse der Zeitdiffe } & \multicolumn{6}{|c|}{$\mathrm{p}=0,6933$ (LC); $\mathrm{p}<0,0001$ (LF); $\mathrm{p}<0,0001$ (LFRGL) } \\
\hline \multicolumn{4}{|c|}{$\begin{array}{l}\text { Analyse der Wechselwirkung Gruppe } \\
\text { und Zeit }\end{array}$} & \multicolumn{6}{|c|}{$p<0,0001$} \\
\hline
\end{tabular}

Tabelle 31: Änderung des Verzehrs gesättigter Fettsäuren

\section{Aufnahme von einfach ungesättigten Fettsäuren (EUFS) in g/Tag}

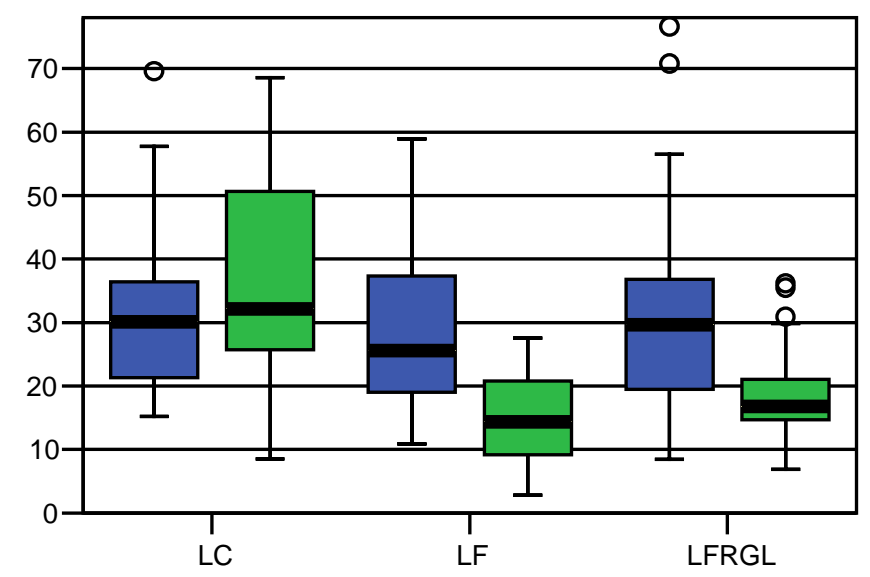

\begin{tabular}{|c|c|c|c|c|c|c|c|c|c|}
\hline \multirow[b]{2}{*}{ Zeitpunkt } & \multicolumn{3}{|c|}{ Gruppe LC } & \multicolumn{3}{|c|}{ Gruppe LF } & \multicolumn{3}{|c|}{ Gruppe LFRGL } \\
\hline & T0 & $\mathrm{T} 2$ & T2-T0 & TO & T2 & T2-T0 & T0 & $\mathrm{T} 2$ & T2-T0 \\
\hline Mittel & 32,3 & 36,5 & 4,1 & 30,3 & 15,1 & $-15,2$ & 31,5 & 18,5 & $-12,9$ \\
\hline Median & 30,1 & 32,2 & 0,7 & 25,6 & 14,4 & $-11,1$ & 29,6 & 16,8 & $-11,5$ \\
\hline SD & 14,7 & 15,0 & 18,5 & 15,5 & 7,4 & 12,1 & 16,3 & 6,9 & 13,1 \\
\hline Min. & 15,2 & 8,5 & $-25,6$ & 10,9 & 2,8 & $-42,4$ & 8,5 & 6,9 & $-50,2$ \\
\hline Max. & 69,6 & 68,6 & 42,3 & 58,9 & 27,6 & $-1,3$ & 76,6 & 36,2 & 1,3 \\
\hline \multirow{3}{*}{\multicolumn{4}{|c|}{$\begin{array}{l}\text { Analyse der Gruppenunterschiede } \\
\text { Analyse der Zeitdifferenz } \\
\text { Analyse der Wechselwirkung Gruppe } \\
\text { und Zeit }\end{array}$}} & \multicolumn{6}{|c|}{$p<0,0001$} \\
\hline & & & & \multicolumn{6}{|c|}{$p=0,1969$ (LC); $p<0,0001$ (LF); $p<0,0001$ (LFRGL) } \\
\hline & & & & \multicolumn{6}{|c|}{$p<0,0001$} \\
\hline
\end{tabular}

Tabelle 32: Änderung des Verzehrs einfach ungesättigter Fettsäuren 


\begin{tabular}{|c|c|c|c|c|c|c|c|c|c|}
\hline \multirow[b]{2}{*}{ Zeitpunkt } & \multicolumn{3}{|c|}{ Gruppe LC } & \multicolumn{3}{|c|}{ Gruppe LF } & \multicolumn{3}{|c|}{ Gruppe LFRGL } \\
\hline & T0 & $\mathrm{T} 2$ & T2-T0 & T0 & $\mathrm{T} 2$ & T2-T0 & T0 & $\mathrm{T} 2$ & T2-T0 \\
\hline Mittel & 2,2 & 1,8 & $-0,5$ & 2,1 & 2,0 & $-0,1$ & 2,1 & 1,8 & $-0,3$ \\
\hline Median & 2,2 & 1,6 & $-0,5$ & 1,8 & 1,9 & $-0,2$ & 1,8 & 1,6 & $-0,2$ \\
\hline SD & 0,8 & 0,6 & 0,7 & 0,9 & 0,7 & 0,7 & 1,0 & 0,7 & 0,6 \\
\hline Min. & 1,0 & 1,0 & $-2,0$ & 0,9 & 0,7 & $-1,2$ & 0,8 & 0,9 & $-2,2$ \\
\hline Max. & 3,9 & 3,5 & 0,8 & 4,6 & 3,4 & 1,4 & 5,8 & 3,9 & 0,7 \\
\hline \multicolumn{4}{|c|}{ Analyse der Gruppenunterschiede } & \multicolumn{6}{|c|}{$p=0,5124$} \\
\hline \multicolumn{4}{|c|}{ Analyse der Zeitdiffer } & \multicolumn{6}{|c|}{$\mathrm{p}=0,0029$ (LC); $\mathrm{p}=0,9521$ (LF); $\mathrm{p}=0,0230$ (LFRGL) } \\
\hline \multicolumn{4}{|c|}{$\begin{array}{l}\text { Analyse der Wechselwirkung Gruppe } \\
\text { und Zeit }\end{array}$} & \multicolumn{6}{|c|}{$p=0,04565$} \\
\hline
\end{tabular}

Tabelle 34: Änderung der Aufnahme von Vitamin B6

\section{Vitamin-B12-Aufnahme in $\mu \mathrm{g} / \mathrm{Tag}$}

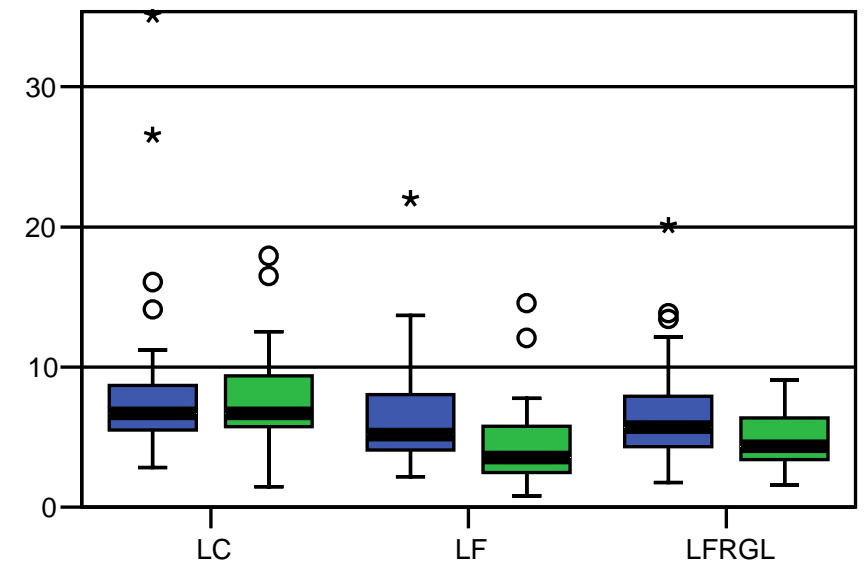

\begin{tabular}{|c|c|c|c|c|c|c|c|c|c|}
\hline \multirow[b]{2}{*}{ Zeitpunkt } & \multicolumn{3}{|c|}{ Gruppe LC } & \multicolumn{3}{|c|}{ Gruppe LF } & \multicolumn{3}{|c|}{ Gruppe LFRGL } \\
\hline & T0 & T2 & T2-T0 & T0 & $\mathrm{T} 2$ & T2-T0 & T0 & T2 & T2-T0 \\
\hline Mittel & 9,0 & 7,7 & $-1,3$ & 6,9 & 4,5 & $-2,5$ & 6,7 & 4,7 & $-2,0$ \\
\hline Median & 6,7 & 6,7 & $-0,2$ & 5,2 & 3,6 & $-2,2$ & 5,7 & 4,3 & $-0,8$ \\
\hline SD & 7,2 & 3,7 & 6,3 & 4,5 & 3,2 & 3,3 & 4,1 & 1,9 & 3,4 \\
\hline Min. & 2,8 & 1,5 & $-22,0$ & 2,2 & 0,8 & $-9,9$ & 1,8 & 1,6 & $-11,0$ \\
\hline Max. & 35,1 & 17,9 & 7,3 & 22,0 & 14,6 & 6,4 & 20,1 & 9,1 & 2,0 \\
\hline \multicolumn{4}{|c|}{$\begin{array}{l}\text { Analyse der Gruppenunterschiede } \\
\text { Analyse der Zeitdifferenz } \\
\text { Analyse der Wechselwirkung Gruppe } \\
\text { und Zeit }\end{array}$} & & $\begin{array}{l}09 \\
40 \text { (LC } \\
62\end{array}$ & $<0,0001$ & $(L F) ; p=0$ & (LFF & \\
\hline
\end{tabular}

Tabelle 35: Änderung der Aufnahme von Vitamin B12 
Folsäureaufnahme in $\mu \mathrm{g} / \mathrm{Tag}$

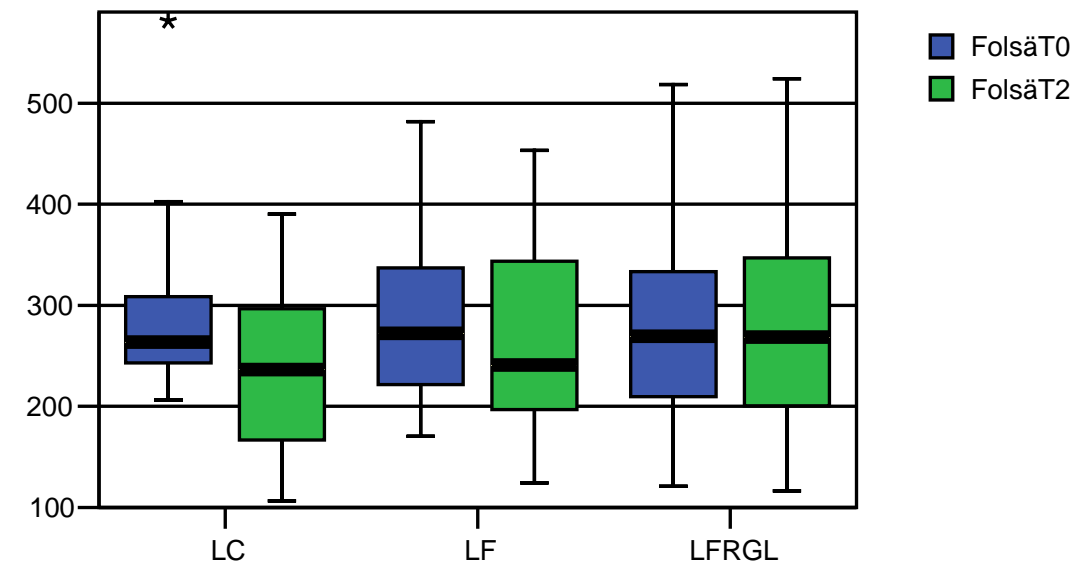

\begin{tabular}{|l|cc|c|cc|c|cc|c|}
\hline & \multicolumn{3}{l}{ Gruppe LC } & \multicolumn{2}{l|}{ Gruppe LF } & \multicolumn{2}{l|}{ Gruppe LFRGL } \\
\cline { 2 - 10 } Zeitpunkt & T0 & T2 & T2-T0 & T0 & T2 & T2-T0 & T0 & T2 & T2-T0 \\
\hline Mittel & 290,4 & 234,2 & $-56,2$ & 292,6 & 273,5 & $-19,1$ & 277,7 & 284,0 & 6,3 \\
Median & 263,7 & 236,3 & $-57,4$ & 272,1 & 240,9 & $-18,9$ & 269,3 & 268,5 & 11,0 \\
SD & 79,7 & 81,9 & 99,0 & 91,3 & 92,5 & 85,0 & 101,9 & 108,1 & 70,1 \\
Min. & 206,1 & 106,6 & $-297,6$ & 170,4 & 124,0 & $-273,3$ & 121,1 & 116,4 & $-130,3$ \\
Max. & 581,6 & 390,0 & 143,9 & 481,8 & 453,3 & 154,2 & 518,7 & 524,4 & 176,2 \\
\hline Analyse der Gruppenunterschiede & \multicolumn{10}{|c|}{$\mathrm{p}=0,5776$} \\
Analyse der Zeitdifferenz & $\mathrm{p}=0,0038$ (LC); $\mathrm{p}=0,2419$ (LF); $\mathrm{p}=0,6170$ (LFRGL) \\
Analyse der Wechselwirkung Gruppe & $\mathrm{p}=0,0272$ \\
und Zeit
\end{tabular}

Tabelle 36: Änderung der Aufnahme von Folsäure

\subsubsection{Vergleich der Nährstoffaufnahme}

Eindeutig ist die hoch signifikante Verminderung der Kalorienaufnahme in allen 3 Diätgruppen ( $\mathrm{p}<0,0001)$ mit -651,8 kcal (LC), -394,9 kcal (LF) und -592,6 kcal (LFRGL) im Zeitraum T0 bis T2. Und sie geschieht in allen Gruppen ohne Kalorienrestriktion bei den Diätvorgaben. Der Vergleich zeigt dabei nur geringe Unterschiede zwischen den Gruppen, die das Signifikanzniveau verfehlen. Eingespart wurden die Kalorien aber mit unterschiedlichen Makronährstoffen: Die Teilnehmerinnen in der Gruppe LC nahmen hoch signifikant weniger Kohlenhydrate mit -188,1 g/Tag zu sich (p<0,0001). Und in den beiden Low-fat-Gruppen reduzierten die Teilnehmerinnen hoch signifikant die Fettzufuhr $(\mathrm{p}<0,0001)$. Den größten Fetteinspareffekt erzielten die Probandinnen der Gruppe LF mit -42,9 g/Tag. In der Gruppe LFRGL findet man interessanterweise zusätzlich eine signifikante Verringerung des Kohlenhydratverzehrs von -56,2 g/Tag ( $\mathrm{p}=0,0175$ ). Dagegen fällt der Mehrverzehr von Kohlenhydraten in der Gruppe LF und der von Fetten in der Gruppe LC statistisch nicht signifikant aus. Alle 3 Gruppen haben zudem die Zufuhr von Eiweiß eingeschränkt. Der 
Minderverzehr fällt ohne diesbezügliche Diätanweisungen sogar signifikant aus. Das gilt für alle Gruppen gleichermaßen. Die Varianzanalyse fand weder deutliche Gruppenunterschiede noch unterschiedliche Zeitverläufe des Eiweißminderverzehrs.

Die prozentuale Verteilung und Änderung der Kalorienanteile für die unterschiedlichen Makronähstoffe zwischen den Zeitpunkten T0 und T2 läßt sich sehr anschaulich in Kreisdiagrammen für jede Gruppe darstellen.

\section{Prozentuale Kalorienverteilung der verzehrten Makronährstoffe T0}

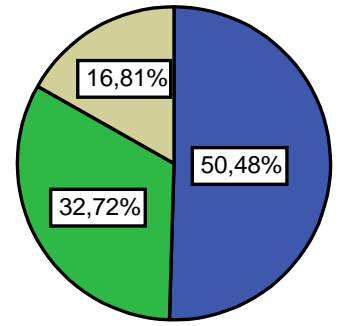

LC

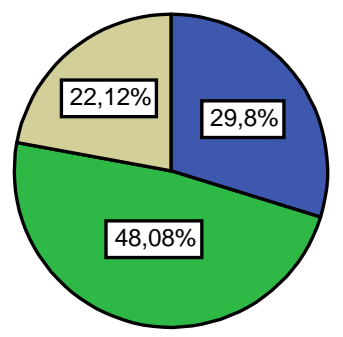

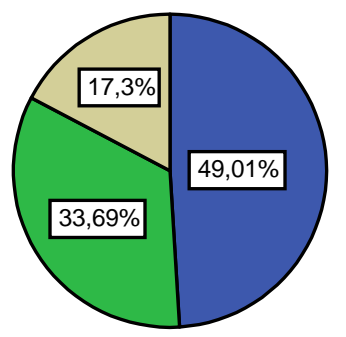

LF

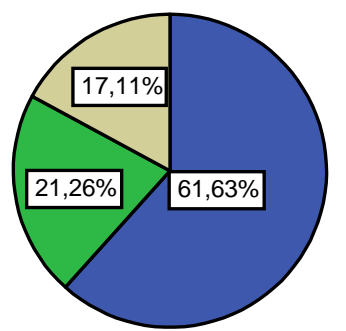

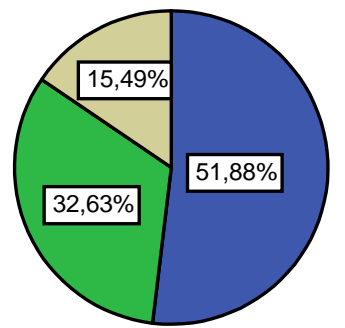

KHkcal

T0

Fekcal

T0

$\square$ EWkca

\section{LFRGL}

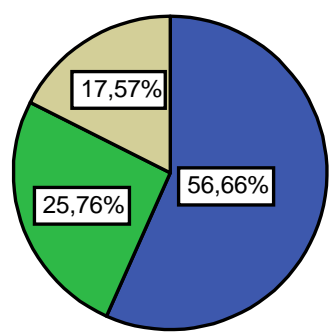

$\square$ KHkcal

T2

$\square$ Fekcal

T2

$\square$ EWkca

\section{Prozentuale Kalorienverteilung der verzehrten Makronährstoffe T2}

Abbildung 4: Vergleich der prozentualen Kalorienverteilung T0 und T2

Anhand der Grafik sieht man die Veränderungen der Makronährstoff-Kalorien zwischen den Zeitpunkten T0 und T2. In Gruppe LC geht der Anteil der Kohlenhydrat-Kalorien um über 20\% auf nur noch 29,8\% zurück und der Fettanteil steigt um 15,4\% auf 48,1\%. Dagegen nimmt der Kalorienanteil der Fette in der Gruppe LF prozentual um über 12\% ab. Die EiweißKalorien bleiben hier trotz Abnahme des Eiweißverzehrs insgesamt unverändert, während der Kalorienanteil der Kohlenhydrate um 12\% Prozent zunimmt. Die Veränderungen in der Gruppe LFRGL fallen etwas geringer aus. Der Fettanteil fällt mit ca. 7\% leichter ab, und bei geringem Anstieg der prozentualen Eiweiß-Kalorien steigt der Kohlenhydratanteil um 5\% auf $56,7 \%$. 
Die Ergebnisse des Ballaststoffverzehrs entsprechen vor dem Diätbeginn den Empfehlungen der DGE mit ca. 30 g/Tag. Unter der geforderten Reduktion der Kohlenhydrate auf maximal 60 g/Tag in der zweiten Diätphase der Gruppe LC fällt ein drastischer Minderverzehr von Ballaststoffen auf. Im Mittel nahmen die Teilnehmerinnen nur noch $18 \mathrm{~g} / \mathrm{Tag}$ zum Zeitpunkt T2 auf. Das entspricht einer sehr signifikanten Reduktion um fast 44\%. Bei freier Kohlenhydratzufuhr und Beschränkung des Fettverzehrs konnte in beiden Low-fat-Gruppen dagegen der Ballaststoffgehalt der Nahrung leicht gesteigert werden. Das Ergebnis entspricht allerdings lediglich für die Gruppe LFRGL einer signifikanten Veränderung gegenüber dem Ausgangswert $(\mathrm{p}=0,0459)$.

Der Gesamtfettverzehr in der Gruppe LC steigt trotz unbeschränkt erlaubter Fettaufnahme nur minimal, während er in den beiden Gruppen mit diätetischer Fettbeschränkung hoch signifikant zurückgeht. Dasselbe Ergebnis erhält man entsprechend auch für die Veränderungen der einzelnen Fettsäuren: Der Verzehr von gesättigten Fettsäuren steigt dabei in Gruppe LC weniger an als der von einfach ungesättigten Fettsäuren. Der Anteil von mehrfach ungesättigten Fettsäuren erhöht sich am geringsten. Dem entgegen geht der Verzehr von gesättigten und einfach ungesättigten Fettsäuren in den Low-fat-Gruppen jeweils ungefähr gleich stark zurück, in der Gruppe LF stärker als in LFRGL. Nur die mehrfach ungesättigten Fettsäuren werden in der Gruppe LFRGL stärker reduziert als in der Gruppe LF.

Die aus den Verzehrdaten ermittelten Werte für die Mikronährstoffe Folsäure, Vitamin B6 und B12 belegen, dass die empfohlenen Referenzwerte der täglichen Aufnahme für Vitamin B6 und B12 in allen 3 Gruppen und zu beiden Zeitpunkten T0 und T2 erreicht werden, allerdings für Folsäure nie. Die Referenzwerte für Vitamin B6 liegen zwischen 1,2-1,6 mg/Tag und für Vitamin B12 bei $3 \mu \mathrm{g} / \mathrm{Tag}$. Die empfohlene Mindestmenge von $400 \mu \mathrm{g}$ Folsäure/Tag erscheint verglichen mit dem tatsächlichen Verzehr der Probandinnen von weniger als $300 \mu \mathrm{g} / \mathrm{Tag}$ fast unerreichbar. Die Aufnahme sinkt ebenso wie die von Vitamin B6 unter kohlenhydratarmer Ernährung noch weiter ab. Unter der kohlenhydratarmen Ernährung beträgt die tägliche Aufnahme von Folsäure mit durchschnittlich $234 \mu$ g lediglich knapp 60\% der Tagesempfehlung der DGE. In den übrigen Gruppen sind keine signifikanten Änderungen zwischen den beiden Zeitpunkten weder für die Folsäureaufnahme noch für die Versorgung mit Vitamin B6 zu erkennen. Vitamin B12 wird in der Low-carb-Gruppe zu beiden Zeitpunkten in gleichem Maß aufgenommen. Bei fettarmer Ernährung vermindert sich der VitaminB12-Verzehr bei dennoch erreichter Referenzmenge. 


\subsubsection{Analyse der Compliance anhand des Makronährstoffverzehrs}

Eine Beurteilung der Compliance der Teilnehmerinnen anhand des Kriteriums, ob sie in der kohlenhydratarmen Gruppe die Grenze von maximal $60 \mathrm{~g}$ Kohlenhydraten pro Tag und in den fettarmen Gruppen weniger als $45 \mathrm{~g}$ Fett pro Tag verzehrt haben, ergibt v. a. für die erste Gruppe schlechte Compliancewerte. Nur 2 der 26 Frauen hatten die Kohlenhydratbeschränkung eingehalten. Auch die Teilnehmerinnen der Low-fat-Gruppen hatten nur teilweise den Vorgaben zur Fettbeschränkung streng Folge geleistet. Da wir den glykämischen Index der Nahrung mit dem MediTouch-Programm nicht ermittelt haben, kann man lediglich anhand der vermehrten Ballaststoffaufnahme eine Tendenz zur Compliance diesbezüglich annehmen. 7 von 8 Probandinnen der Gruppe LFRGL mit Compliance bezüglich der Fettaufnahme wiesen gleichzeitig wenigstens eine geringe Steigerung der Ballaststoffaufnahme auf.

Wenn nun die Gewichtsabnahme der Teilnehmerinnen, die das Kriterium eingehalten haben, gegen die Gewichtsänderung derjenigen, die es verfehlt haben, betrachtet wird, sind keine signifikanten Unterschiede erkennbar.

Gegenüberstellung der Gewichtsdifferenz T2-T0 in kg nach Compliancekriterien

\begin{tabular}{|l|c|c|c|c|c|c|}
\hline \multicolumn{3}{|c|}{} & \multicolumn{3}{|c|}{ Gruppe LC } & \multicolumn{2}{c|}{ Gruppe LF } & \multicolumn{2}{c|}{ Gruppe LFRGL } \\
Kriterium & $<60 \mathrm{~g} \mathrm{KH}$ & $>60 \mathrm{~g} \mathrm{KH}$ & $<45 \mathrm{~g}$ Fett & $>45 \mathrm{~g}$ Fett & $45 \mathrm{~g}$ Fett & $>45 \mathrm{~g}$ Fett \\
\hline Mittel & $-9,2$ & $-8,0$ & $-6,5$ & $-6,1$ & $-7,0$ & $-6,8$ \\
Median & $-9,2$ & $-7,9$ & $-6,9$ & $-6,0$ & $-7,2$ & $-6,9$ \\
SD & 2,0 & 4,1 & 4,4 & 3,4 & 3,9 & 3,5 \\
Min. & $-10,6$ & $-17,0$ & $-15,4$ & $-13,0$ & $-12,8$ & $-13,8$ \\
Max. & $-7,8$ & $-2,1$ & 0,0 & $-1,3$ & $-0,9$ & 0,1 \\
\hline Anzahl & $\mathbf{2}$ & $\mathbf{2 4}$ & $\mathbf{1 5}$ & $\mathbf{1 2}$ & $\mathbf{8}$ & $\mathbf{2 3}$ \\
\hline
\end{tabular}

Tabelle 37: Gewichtsdifferenz bei unterschiedlicher Compliance

\subsection{Korrelationsanalysen für die Körpermesswerte, Labor- und Verzehrdaten}

\subsubsection{Homocystein}

\subsubsection{Homocystein und Methylen-Tetrahydrofolatreduktase (MTHFR)}

Bei den 29 Teilnehmerinnen, von denen der Genotyp der MTHFR bestimmt wurde, zeigt sich in einer Auftragung des Genotyps gegenüber den Homocysteinwerten der T1Blutuntersuchung kein Zusammenhang zwischen diesen beiden Parametern. Der berechnete 
Spearman-Rangkorrelationskoeffizient ist $\mathrm{r}=-0,182(\mathrm{p}=0,344)$ und belegt auch mathematisch die fehlende Abhängigkeit.
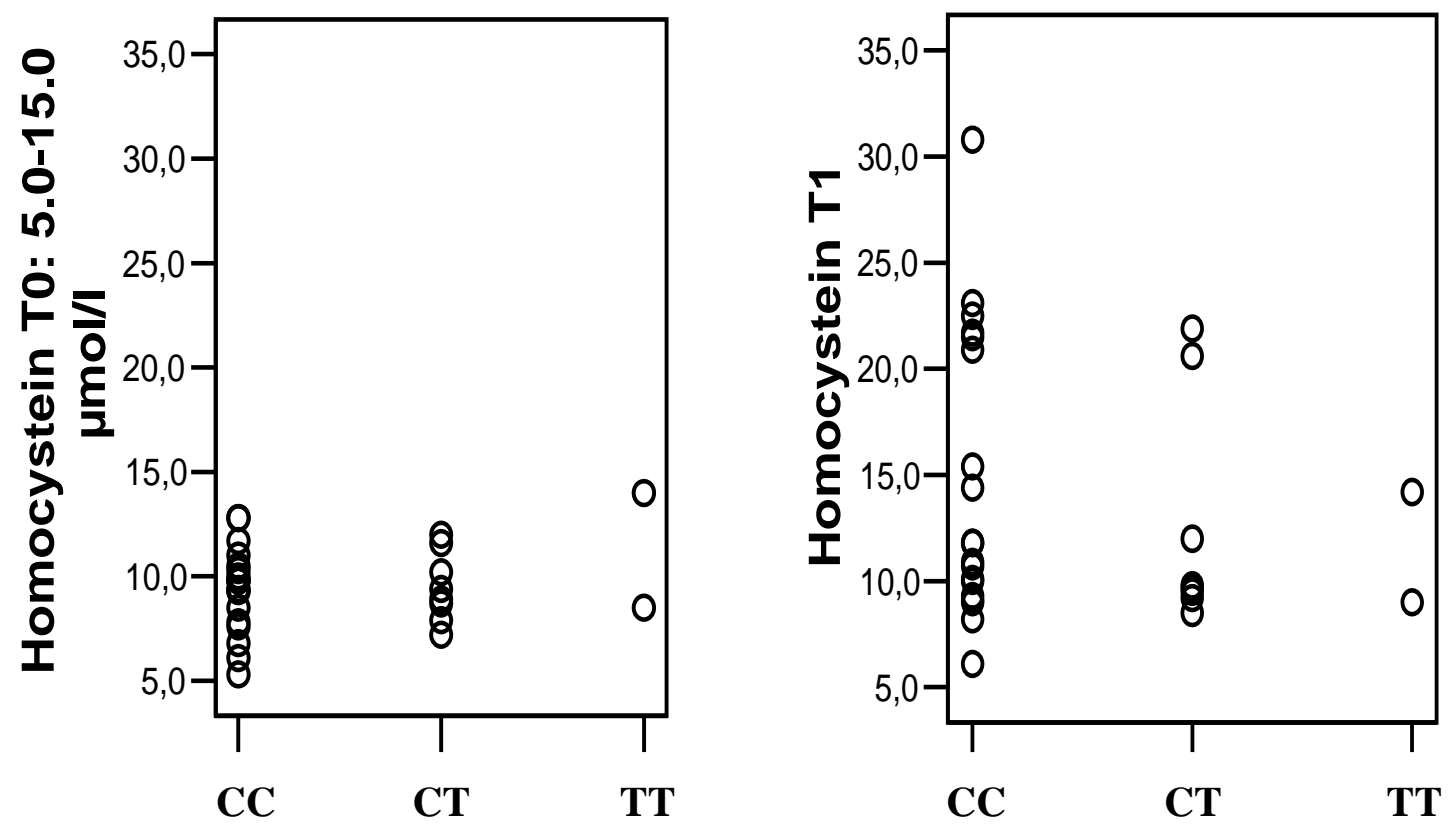

Abbildung 5: Homocysteinwerte T0 und T1 in Abhängigkeit vom MTHFR-Genotyp

\subsubsection{Homocystein, Körpermesswerte und andere Labordaten}

Weder für das Körpergewicht, den BMI oder den Bauchumfang noch die jeweiligen Differenzen T1-T0 konnten Korrelationen mit den entsprechenden Homocysteinwerten gefunden werden. Auch für das Alter oder für die Höhe des Kreatinins ergab sich kein Zusammenhang mit dem Homocystein. Da für keine weiteren Laborparameter außer für erhöhtes Kreatinin Zusammenhänge mit Homocysteinwerten aus der Literatur bekannt sind, verzichteten wir auf weitere Korrelationsberechnungen bis auf eine Untersuchung des Verhältnisses zwischen Homocystein und hsCRP. Die Mittelwerte beider Parameter steigen in der Lowcarb-Gruppe zum Zeitpunkt T1 an und machen eine genauere Betrachtung interessant. Die Ergebnisse finden sich im folgenden Abschnitt (s. 3.6.2.1.).

Bei nicht vorhandenen Verzehrdaten zum Zeitpunkt T1 konnte keine Korrelationsanalyse zwischen Homocystein und beispielsweise den Vitaminen erfolgen. Mögliche Verbindungen zwischen dem Ausgangsverzehr der Vitamine und dem Homocysteinwert T0 ergaben sich nicht. Die einzige sehr schwache Abhängigkeit mit $r=0,28(p=0,01)$ fand sich für die Zugehörigkeit zu einer bestimmten Diätgruppe und dem Homocysteinwert T1.

Da insgesamt nur ein kleiner Teil der Probandinnen einen hohen Kaffeekonsum oder eine regelmäßige Vitaminsupplementation betrieb und nur wenige aktive oder ehemalige 
Raucherinnen teilgenommen haben, unterblieb der Versuch einer Korrelation dieser Parameter.

\subsubsection{Hoch-sensitives CRP}

\subsubsection{Hoch-sensitives CRP (hsCRP) und Homocystein (HCY)}

Zwischen der Differenz der Homocysteinwerte T1-T0 und der Differenz der hsCRPWerte im selben Zeitraum wie auch zwischen den einzelnen Werten bei T0 oder T1 besteht keine statistisch signifikante Korrelation. Der Rangkorrelationskoeffizient nach Spearman beträgt für den Zeitpunkt T0 r=0,186 ( $\mathrm{P}=0,091), \mathrm{T} 1 \mathrm{r}=0,024(\mathrm{p}=0,831)$ und die Differenz von T0 zu T1 r=0.198 (p=0,072).

\subsubsection{HsCRP, Körpermesswerte und Laborparameter}

Die möglichen Zusammenhänge zwischen dem Ausgangs-hsCRP-Wert und den gemessenen anderen Risikofaktoren für eine Atherosklerose sind in der folgenden Tabelle zusammengestellt:

\section{Korrelationskoeffizienten von hsCRP}

\begin{tabular}{|l|c|c|}
\hline hsCRP T0 mit & Korrelationskoeffizient $\mathbf{~}$ & Signifikanz p \\
\hline Alter & 0,109 & 0,337 \\
\hline BMI T0 & 0,270 & 0,013 \\
\hline Gewicht T0 & 0,204 & 0,063 \\
\hline Bauchumfang T0 & 0,108 & 0,329 \\
\hline Glucose T0 & 0,218 & 0,049 \\
\hline Triglyceride T0 & 0,145 & 0,189 \\
\hline Gesamtcholesterin T0 & 0,118 & 0,287 \\
\hline LDL-Cholesterin T0 & 0,070 & 0,527 \\
\hline HDL-Cholesterin T0 & 0,056 & 0,610 \\
\hline
\end{tabular}

Tabelle 38: Korrelationskoeffizienten von hsCRP

Die einzigen signifikanten Korrelationen bestehen zwischen BMI und hsCRP und Glucose und hsCRP. Alter, Gewicht, Bauchumfang oder die einzelnen Lipidparameter korrelieren nicht mit dem Entzündungsmarker.

Für die Änderungen der Parameter und die Änderungen des hsCRP zwischen den Zeitpunkten T0 und T2 ergibt sich folgendes Bild: 
Korrelationen der hsCRP-Differenz T2-T0

\begin{tabular}{|l|c|c|}
\hline hsCRP T2-T0 mit & Korrelationskoeffizient $\mathbf{r}$ & Signifikanz $\mathbf{p}$ \\
\hline hsCRP T2T0 & $-0,681$ & $<0,0001$ \\
\hline BMI T2-T0 & 0,163 & 0,139 \\
\hline Gewicht T2-T0 & 0,179 & 0,103 \\
\hline Bauchumfang T2-T0 & 0,092 & 0,405 \\
\hline Glucose T2-T0 & 0,069 & 0,539 \\
\hline Triglyceride T2-T0 & 0,125 & 0,257 \\
\hline Gesamtcholesterin T2-T0 & 0,073 & 0,521 \\
\hline LDL-Cholesterin T2-T0 & 0,076 & 0,492 \\
\hline HDL-Cholesterin T2-T0 & 0,185 & 0,091 \\
\hline
\end{tabular}

Tabelle 39: Korrelationen der hsCRP-Differenz T2-T0

Lediglich der Zusammenhang zwischen Ausgangs-hsCRP und der Änderung dieses Parameters über die Zeit ist hoch signifikant. Ansonsten ergeben sich keine signifikanten Korrelationen, auch nicht zwischen hsCRP-Änderung und BMI-Änderung. Korrelationen zwischen den Nährstoffdaten und dem hsCRP gibt es nicht.

\subsubsection{Körpermesswerte, Labordaten und Kalorienverzehr}

Der BMI korreliert als einziges Körpermaß mit den Blutdruck- und Glucosewerten. Für die Differenzen der systolischen und diastolischen Blutdruckwerte finden sich signifikante Korrelationen mit den BMI-Differenzen T2-T0: $r=0,254(\mathrm{p}=0,021)$ für die systolische und $\mathrm{r}=0,240(\mathrm{p}=0,029)$ für die diastolische Blutdruckänderung. Allerdings sind die Korrelationen sehr schwach ausgeprägt.

Ein signifikanter Zusammenhang konnte ebenfalls zwischen Nüchternglucosewert und BMI identifiziert werden. Das ist zu den Zeitpunkten T0 und T2 für alle Teilnehmerinnen sichtbar. Der Pearson-Korrelationskoeffizient beträgt bei T0 r=0,334 ( $\mathrm{p}=0,002)$ und bei T2 $\mathrm{r}=0,224(\mathrm{p}=0,043)$. Allerdings ergibt sich für die Änderung der Glucose von T0 zu T2 keine signifikante Abhängigkeit von der BMI-Veränderung. 


\section{Abhängigkeit der Nüchternglucose vom BMI}
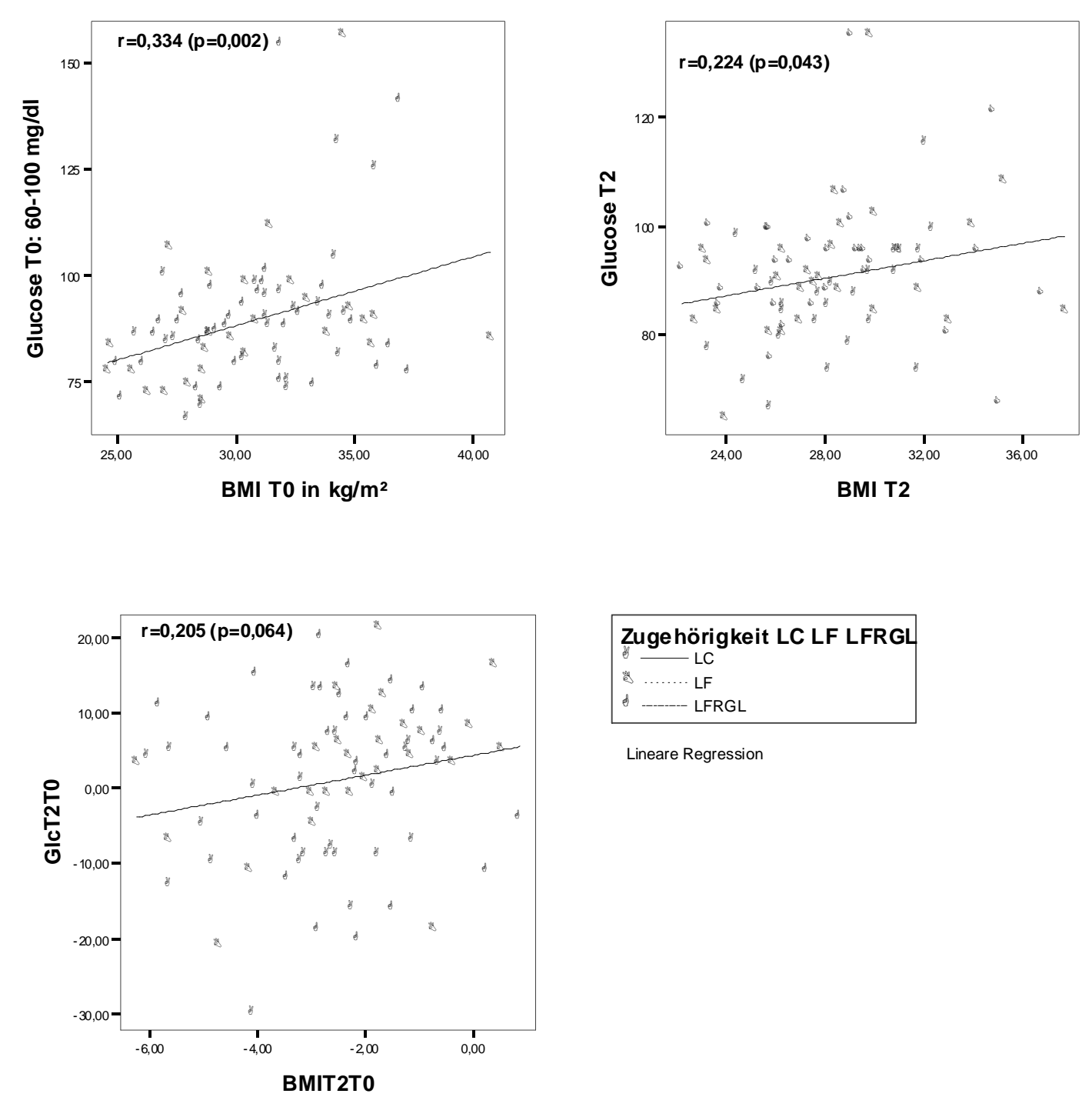

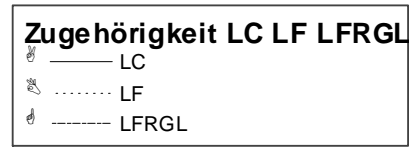

Lineare Regression

Abbildung 6: Korrelation Glucose und BMI

Für die Lipide wurde keine nähere Analyse der Veränderungen in der vorliegenden Untersuchung durchgeführt, da dies Gegenstand einer eigenen Arbeit sein wird. Bei den anderen Blutparametern finden sich keine signifikanten Veränderungen und daher wurden ebenfalls keine weiteren Untersuchungen mit ihnen durchgeführt.

Der Vergleich des Gewichtsverlusts mit der durchschnittlichen Verringerung der Kalorien zwischen den Zeitpunkten T0 und T2 in den einzelnen Gruppen legt einen Zusammenhang zwischen diesen Parametern nahe: Die größte Gewichtsabnahme und die größte Abnahme im Kalorienverzehr finden sich in der Gruppe LC, auch wenn die Unterschiede gering und statistisch nicht signifikant sind. Dagegen erzielte die Gruppe LF durchschnittlich das geringste Gewichts- und Kaloriendefizit. Während in der Gruppe LC die 
Teilnehmerinnen -651,8 kcal täglich weniger verzehrten und 8,1 kg abnnahmen, beträgt das Kaloriendefizit im Mittel in LF nur -394,9 kcal bei -6,3 kg und in LFRGL -592,6 kcal bei -6,8 kg Gewichtsreduktion. Der scheinbare Zusammenhang erweist sich in der Berechnung jedoch als falsch. Eine statistisch signifikante Korrelation läßt sich nicht finden, da die einzelne Probandin mit großem Kaloriendefizit in der Ernährung nicht unbedingt auch einen großen Gewichtsverlust erreichen konnte.

\section{Abhängigkeit der Gewichtsabnahme von der Kalorienaufnahme}
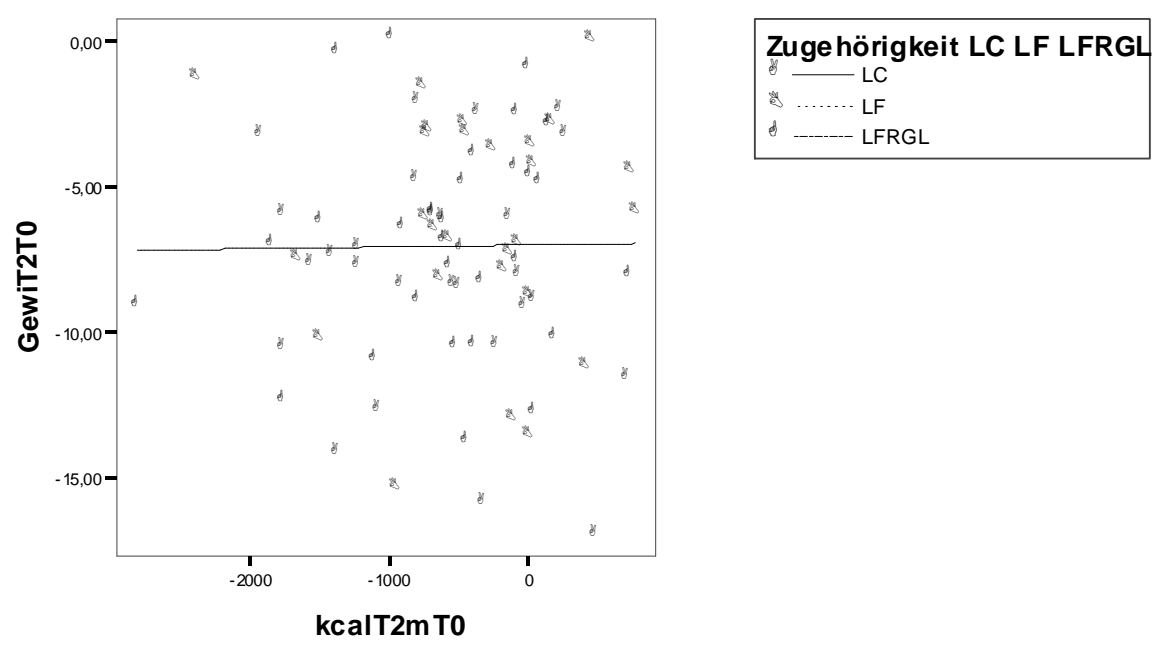

Abbildung 7: Abhängigkeit der Gewichtsabnahme von der Kalorienaufnahme

\subsubsection{Verzehrdaten}

\subsubsection{Kohlenhydrate und Vitamine}

Besonders in der Low-carb-Gruppe mit deutlich verringertem Kohlenhydratverzehr sinkt die Menge der Folsäure- und Vitamin-B6-Aufnahme signifikant. Statistisch ergibt sich eindeutig ein positiv linearer Zusammenhang zwischen dem Kohlenhydratverzehr und der Aufnahme dieser beiden Vitamine. Für die Änderung des Kohlenhydratverzehrs und der Folsäurezufuhr lautet der Korrelationskoeffizient $r=0,474$ ( $p<0,0001)$ und für die Änderung der Kohlenhydrataufnahme und der Vitamin-B6-Zufuhr $r=0,451$ ( $\mathrm{p}<0,0001)$. Die Vitamin-B12Versorgung ist vom Kohlenhydratverzehr unabhängig mit $\mathrm{r}=0,023(\mathrm{p}=0,833)$. 
Abhängigkeit der Vitaminaufnahme vom Kohlenhydratverzehr
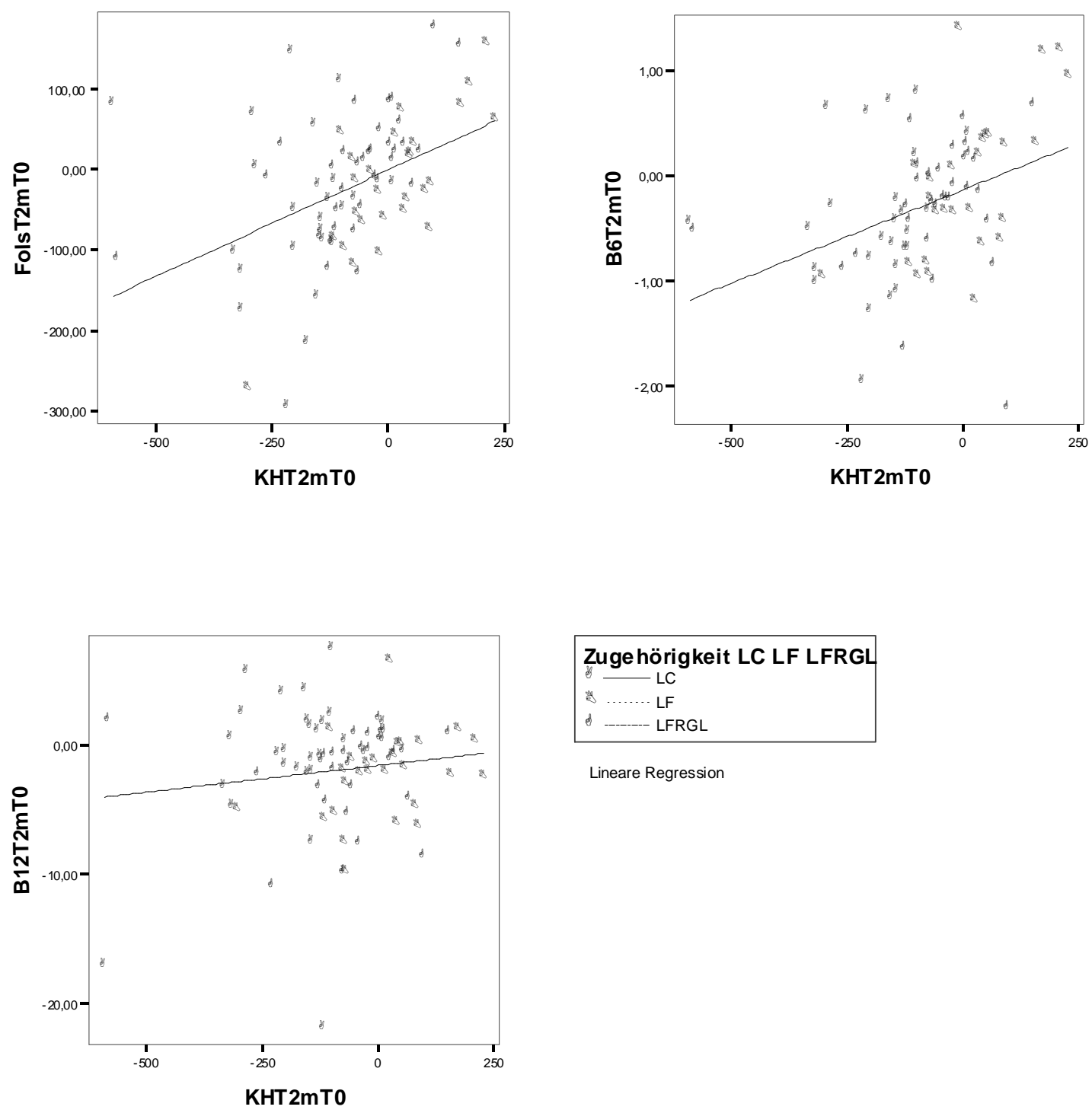

Lineare Regression

Abbildung 8: Abhängigkeit der Vitaminaufnahme vom Kohlenhydratverzehr 


\section{Diskussion}

\subsection{Zusammensetzung der Diätgruppen}

Durch die zufällige Verteilung der Teilnehmerinnen auf die drei Diätgruppen mit Ausgleich nach Alter und BMI sind homogene Untersuchungsgruppen entstanden. Die Vergleichbarkeit der Gruppen bezüglich Alter oder BMI wurde durch das nachträgliche Ausscheiden von unterschiedlich vielen Probandinnen in den einzelnen Gruppen nicht beeinflusst. Dies läßt sich anhand der durchgehend geringen Unterschiede in den Ausgangsparametern sehen. Das Gleiche gilt für den Raucherstatus und einen starken Kaffeekonsum bei insgesamt nur wenigen Teilnehmerinnen, die sich als Raucherinnen oder starke Kaffeekonsumentinnen definiert hatten. Lediglich für die von uns nur semiquantitativ erhobenen Daten einer selbständig durchgeführten Vitaminsupplementation einzelner Probandinnen ergeben sich leichte Unterschiede. Gerade im Hinblick auf die Veränderungen der Homocystein- und hsCRP-Werte unter der diätetischen Intervention sind im Einzelfall Beeinflussungen der Ergebnisse denkbar. Dieser Punkt wird in dem jeweiligen Kapitel weiter erörtert.

\subsection{Abbrecherquote}

Sucht man Unterschiede zwischen den verschiedenen Ernährungsgruppen, so ist die ungleiche Quote an Abbrecherinnen interessant. Insgesamt haben in den 6 Monaten 59 der anfänglich 160 Teilnehmerinnen die Teilnahme an dem Untersuchungsprogramm vorzeitig beendet. Das sind 36,9\% aller Teilnehmerinnen. Für eine Untersuchung mit diätetischer Intervention ist dies eine durchaus zu erwartende Zahl. Ein direkter Vergleich mit der Untersuchung von Dansinger et al. mit ähnlichen diätetischen Interventionen zeigt nach 6 Monaten eine Ausstiegsquote von 38\% (Dansinger et al.2005). Dabei ergeben sich dort wie hier Unterschiede in der Abrecherquote zwischen den einzelnen Diätgruppen. In unserer Untersuchung fand sich in der kohlenhydratarmen Diätgruppe eine Rate von 45,3\% Abbrecherinnen. Diese Rate lag in den fettreduzierten Gruppen LF und LFRGL bei nur 35,2\% und $30,2 \%$.

Eine genaue Untersuchung von Gründen für das vorzeitige Abbrechen des Programms wird im Rahmen der aktuell vorliegenden Arbeit nicht vorgenommen. Es sollen nur beispielhaft einige der Gründe skizziert werden: So wurden Missfallen der Ernährungsvorgaben, Schwierigkeiten beim praktischen Umsetzen der Diät, logistische Probleme bei Probandinnen 
von außerhalb der Stadt mit den wöchentlichen Terminen zur Gewichtskontrolle, Unzufriedenheit mit der Programmstruktur (nur schriftliche Informationen, keine Gruppensitzungen) und Umzug in eine andere Stadt als Gründe genannt. Einige Teilnehmerinnen hatten bereits bei der ersten Information Einwände gegen eine Low-carb-Diät erhoben. Dies wurde von uns nicht als Ausschlusskriterium bei der Randomisierung berücksichtigt. Vermutlich schieden aus diesem Grund schon in den ersten 4 Wochen 10 Teilnehmerinnen in der Low-carbGruppe aus. In den Gruppen LF beziehungsweise LFGRL brachen mit 6 beziehungsweise 8 im gleichen Zeitraum weniger Personen die Diät ab.

Wenn man die Compliance oder das Gefallen beziehungsweise Missfallen einer Ernährungsweise oder diätetischen Therapie als eines der Gütekriterien in die Bewertung mit aufnimmt, so schneidet die Low-carb-Diät unter deutschen Lebensbedingungen in diesem Punkt schlechter ab als die beiden Low-fat-Varianten. Ein Grund dafür könnte die stärkere Einschränkung der Nahrungsmittelauswahl in der kohlenhydratarmen Gruppe sein. Besonders in Deutschland, wo das Angebot an kohlenhydratarm produzierten und gekennzeichneten Lebensmitteln gering ist gegenüber den USA mit einem Markt für kohlenhydratreduzierte Lebensmittel und „Atkins“-Produkte, fällt dieser Punkt vermutlich ins Gewicht. Allerdings musste auch Dansinger in seiner Untersuchung, die in den USA durchgeführt wurde, in der Low-carb-Gruppe wie auch in der extrem fettarmen Ornish-Diät-Gruppe höhere Abbrecherquoten hinnehmen. Er erklärte diese Tatsache mit der zu extremen Umstellung der Ernährungsweise gegenüber einer herkömmlichen Kost (Dansinger et al. 2005).

\subsection{Veränderung von Gewicht, BMI und Bauchumfang}

Eine signfikante Gewichtsreduktion und damit verbundene Abnahme des BMI und des Bauchumfangs konnte in allen Gruppen erreicht werden. Die leichte Tendenz eines größeren Gewichtsverlusts in der Gruppe LC zu beiden Zeitpunkten T1 und T2 gegenüber den Gruppen LFRGL und LF, letztere mit der geringsten Gewichtsabnahme, ist statistisch nicht signifikant ( $\mathrm{p}=0,4868)$. Diese Ergebnisse decken sich mit den Daten aus der Studie von Dansinger, der insgesamt 4 verschiedene Diätinterventionen miteinander verglich. Er konnte ebenfalls keine signifikanten Unterschiede bezüglich des Gewichtsverlaufs zwischen den Diätgruppen beobachten (Dansinger et al. 2005). Damit widerspricht sein Vergleich einer Low-carb-Diät mit anderen Diäten wie auch die vorliegende Untersuchung den jeweiligen Beobachtungen von Foster und Samaha, die nach 3 bzw. 6 Monaten Ernährungsintervention jeweils signifikant größere Gewichtsverluste unter einer Low-carb-Diät gegenüber Low-fat-Diäten 
gefunden hatten (Foster et al. 2003, Samaha et al. 2003). In beiden Untersuchungen wird allerdings das durch die Diätinterventionen erreichte Kaloriendefizit als Erklärung für die Gewichtsdifferenz herangezogen und nicht diätimmanente Faktoren. Und da in unserer Untersuchung keine signifikanten Unterschiede im Kaloriendefizit nachzuweisen sind, wäre das eine Erklärungsmöglichkeit für die fehlende Signifikanz der Gewichtsdifferenzen zwischen den Gruppen. Andererseits wurde auf den fehlenden Zusammenhang zwischen der Gewichtsabnahme und dem durch die Ernährungsanalyse berechneten Kaloriendefizit bei den einzelnen Teilnehmerinnen hingewiesen. Möglicherweise spielen hier Faktoren wie der Kalorienverbrauch durch Sport und Bewegung eine Rolle. Andere Erklärungsmöglichkeiten ergeben sich durch eventuelle Fehler in den Ernährungsangaben durch die Teilnehmerinnen oder doch durch diätbedingte Faktoren. Diese Punkte können anhand der bisher bekannten Daten und der Ergebnisse dieser Studie nicht geklärt werden.

Verglichen mit anderen Studien ist der Gewichtsverlust in unserer Studie jedoch insgesamt größer. Das zeigt sich nicht nur in der Per-protocol-Analyse. Die Intention-to-treatAuswertung der Gewichtsdaten, in der fehlende Werte nach der Methode Last-oberservationcarried-forward ersetzt wurden, verzeichnet ebenfalls höhere Gewichtsverluste für alle Gruppen. Allerdings bietet nur die Untersuchung von Dansinger ebenfalls eine Per-protocolAnalyse an. Zwischen den beiden Analyseformen macht sich in der vorliegenden Untersuchung die größere Zahl an Abbrecherinnen in der Gruppe LC an einer größeren Differenz der Ergebnisse bemerkbar. Die höhere Anzahl kleiner Gewichtsdifferenzen bei Teilnehmerinnen, die früh die Diät beendeten, führt gegenüber der Per-protocol-Analyse zu einer geringeren mittleren Gewichtsabnahme. Der Unterschied zwischen den beiden Auswertungsformen beträgt in der Gruppe LC 1,8 kg, während er in Gruppen LF und LFRGL bei nur 1,2 und $0,8 \mathrm{~kg}$ liegt.

\subsection{Auswirkungen auf den systolischen und diastolischen Blutdruck}

Dass eine Gewichtsreduktion mit einer Abnahme des systolischen und diastolischen Blutdrucks einhergeht, ist bekannt. In dieser Untersuchung verringerten sich die systolischen und diastolischen Blutdruckwerte im Zuge der Gewichtsabnahme. Insgesamt kommt es in allen 3 Gruppen zu einer signifikanten systolischen Blutdrucksenkung im Verlauf des 6monatigen Beobachtungszeitraumes. Das trifft für die Gruppen LC und LF auch für den diastolischen Blutdruck zu, nicht aber in der Gruppe LFRGL. Hier fällt die diastolische 
Blutdrucksenkung nicht signifikant aus. Eine Erklärung hierfür ist am ehesten über die unterschiedliche antihypertensive Medikation bei einzelnen Probandinnen möglich. Durch die Einnahme von zum Teil mehreren antihypertensiven Medikamenten pro Teilnehmerin und der Anpassung der Therapie im Verlauf der Gewichtsreduktion ist eine genaue quantitative Analyse der Auswirkungen der Diäten auf den Blutdruck in dieser Erhebung schwer möglich. Sicher lässt sich jedoch sagen, dass sich die Befürchtung einer Blutdrucksteigerung durch die fett- und vermutlich natriumreichere Ernährung in der Low-carb-Gruppe nicht bestätigt hat.

\subsection{Auswirkungen auf den Homocysteinspiegel}

Eines der interessantesten Ergebnisse dieser Untersuchung ist der Homocysteinanstieg, den wir nach den ersten 4 Wochen mit den restriktivsten diätetischen Vorgaben messen konnten. Der Anstieg fällt nur in der kohlenhydratarmen Diät-Gruppe statistisch signifikant aus. Die mittleren Ausgangswerte lagen bei allen Gruppen im Normbereich mit einer geringen Streuungsbreite. Da unter stabilen Lebensbedingungen Homocystein über einen Zeitraum von Wochen bis zu mehreren Jahren ein wenig variabler Parameter ist (Nurk et al. 2004), muss dieser deutliche Anstieg auf die untersuchungsinduzierten Veränderungen zurückzuführen sein. Bemerkenswert ist die Verdopplung bis Verdreifachung des Homocysteinspiegels bei einzelnen Probandinnen, wohingegen bei anderen nur ein leichter Anstieg oder gar ein leichter Abfall der Werte sichtbar wird.

Einige mögliche Auslöser von Homocysteinveränderungen wie Änderungen im Rauchverhalten, im Kaffeekonsum oder in der Vitaminsubstitution spielen in unserem Teilnehmerinnenkollektiv keine Rolle. Aus der Hordaland Homocysteine Study geht hervor, dass es einen inversen Zusammenhang zwischen Gewichtsentwicklung und Homocysteinspiegel gibt. Die wenigen Studien direkt zur Gewichtsreduktion mit Untersuchung des Homocysteinspiegels belegen einen leichten Homocysteinanstieg unter Gewichtsabnahme. Allerdings wird dort ein Anstieg des Homocysteins von 1-2 $\mu$ mol/l in einer Nachuntersuchung 6 bzw. 12 Monaten nach gastrochirurgischem Eingriffzur Gewichtsreduktion (Magenband, Magenbypass) mit sehr hoher durchschnittlicher Gewichtsdifferenz beschrieben (Dixon et al. 2001; Sheu et al. 2001). Aus unseren Daten läßt sich eine signifikante Korrelation zwischen Gewichtsabnahme und Homocysteinanstieg nicht ableiten. Ein derartiger Zusammenhang wäre angesichts des spontanen Abfalls des Homocysteins im Verlauf der Studie unter weiter zunehmender Gewichtsabnahme auch unwahrscheinlich gewesen. 


\section{Abhängigkeit der Homocysteinänderung von der Gewichtsreduktion}
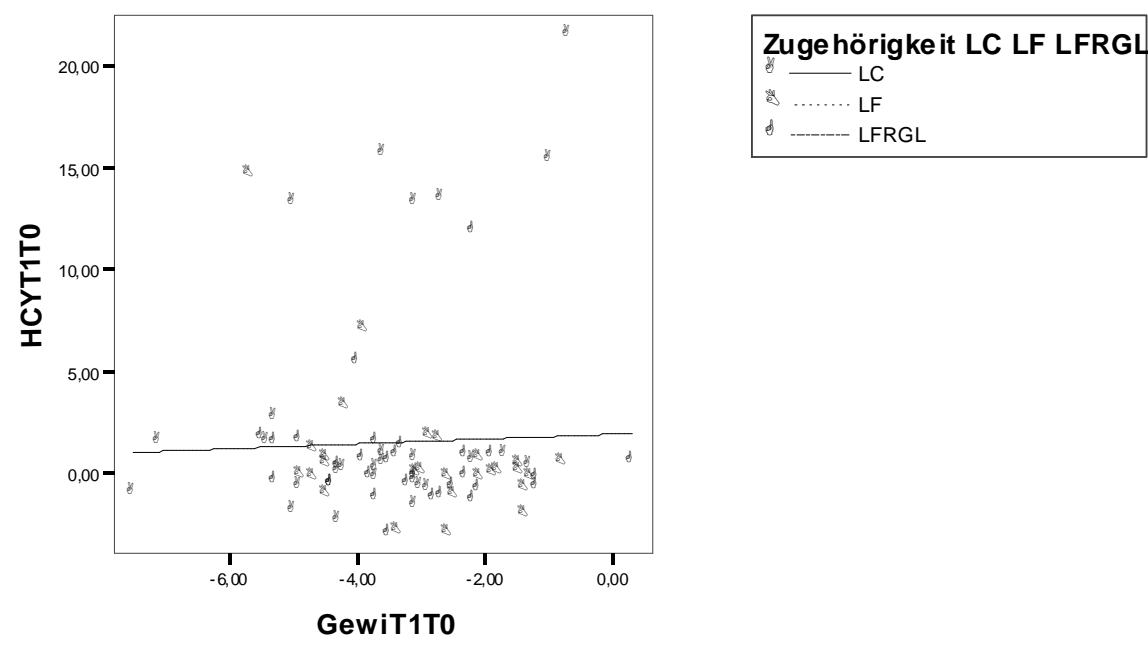

Abbildung 9: Gewichtsreduktion und Homocysteinveränderung

Andere Einflussfaktoren auf das Homocystein im Serum, die einen kurzfristigen und hohen Anstieg erklären könnten, sind eine akute Methioninbelastung des Körpers wie sie beispielsweise im Rahmen eines Methioninbelastungstests stattfindet oder ein akuter Mangel an den Vitaminen Folsäure, Vitamin B12 und/ oder B6. Ein Mehrverzehr von Protein und damit von Methionin kann anhand der vorhandenen Daten nicht belegt werden. Weil nicht nach 4 Wochen sondern erst wieder nach 6 Monaten der Diät eine Ernährungserhebung durchgeführt wurde, können genaue Aussagen zum Protein- oder Vitaminverzehr zum Zeitpunkt des Homocysteinanstiegs nicht gemacht werden. Nach 6 Monaten zeigt sich allerdings ein Minderverzehr von Eiweiß, auch in der Low-carb-Gruppe, was gegen eine mögliche Vermehrung der Methioninbelastung spricht. Auch ergeben sich aus den Daten der Hordaland Homocysteine Study Hinweise, dass auf längere Sicht eine proteinreichere Kost mit niedrigeren Homocysteinwerten einhergeht. Die erhöhten Homocysteinwerte nach einem Methioninbelastungstest werden dagegen nach 6 Stunden gemessen. Aus diesem Grund kann über die Auswirkungen einer 4-wöchigen gesteigerten Proteineinnahme auf das Homocystein nur spekuliert werden. Derartige Überlegungen finden sich beispielsweise in einem Editorial des American Journal of Clinical Nutrition von Verhoef und Katan (Verhoef und Katan 2004). Genaue Daten dazu lassen sich in der Literatur nicht finden.

Dagegen gibt es konkrete Daten zu einem Vitaminmangel und der Erhöhung des Homocysteinspiegels, wobei der Zusammenhang eines Folsäuremangels mit einer Hyperhomocysteinämie am eindeutigsten diskutiert wird. Aus den Werten des Kohlenhydrat- und 
Folsäureverzehrs nach 6 Monaten, den bereits niedrigen Ausgangswerten des Folsäureverzehrs und der noch stärkeren Beschränkung der Kohlenhydratzufuhr in den ersten 4 Wochen der Diät in der Low-carb-Gruppe lässt sich für sie eine vitaminärmere Ernährung zum Zeitpunkt T1 sicher annehmen. Der Zusammenhang zwischen dem Verzehr von Folsäure und Vitamin B6 und der Kohlenhydrataufnahme ist eindeutig linear. Die wahrscheinlichste Ursache des Homocysteinanstiegs ist daher ein Folsäuremangel.

Nach Refsum et al. steigt Homocystein bereits bei niedrig-normalen Folsäurewerten an und kehrt bereits 1 bis 2 Wochen nach ausreichender Folsäurezufuhr wieder auf Normalwerte zurück (Refsum et al. 2004). Damit kann der kurzfristige Anstieg unter strengster Low-carbDiät und die anschließende Rückkehr zum Ausgangsniveau zum Zeitpunkt T2 sinnvoll interpretiert werden. Weitere Hinweise für einen Folsäuremangel als Ursache des Homocysteinanstiegs finden sich in einer Studie von Dixon et al.: Sie fanden heraus, dass ein Homocysteinanstieg unter einer Reduktionsdiät nur durch vermehrte Zufuhr von Folsäure und Vitamin B12 verhindert werden kann (Dixon et al. 2001). Anhand dieser Befunde muss ein verminderter Folsäure- und Vitamin B12-Verzehr unweigerlich zum Anstieg des Homocysteinspiegels führen. Da sich in unserer Untersuchung der Vitamin-B12-Verzehr in der LC-Gruppe durch den verstärkten Fett- und Fleischverzehr nicht signifikant verringert, ergibt sich kein Hinweis für einen Vitamin-B12-Mangel bei unseren Probandinnen. Die Vitamin-B12-Aufnahme ist in allen 3 Gruppen und zu beiden Zeitpunkten der Ernährungserhebung im Normbereich des täglichen Bedarfs.

Für einen mangelhaften Verzehr von Vitamin B6 und eine Hyperhomocysteinämie gibt es ebenfalls Hinweise aus der Literatur. Die Vitamin-B6-Aufnahme ist zu T0 und T2 bedarfsdeckend. Wegen des positiv linearen Zusammenhangs der Vitamin-B6- und KohlenhydratAufnahme und der deutlich verringerten Vitamin-B6-Zufuhr in der Gruppe LC zum Zeitpunkt T2 wäre eine defizitäre Versorgung mit diesem Vitamin zum Zeitpunkt T1 durchaus möglich. Ein Vitamin-B6-Mangel wäre demnach als Ursache des Homocysteinanstiegs gleichfalls denkbar oder eine Kombination aus Folsäure- und Vitamin-B6-Mangel.

Die Tatsache, dass keine der Teilnehmerinnen mit Angabe einer Vitaminsupplementation einen massiven Homocysteinanstieg zum Zeitpunkt T1 hatte, bestärkt die Vitaminmangel-Hypothese. Der maximale Anstieg von Homocystein unter gelegentlicher oder regelmäßiger Vitamineinnahme lag bei 5,3 $\mu$ mol/l. Hier könnte die unterschiedliche Zahl an Probandinnen mit Vitamineinnahme Einfluss auf die Gruppenergebnisse für Homocystein nehmen: Nur 2 Probandinnen in der Gruppe LC nahmen vor und in den ersten 4 Wochen der 
Diätintervention Vitamine ein gegenüber 4 und 9 Teilnehmerinnen in den beiden anderen Gruppen. Diese Zahlen lassen die Annahme zu, dass gerade in der Low-carb-Gruppe mit diätbedingt wahrscheinlichem Folsäure- und Vitamin-B6-Mangel keine Überdeckung des Minderverzehrs durch Vitaminsubstitution auftrat. Dadurch wurde ein signifikanter Homocysteinanstieg in dieser Gruppe eventuell erst ermöglicht.

Bei durchschnittlichem Verzehr einer gesunden Mischkost und bei normalem Vitaminbedarf sollte in Deutschland eine ausreichende Versorgung mit allen Vitaminen normalerweise gesichert sein. Die Lebensmittellagerung und Nahrungszubereitung mit Erhitzen führt zu einem hohen Verlust von intakter Folsäure in der Nahrung. Daher gilt dieses Vitamin auch in Deutschland generell als kritisches Vitamin bezüglich der ausreichenden Versorgung (DGE et al. 2000). Bei bedarfsdeckender Aufnahme bleibt eine zusätzliche Vitaminsubstitution ohne Effekt auf den Homocysteinwert. Die vergleichbaren Homocysteinmittelwerte zum Zeitpunkt T0 in allen 3 Gruppen sind daher trotz unterschiedlicher Anzahl von Teilnehmerinnen mit Vitamineinnahme erklärbar. Im Rahmen einer Reduktionskost ändert sich die Situation jedoch. Wegen der Gefahr der Minderversorgung vor allem bei erhöhtem Bedarf in Schwangerschaft und Stillzeit, aber auch im Alter und bei Alkoholabusus, werden in den USA, Canada und anderen Ländern verschiedene Lebensmittel bereits mit Folsäure angereichert. Auch wird in Studien mit diätetischer Intervention zur Vermeidung eines Vitaminmangels dort häufiger eine Vitmaminsubstitution durchgeführt, wie zum Beispiel in der bereits genannten Untersuchung von Dansinger. Das Ausbleiben von negativen Effekten einer Low-carb-Kost in diesen Ländern und unter anderen Studienbedingungen kann somit erklärt werden.

\subsubsection{Hyperhomocysteinämie und MTHFR-Genotyp}

Um weitere Möglichkeiten für die Homocysteinänderungen zu untersuchen, wurde der MTHFR-Genotyp bei allen Teilnehmerinnen mit Homocysteinanstieg sowie in der gesamten Gruppe LC als Kontrollkollektiv bestimmt. Es zeigte sich kein Zusammenhang zwischen einem bestimmten Genotyp und Veränderungen des Homocysteins. Die einzigen beiden Teilnehmerinnen mit homozygoter Mutation des MTHFR-Gens, also mit eingeschränkter Enzymaktivität der MTHFR, zeigten keinen Anstieg des Homocysteins. Der überwiegende Teil der Probandinnen mit einem signifikanten Anstieg des Homocystein kam dagegen aus dem Normalkollektiv ohne eine Mutation des für MTHFR codierenden Gens; das gilt für die Lowcarb-Gruppe und die 3 Teilnehmerinnen aus den beiden fettarmen und kohlenhydratreichen Gruppen. Von den 8 Frauen mit einer heterozygoten Mutation hatten nur 2 eine Erhöhung des 
Homocysteinspiegels. Diese Ergebnisse lassen keinen Zusammenhang zwischen dem Genotyp und einem Homocysteinanstieg erkennen.

Allerdings haben die homo- und heterozygoten Trägerinnen der MTHFR-Mutation insgesamt einen etwas höheren durchschnittlichen Homocysteinausgangswert gegenüber den Wildtyp-Trägerinnen. Diese Ergebnisse decken sich mit der Literatur und sind möglicherweise auf einen erhöhten Bedarf an Vitaminen bei verminderter MTHFR-Aktivität zurückzuführen. Gleichzeitig müsste aus diesen Tatsachen abgeleitet werden können, dass besonders die Probandinnen mit einer Genmutation keinen klinisch wirksamen Vitaminmangel besaßen.

\section{Homocysteinwerte in Abhängigkeit vom Genotyp des für MTHFR codierenden Gens}

\begin{tabular}{|c|c|c|}
\hline Genotyp & CC & CT und TT \\
\hline $\mathrm{N}$ & 19 & 10 \\
\hline & \multicolumn{2}{|c|}{ Homocystein in $\boldsymbol{\mu m o l} / \mathbf{I}$} \\
\hline Mittel & 9,4 & 9,8 \\
\hline SD & 2,2 & 2,1 \\
\hline Min. & 5,3 & 7,2 \\
\hline Max. & 12,8 & 14 \\
\hline
\end{tabular}

Tabelle 40:Homocysteinausgangswerte T0 in Abhängigkeit vom Genotyp

\subsection{Hoch-sensitives CRP}

Anders als es aus anderen Studien zur Gewichtsreduktion bekannt ist, sinkt der hsCRPWert in dieser Studie nicht in allen 3 Untersuchungsgruppen mit der Gewichtsabnahme. In der kohlenhydratarmen Gruppe ist nach den ersten 4 Wochen (T1) unter der Diät sogar ein signifikanter Anstieg des hsCRP zu erkennen. Zum Zeitpunkt T2 nach 6 Monaten sind die Werte wieder gesunken. Insgesamt kommt es dadurch über den 6-monatigen diätetischen Interventionszeitraum nicht zu einer Reduktion des hsCRP, obwohl in der Low-carb-Gruppe mit $-8,1 \mathrm{~kg}$ sogar die größte Gewichtsabnahme erreicht wurde. In den beiden fettarmen Diätgruppen kann dagegen eine stetige und statistisch signifikante Verminderung dieses Entzündungsparameters über die beiden Zeitpunkte T1 und T2 nachgewiesen werden.

Obwohl ein Zusammenhang zwischen BMI und hsCRP-Werten gefunden wurde, fehlt dieser zwischen den Veränderungen dieser Parameter. Diese Ergebnisse decken sich nicht mit den bisherigen Literaturdaten, die auch eine gleichsinnige Korrelation zwischen Gewichtsabnahme und hsCRP-Verminderung nachweisen konnten (Dansinger et al. 2005; Esposito et al. 2003; Esposito et al. 2004). Unter diätetischer Therapie mit Gewichtsreduktion belegen die 
genannten Studien eine Verbesserung des Entzündungsmarkers hsCRP. Bei Dansinger et al. war diese Effekt auch unabhängig von der Art der Diät: Die Autoren konnten eine ähnlich große hsCRP-Verbesserung unter der Atkins-Diät wie unter der sehr fettarmen Ornish-Diät beobachten. Das erscheint erstaunlich, da die Nahrungszusammensetzung dieser beiden Diätvarianten direkt gegensätzlich ist. Es lässt auf einen fehlenden Zusammenhang zwischen Fettund Kohlenhydratgehalt der Nahrung und dem Entzündungsparameter hsCRP schließen.

Für unsere Untersuchung wirft das die Frage nach der Ursache des initialen Anstiegs und des insgesamt fehlenden Rückgangs des hsCRP in der Low-carb-Gruppe auf. Von den Ausgangswerten her unterscheiden sich die 3 Gruppen nicht. Lediglich die Ernährungsweise weicht laut Studienprotokoll erheblich voneinander ab. Eine direkt oder indirekt diätetisch bedingte hsCRP-Zunahme in der Low-carb-Gruppe liegt daher nahe. Mögliche Einflussfaktoren auf den hsCRP-Spiegel wie Blutdruck, Lipide und Glucose haben wir gemessen und konnten keine direkten Zusammenhänge zum hsCRP finden. Akute Entzündungsreaktionen des Körpers, beispielsweise durch Infekte, hoffen wir durch den Cut-off bei $8 \mathrm{mg} / \mathrm{l}$ als Einflussfaktoren ausschließen zu können. Die zum Zeitpunkt T0 und T1 ebenfalls gemessenen Homocysteinwerte zeigen trotz des Anstiegs beider Parameter keine direkte Korrelation zueinander. Da der deutliche hsCRP-Anstieg jedoch nur in der Low-carb-Gruppe und zum gleichen Zeitpunkt wie der Homocysteinanstieg zu finden war, bleibt eine Verbindung dieser beiden Ergebnisse dennoch wahrscheinlich.

Ein möglicher Zusammenhang ergibt sich aus der gesicherten Abhängigkeit beider Parameter von verschiedenen Vitaminen: Homocystein ist bekanntlich abhängig von Folsäure, Vitamin B6 und B12. Folsäure hat einen protektiven Effekt auf die Gefäßinnenhaut und beugt auch unabhängig von einer Hyperhomocysteinämie einer endothelialen Dysfunktion und dem Auftreten von Herz-Kreislauf-Erkrankungen vor. Ein Vitamin-B6-Mangel bewirkt eine Beeinträchtigung des Immunsystems und des Proteinmetabolismus. Friso et al. konnten eine Assoziation des hsCRP-Spiegels mit der Höhe des Vitamin-B6-Wertes im Blut finden (Friso et al. 2001). Gleichzeitig konnte auch gezeigt werden, dass bei erhöhtem Bedarf die orale Aufnahme nicht mit den Blutspiegeln von Vitamin B6 korreliert. Nimmt man nun einen erhöhten Bedarf für Vitamin B6 und für Folsäure im Rahmen eines Homocysteinanstiegs mit negativen Auswirkungen auf den menschlichen Körper an, sind besonders bei mangelhafter Zufuhr dieser Vitamine entzündliche Vorgänge mit Anstieg des hsCRP vorstellbar.

Darüber hinaus sind entzündliche Phänomene für Mangelzustände anderer Vitamine, vor allem der so genannten Oxidanzien wie Vitamin C und E, ebenfalls denkbar: Die plötzli- 
che Minderung der oxidativen Kapazität des Körpers durch mangelhafte orale Zufuhr dieser Substanzen kann möglicherweise zu einem Anstieg des oxidativen Stresses und einer Entzündungsantwort führen. Letztere könnte sich in einer vermehrten hsCRP-Präsenz im Serum niederschlagen. Ein inverser Zusammenhang zwischen der Höhe der Vitamin-C-Aufnahme und kardiovaskulären Erkrankungen konnte nachgewiesen werden (Hahn 2003).

Eine kanadische Untersuchung zu kohlenhydratarmer Diät mit Erfassung der Nährstoffzusammensetzung mittels Ernährungstagebuch fand innerhalb eines 10-wöchigen Zeitraums unter anderem eine Halbierung des Verzehrs von Vitamin $\mathrm{C}$ und eine deutliche Verminderung von Vitamin B1, B2, B6, D, A und Folsäure (Meckling et al. 2004). HsCRP wurde nicht mit gemessen. Die mangelhafte Vitaminversorgung unter kohlenhydratarmer Kost ohne Vitaminsupplementation erscheint eindeutig, ein Zusammenhang mit dem hsCRP-Anstieg in unserer Studie bleibt spekulativ. Verschiedene Mechanismen und der Mangel verschiedener Vitamine können als Ursache vermutet, aber hier nicht bewiesen werden. Die Klärung dieser Hypothesen erfordert weitere Untersuchungen.

Dass in der Vergleichsstudie von Dansinger et al. kein negativer Effekt auf das hsCRP unter der streng kohlenhydratarmen Ernährung ( $<20 \mathrm{~g} /$ Tag bis maximal $50 \mathrm{~g} / \mathrm{Tag}$ ) zu erkennen war, hängt möglicherweise mit der empfohlenen Vitaminsupplementation zusammen. Allen Studienteilnehmern wurde laut Protokoll zu Beginn der Studie die tägliche Einnahme einer nicht verschreibungspflichtigen Multivitamintablette unabhängig von der Diätgruppe angeraten. Auf diese Maßnahme hatten wir bewusst verzichtet, um realitätsnahe Bedingungen zu schaffen. Eine Vitaminsupplementation ist in Deutschland nicht unbedingt üblich. Wir erhielten so die Möglichkeit, gegebenenfalls negative Folgen verschiedener diätetischer Ernährungsinterventionen aufzudecken, die oft im häuslichen Rahmen ohne medizinische Betreuung und ohne Vitaminsubstitution durchgeführt werden.

\subsection{Glucose und Blutfette}

Im Verlauf von 6 Monaten, dem Zeitraum von T0 zu T2, kam es in keiner der 3 Diätgruppen zu einer signifikanten Abnahme der Blutglucose, wobei alle Glucosemittelwerte im Normbereich liegen. Das Ergebnis ist möglicherweise auf das relativ gesunde Kollektiv von Frauen mit nur sehr wenigen diabetischen Patientinnen zurückzuführen. Die Ausgangswerte der Glucosemessungen ergaben entsprechend insgesamt im Mittel niedrigere Werte als in vergleichbaren Untersuchungen. In einem Untersuchungskollektiv mit mehr diabetischen Menschen wäre ein signifikanter Abfall der Glucose durch eine entsprechende Gewichts- 
reduktion zu erwarten gewesen. Festzustellen bleibt jedoch, dass eine streng kohlenhydratarme Diät (LC: <30 g/Tag) und eine streng fettarme Diät (LF: $<30 \mathrm{~g} / \mathrm{Tag}$ ), wie sie in den ersten 4 Wochen (T0 bis T1) praktiziert wurde, mit einer durchschnittlichen Gewichtsreduktion von ca. $1 \mathrm{~kg} /$ Woche zu einer signifikanten Verringerung der Glucose führt (LC: $\mathrm{p}<0,0001$ und LF: $\mathrm{p}=0,0106)$.

Betrachtet man die Triglyceride, so entsprechen die Ergebnisse weitgehend den Erwartungen aus anderen Studien (Dansinger et al. 2005; Samaha et al. 2003; Stern et al. 2004). Unter einer kohlenhydratarmen Diät fallen die Triglyceride signifikant ab (LC: $\mathrm{p}=0,0022$ ) während dies bei den fettarmen Diätvarianten (LF: $\mathrm{p}=0,4670$ und LFRGL: $\mathrm{p}=0,9617$ ) oder konventionellen Diäten in anderen Studien nicht der Fall ist, unabhängig von der Gewichtsreduktion. Bezüglich der Auswirkungen der kohlenhydratarmen Ernährung auf Gesamtcholesterin und LDL-Cholesterin können wir feststellen, dass alle 3 Gruppen signifikante Verringerungen von Gesamt- und LDL-Cholesterin von T0 zu T2 aufweisen. Der leichte Anstieg des HDL-Cholesterins in der Gruppe LC in dieser Untersuchung deckt sich ebenfalls mit den anderen Studienergebnissen, dagegen bleibt eine signifikante Verminderung des HDL unter fettarmer Ernährung, wie sie in einigen Untersuchungen gefunden wurde, bei uns aus.

\subsection{Harnsäure, Nieren- und Leberwerte}

Die Leber- und Nierenwerte sowie die Harnsäure zeigen bis auf eine leichte Reduktion der Alanin-Glutamyl-Transferase (ALT/GPT) in allen Gruppen und eine geringe Verminderung der Alkalischen Phosphatase (AP) besonders in der Low-carb-Gruppe keine nennenswerten Veränderungen. Ein Anstieg dieser Werte war in keiner der Gruppen zu verzeichnen. Damit konnten wir nachweisen, dass ein theoretisch denkbarer Anstieg der Harnsäure unter kohlenhydratreduzierter und fleisch- und vermutlich purinreicher Kost bei gleichzeitiger Gewichtsreduktion nicht eintritt. Dasselbe gilt für Kreatinin und Harnstoff, zwei Markern der Nierenfunktion, die unter Low-carb-Diäten bei möglichem Mehrverzehr von Eiweiß eventuell ansteigen könnten. Da wir keinen erhöhten Eiweißverzehr finden konnten, wäre ein Anstieg dieser Parameter allerdings verwunderlich gewesen. Sicherheitsbedenken gegen kohlenhydratarme, fleischreiche Diäten lassen sich aus diesen Ergebnissen nicht ableiten. 


\subsection{Verzehrdaten von Kalorien, Makronährstoffen und ausgewählten Mikronährstoffen}

Die Daten der Ernährungserfassung geben uns die Möglichkeit der Betrachtung von Zusammenhängen zwischen Körper- und Laborparametern und einzelnen Nährstoffen und zusätzlich die Chance, die Compliance der Probandinnen abzuschätzen. Recht deutliche Veränderungen im Essverhalten sind in allen 3 Untersuchungsgruppen zu erkennen, wenn auch im Mittel nur die Gruppe LF mit einer Einschränkung des Fettverzehrs auf täglich maximal $45 \mathrm{~g}$ ihre Zielvorgabe überwiegend einhielt. Die Gruppe LC nahm ca. $40 \%$ mehr an Kohlenhydraten zu sich als die vorgegebenen 60 g. Für die Gruppe LFRGL ist ebenso ein etwas über den Vorgaben liegender Fettverzehr und gleichzeitig ein nur geringer Mehrverzehr von Ballaststoffen nachweisbar. Letzteres läßt die konsequente Beachtung der Beschränkung der Kohlenhydrate mit hoher glykämischer Last zumindest fraglich erscheinen.

Die Einhaltung von Zufuhrempfehlungen für bestimmte Nahrungsinhaltsstoffe ist für die gesundheitliche Unbedenklichkeit einer Ernährung wichtig. Durch die Verzehrdiagnostik wurde die ausreichende Versorgung mit wichtigen Nährstoffen unter den diätetischen Vorgaben und hiesigen Lebensbedingungen erst ermöglicht. Ein erstes Ergebnis dieser Analyse ist der Minderverzehr von Ballaststoffen in der kohlenhydratarmen Diätgruppe. Mit einem Verzehr von durchschnittlich nur noch $18 \mathrm{~g}$ Ballaststoffen pro Tag wurde die empfohlene Menge von 30 g/Tag deutlich unterschritten. Die kohlenhydratarme Ernährungsempfehlung ist damit als gesundheitlich bedenklich einzustufen. Eine langfristige ballaststoffarme Ernährung, wie in der Gruppe LC, kann aus ernährungsmedizinischer Sicht auch deshalb nicht empfohlen werden.

Die Daten der einzelnen Fettsäuren und der B-Vitamine erlauben eine differenzierte Betrachtung ihrer realen Verzehrmenge im Kontext der jeweiligen diätetischen Vorgaben unter deutschen Ernährungsbedingungen. Während der Anteil an gesättigten Fettsäuren in der Nahrung der Teilnehmerinnen in den fettarmen Untersuchungsgruppen stärker zurückgeht als der an einfach und mehrfach ungesättigten Fettsäuren, ist der Mehrverzehr von Fett in der Gruppe LC nur mit einem unwesentlichen Anstieg von gesättigten Fettsäuren verbunden. Dies ist aus gesundheitlicher Sicht sicher positiv zu bewerten. Angesichts der Philosophie und der Diätvorschlage von Dr. Atkins überrascht dieses Ergebnis (Atkins und Herwood 1974).

Negativ sieht besonders der Vergleich der Verzehrmenge mit der Zufuhrempfehlung der DGE für Folsäure aus: Bei insgesamt niedriger Zufuhr von Folsäure bereits vor den 
Diätinterventionen ist die weitere Abnahme derselben unter diätetischer Einschränkung in der Low-carb-Gruppe signifikant $(\mathrm{p}=0,0038)$ und kann gesundheitliche Nebenwirkungen haben. In den beiden Low-fat-Gruppen gibt es keine bedeutsame Änderung der Folsäureaufnahme (LF: p=0,2419 uns LFRGL: p=0,6170). Zudem kommt es, ebenfalls in der kohlenhydratarmen Diätgruppe, zu einem signifikanten Minderverzehr von Vitamin B6 (p=0,0029). Letzterer ist aber auch in der Gruppe LFRGL zu finden (p=0,0230). In dieser Gruppe kann der eingeschränkte Genuss einzelner Obstsorten mit hoher glykämischer Last als Ursache des Minderverzehrs vermutet werden. Vitamin B12 wird in den fettarmen Diätgruppen in signifikant reduziertem Maß aufgenommen. Die Zufuhrempfehlungen von Vitamin B6 und B12 wurden jedoch zu den Zeitpunkten T0 und T2 in allen 3 Gruppen eingehalten. Diese Ergebnisse lassen eine Vitaminsupplementation zumindest mit Folsäure und unter einer Lowcarb-Diät sinnvoll erscheinen, um gesundheitliche Nachteile zu vermeiden. Da für eine niedrig dosierte Vitaminsupplementation in empfohlener Tagesdosierung keine negativen Nebenwirkungen bekannt sind, sollte zum Schutz vor einem Vitaminmangel unter Reduktionsdiäten generell die Einnahme entsprechender Multivitaminpräparate diskutiert werden.

Die Auswertung von Ernährungserhebungen muss jedoch insgesamt mit Vorsicht interpretiert werden. Im Hinblick auf Ernährungsbefragungen bei diätetischen Maßnahmen sind systematische Fehler aufgrund von psychologischen Faktoren prinzipiell möglich. Das Verschweigen von Snacks und die vermehrte Angabe von erwünschten Nahrungsmitteln können, wie auch das Vergessen von Mahlzeiten in der retrospektiven Abfrage des Verzehrs, zu Ungenauigkeiten und Unstimmigkeiten der Daten führen (Jarzemski 2005; Faustin 2004). 


\section{Zusammenfassung}

In dieser randomisierten offenen klinischen Vergleichstudie beobachteten wir 160 Probandinnen unter 3 aktuell diskutierten Ernährungsstrategien zur Gewichtsreduktion über einen Zeitraum von 6 Monaten. Eine Gruppe von 53 Teilnehmerinnen wurde einer kohlenhydratarmen Diät (LC), 54 Teilnehmerinnen einer fettarmen Diät (LF) und 53 Teilnehmerinnen einer fettarmen Diät mit gleichzeitiger Beschränkung von Kohlenhydraten mit hoher glykämischer Last auf maximal $50 \mathrm{~g} / \mathrm{Tag}$ (LFRGL) zugeordnet. Die diätetischen Vorgaben beschränkten in den ersten 4 Wochen die Kohlenhydratmenge auf maximal $30 \mathrm{~g}$ und Fettmenge auf $30 \mathrm{~g} / \mathrm{Tag}$. Für die folgenden 5 Monate wurden $60 \mathrm{~g}$ Kohlenhydrate und $45 \mathrm{~g}$ Fett/Tag erlaubt. Alle Teilnehmerinnen erhielten die Informationen zu ihren diätetischen Vorgaben in rein schriftlicher Form. Vor Beginn, nach 4 Wochen und nach 6 Monaten der Ernährungsintervention wurden Blutuntersuchungen sowie Messungen von Blutdruck und Bauchumfang vorgenommen. Das Körpergewicht der Probandinnen wurde während des gesamten Verlaufs wöchentlich kontrolliert.

Nach 4 Wochen beziehungsweise 6 Monaten hatten die Teilnehmerinnen durchschnittlich in LC -4,4 kg beziehungsweise -8,1 kg abgenommen, die Frauen in LF -3,3 kg beziehungsweise -6,3 kg und die in LFRGL -3,6 kg beziehungsweise -6,8 kg. Die Unterschiede zwischen den Gruppen waren mit p=0,4868 zu keinem Zeitpunkt signifikant. Die Abbrecherquote dagegen liegt mit 45,3\% in der Gruppe LC deutlich höher als in LF mit 35,2\% oder LFRGL mit 30,2\%.

Die Verlaufsbeobachtung verschiedener kardiovaskulärer Risikoparameter unter den Diätinterventionen zeigt nach 4 Wochen einen signifikanten Anstieg des Homocysteins in der Gruppe LC ( $p=0,0144)$ gegenüber unwesentlichen Veränderungen in LF $(p=0,6896)$ und LFRGL ( $\mathrm{p}=0$,5255). Eine statistisch signifikante Verminderung des hsCRP bleibt ebenfalls nur in der kohlenhydratarmen Gruppe trotz tendenziell größter Gewichtsabnahme aus ( $\mathrm{p}=0,6182$ ), während hsCRP über den gesamten Beobachtungszeitraum in LF ( $p=0,0027)$ und LFRGL (0,0292) absinkt. Eine direkte Korrelation zwischen diesen Parametern konnte nicht nachgewiesen werden. Für beide Ergebnisse kann dennoch ein gemeinsamer Mechanismus als Ursache angenommen werden. Denkbar wäre ein Minderverzehr und Mangel an bestimmten Vitaminen. Der Minderverzehr fällt besonders für das kritische Vitamin Folsäure, aber auch für Vitamin B6 in der Gruppe LC signifikant aus. 
Der verringerte Verzehr verschiedener Vitamine und Ballaststoffe unter den diätetischen Regimen erscheint gesundheitlich insgesamt nicht unbedenklich, wobei nur die kohlenhydratarme Diät eine klinisch relevante Einschränkung des Vitaminverzehrs und einen Anstieg von Homocystein und fehlenden Abfall des hsCRP hervorrief. Bei niedrigem Ausgangsverzehr der Folsäure unterhalb der von der DGE empfohlenen Zufuhrmenge von $400 \mu$ /Tag sollte die generelle Empfehlung einer Folsäuresupplementation unter Ernährungsstrategien zur Gewichtsreduktion und besonders unter kohlenhydratarmer Ernährung angeregt werden.

Die Veränderungen der anderen kardiovaskulären Risikofaktoren wie Triglyceride, Gesamtcholesterin, HDL, LDL, Glucose, systolischer und diastolischer Blutdruck entsprechen weitgehend den Ergebnissen aus der Literatur und zeigen keine signifikanten Differenzen zwischen den Gruppen.

Zusammenfassung der wichtigsten Ergebnisse

\begin{tabular}{|c|c|c|c|}
\hline Differenz der Mittelwerte & Gruppe LC & Gruppe LF & Gruppe LFRGL \\
\hline Gewicht T2-T0 (kg) & $-8,1$ & $-6,3$ & $-6,8$ \\
\hline Homocystein T1-T0 ( $\mu \mathrm{mol} / \mathrm{l})$ & 3,6 * & 0,7 & 0,5 \\
\hline HsCRP T2-T0 (mg/l) & $-0,04$ & $-0,87^{*}$ & $-0,39 *$ \\
\hline TriglycerideT2-T0 (mg/dl) & $-42,7^{*}$ & $-20,2$ & 0,4 \\
\hline Ballaststoffe T2-T0 (g/Tag) & $-13,8^{*}$ & 2,7 & $3,8^{*}$ \\
\hline Vitamin B6 (mg/Tag) & $-0,5^{*}$ & $-0,1$ & $-0,3 *$ \\
\hline Folsäure T2-T0 ( $\mu \mathrm{g} / \mathrm{Tag})$ & $-56,2$ * & $-19,1$ & 6,3 \\
\hline
\end{tabular}

* Statistisch signifikante Differenzen zwischen den Zeitpunkten

Tabelle 41: Übersicht über die wichtigsten Ergebnisse 


\section{Literaturliste}

$1 \quad$ Adam O (2003):

Rheumatische Erkrankungen; in: Ernährungsmedizin - Prävention und Therapie; 2. Auflage; hrsg. v. Schauder P, Ollenschläger G; Urban \& Fischer Verlag, München und Jena 2003, 863-874

2 Anderson JW, Konz EC (2001):

Obesity and disease management: Effect of weight loss on comorbid conditions. Obes Res $\underline{9}$, 326-334

3 Atkins RC:

Dr. Atkins' new diet revolution. Simon \& Schuster, New York 1998

4 Atkins RC und Herwood RW:

Dr. Atkins Diät-Revolution. Gouverts Krüger Stahlberg Verlag GmbH, Frankfurt/M. 1974

5 Bonaa KH, Njolstad I, Ueland PM, Schirmer H, Tverdal A, Steigen T, Wang H, Nordrehaug JE, Arnesen E, Rasmussen K (2006):

Homocysteine lowering and cardiovascular events after acute myocardial infarction. N Engl J Med $\underline{354}, 1578-1588$

6 Brattström L, Wilcken DEL (2000):

Homocysteine and cardiovascular disease: cause or effect? Am J Clin Nutr $\underline{72}$, 315-323

7 Bravata DeM, Sanders L, Huang J, Krumholz HM, Olkin I, Gardner CD, Bravata DaM (2003):

Efficacy and savety of low-carbohydrate diets. JAMA $\underline{289}, 1837-1850$

8 Bray GA, Popkin BM (1998):

Dietary fat intake does affect obesity! Am J Clin Nutr $\underline{68}, 1157-1173$

9 Dansinger ML, Gleason JA, Griffith JL, Selker HP, Schaefer EJ (2005):

Comparison of the Atkins, Ornish, Weight Watchers, and Zone diet for weight loss and heart disease reduction. JAMA $\underline{293}, 43-53$ 
10 DGE (Deutsche Gesellschaft für Ernährung):

Ernährungsbericht 1996. Druckerei Heinrich, Frankfurt/M. 1996

11 DGE (Deutsche Gesellschaft für Ernährung):

Ernährungsbericht 2000. Druckerei Heinrich, Frankfurt/M. 2000

12 DGE (Deutsche Gesellschaft für Ernährung):

DGE-Beratungsstandards. Deutsche Gesellschaft für Ernährung, Frankfurt/M. 2001

13 DGE (Deutsche Gesellschaft für Ernährung), ÖGE (Österreichische Gesellschaft für Ernährung), SGE (Schweizerische Gesellschaft für Ernährung), SVE (Schweizerische Vereinigung für Ernährung):

Referenzwerte für die Nährstoffzufuhr. Umschau/Braus, Frankfurt/M. 2000, 69-144

14 Dixon JB, Dixon ME, O’Brien PE (2001):

Elevated homocysteine levels with weight loss after lap-band surgery: higher folate and vitamin B12 levels required to maintain homocysteine level. Int J Obes Relat Metab Disord 25: 219-227

15 Ellrott T:

Der Einfluss der ad-libitum-Substitution normal fetthaltiger Lebensmittel durch fettreduzierte Leichtprodukte auf Körpergewicht, Serum-Blutparameter, Blutdruck und Lebensqualität. Med. Diss. Göttingen 1994

16 Ellrott T, Pudel V (2005):

Kohlenhydratarme Diäten (Low-Carb) zur Gewichtsreduktion. Ernährungsumschau $\underline{52}, 48-51$

17 Ellrott T, Beilschmidt S, Spirik J, Liechtenstein S, Neuhäuser-Berthold M, Pudel V (1998):

Der 1:1-Austausch normaler Lebensmittel durch vergleichbare fettärmere Lebensmittel bei Kohlenhydratverzehr ad libitum. Ernährungsumschau 45, 44-49 
18 Esposito K, Pontillo A, Di Palo C, Giuligliano G, Masella M, Marfella R, Giugliano D (2003):

Effects of weight loss and lifestyle changes on vascular inflammatory markers in obese women. JAMA $\underline{289}, 1799-1804$

19 Esposito K, Marfella R, Ciotola M, Di Palo C, Giugliano F, Giugliano G, D’Armiento M, D’Andrea F, Giugliano D (2004):

Effect of a mediterranean-style diet on endothelial dysfunction and markers of vascular inflammation in the metabolic syndrome. JAMA $\underline{292}, 1440-1446$

20 Extra Tip (2005):

In kleinen Schritten abnehmen. Prof. Pudel: Uni startet neues Therapieprogramm. Aus: Extra Tip, hrsg. Extra Tip Werbungs- und Vertriebsgesellschaft mbH, Göttingen, Ausgabe vom 16.01.2005

21 Faustin V:

Evaluierung und Weiterentwicklung der computergestützten Verzehrserhebungsmethode MediTouch. Oecotroph. Diss. Gießen 2004

22 Ford ES (1999):

Body mass index, diabetes, and C-reactive protein among U.S. adults. Diabetes Care 22, 1971-1977

23 Foster GD, Wyatt HR, Hill JO, McGuckin BG, Brill C, Mohammed S, Szapary PO, Rader DJ, Edman JS, Klein S (2003):

A randomized trial of low-carbohydrate diet for obesity. N Engl J Med 348, 2082-2090

24 Freedman MR, King J, Kennedy E (2001):

Popular diets: A scientific review. Obes Res $\underline{9}$, Suppl. 1, 1-40

25 Friso S, Jacques PF, Wilson PWF, Rosenberg IH, Selhub J (2001):

Low circulating vitamin B6 is associated with elevation of the inflammation marker C-reactive proteine independently of plasma homocysteine levels. Circulation $\underline{103}, 2788-2791$ 
26 Graham IM, Daly LE, Refsum H (1997):

Plasma homocysteine as a risk factor for vascular disease. The European concerted action project. JAMA $\underline{277}, 1775-1781$

27 Groeneveld M (2004):

Brauchen wir eine neue Ernährungspyramide? Ernährungsumschau 51, 308312

28 Guerre-Millo M (2004):

Adipose tissue and adipokines: for better or worse. Diabetes Metab 쓰, 13-19

29 Hahn A:

Vitamine; in: Ernährungsmedizin - Prävention und Therapie, 2. Auflage; hrsg.

v. Schauder P und Ollenschläger G; Urban \& Fischer Verlag, München, Jena 2003, 85-106

30 Hanson GK (2005):

Inflammation, atherosclerosis and coronary heart disease. N Engl J Med 352, 1685-1695

31 Hauner H, Buchholz G, Hamann A, Husemann B, Koletzko B, Liebermeister H, Wabitsch M, Westenhöfer J, Wirth A, Wolfram G:

Prävention und Therapie der Adipositas. Evidenzbasierte Leitlinie, Version 2005; Entwurf - Stand 4.10.05. Hrsg. von Deutsche Adipositas-Gesellschaft, Deutsche Diabetes Gesellschaft, Deutsche Gesellschaft für Ernährung, Deutsche Gesellschaft für Ernährungsmedizin, (www.adipositasgesellschaft.de/daten/Adipositas-Leitlinie-2005.pdf)

32 Holmquist C, Larsson S, Wolk A, De Faire U (2003):

Multivitamin Supplements Are Inversely Associated with Risk of Myocardial Infarction in Men and Women-Stockholm Heart Epidemiology Program (SHEEP). J Nutr 133, 2650-2654 


\section{Jarzemski S:}

Veränderung der Lebensmittelauswahl und Nährstoffversorgung durch Diäten:

"Low-Carb, Low-Fat und Low-Fat Reduced - Glycemic - Load“. Diplomarbeit des Fachbereichs Oecotrophologie der Hochschule Niederrhein, Abteilung Mönchengladbach, vorgelegt im Dezember 2005

34 Keil U:

Ernährungsepidemiologie; in: Ernährungsmedizin - Prävention und Therapie, 2. Auflage; hrsg. v. Schauder P und Ollenschläger G; Urban \& Fischer Verlag, München, Jena 2003, 14-25

35 King DE, Egan BM, Woolson RF, Mainous AG, Al-Solaiman Y, Jesri A (2007): Effect of a high-fiber diet vs a fiber-supplemented diet on C-reactive protein level. Arch Intern Med 167, 502-506

36 Kjeldsen-Kragh J, Haugen M, Borchgrevink CF, Laeruöm E, Eek M, Mowinkel P, Hovi K, Forre O (1991):

Controlled trial of fasting and one year on a vegetarian diet in rheumatoid arthritis. Lancet $\underline{338}, 899-902$

37 Liu S, Willett WC (2002):

Dietary glycemic load and atherothrombotic risk. Curr Atheroscl Rep $\underline{4}, 454-$ 461

38 Liu S, Willett WC, Stampfer MJ, Hu FB, Franz M, Sampson L, Hennekens CH, Manson JAE (2000):

A prospective study of dietary glycemic load, carbohydrate intake, and risk of coronary heart disease in US women. Am J Clin Nutr $\underline{71}$, 1455-1461

39 Lonn E, Yusuf S, Arnold MJO, Sheridan P, McQueen MJ, Pogue J, Probstfield J, Fodor G, Held C, Micks M et al.(The Heart Outcomes Prevention Evaluation (HOPE) 2 Investigators) (2006):

Homocysteine lowering with folic acid and B vitamins in vascular disease.

N Engl J Med $\underline{354}, 1567-77$

40 Ludwig DS (2000):

Dietary glycemic index and obesity. J Nutr $\underline{130}, 280-283$ 
41 Ludwig DS (2003):

Dietary glycemic index and the regulation of body weight. Lipids $\underline{38}, 117-121$

42 Macy EM, Hayes TE, Tracy RP (1997):

Variability in measurement of C-reactive protein in healthy subjects: implications for reference intervals and epidemiological application. Clin Chem $\underline{43}, 52-59$

43 Marcucci R, Zanazzi M, Bertoni E, Rosati A, Fedi S, Lenti M, Prisco D, Castellani S, Abbate R, Salvadori M (2003):

Vitamin supplementation reduces the progression of atherosclerosis in hyperhomocysteinemic renal-transplant recipients. Transplantation $\underline{75}, 1551$ 1555

44 McCully KS (1996):

Homocysteine and vascular disease. Nat Med 2, 386-389

45 Meckling KA, O'Sullivan C, Saari D (2004):

Comparison of a low-fat diet to a low-carbohydrate diet on weight loss, body composition, and risk factors for diabetes and cardiovascular disease in freeliving, overweight men and women. J Clin Endocrinol Metab 89, 2717-2723

46 Medina MA, Urdiales JL, Amores-Sanchez MI (2001):

Roles of homocysteine in cell metabolism - Old and new functions. Eur J Biochem 268, 3871-3882

47 Nader R, Ridker PM (2001 a):

High-sensitivity C-reactive protein: A novel promising marker of coronary heart disease. Clin Chem $\underline{47}$, 403-411

48 Nader R, Ridker PM (2001 b):

Proposed cardiovascular risk assessment algorithm using high-sensitivity Creactive protein and lipid screening. Clin Chem $\underline{47}, 28-30$

49 Nestlé Deutschland AG:

Kalorien mundgerecht. 12. Auflage; hrsg. v. Nestlé Deutschland AG, Umschau Buchverlag Breidenstein GmbH, Frankfurt/M. 2003 
50 Nurk E, Tell GS, Nygård O, Refsum H, Nilsen RM, Ueland PM (2004):

Changes in lifestyle and plasma total homocysteine: the Hordaland Homocysteine Study. Am J Clin Nutr $\underline{79}$, 812-819

51 Refsum H, Smith AD, Ueland PM, Nexo E, Clarke R, McPartlin J, Johnston C, Engbaek F, Schneede J, McPartlin C et al. (2004):

Facts and recommendations about total homocysteine determinations: An expert opinion. Clin Chem $\underline{50}$, 3-32

52 Rutter MK, Meigs JB, Sullivan LM, D’ Agostino RB, Wilson PWF (2004): C-reactive protein, the metabolic syndrome, and prediction of cardiovascular events in the Framingham Offspring Study. Circulation 110, 380-385

53 Samaha FF, Iqbal N, Seshadri P, Chicano KL, Daily DA, McGrory J, Williams T, Williams M, Gracely EJ, Stern L (2003):

A low-carbohydrate as compared with a low-fat diet in severe obesity. N Engl J Med 348, 2074-2081

54 Schnyder G, Roffi M, Pin R, Flammer Y, Lange H, Eberli FR, Meier B, Turi ZG, Hess OM (2001):

Decreased rate of coronary restenosis after lowering of plasma homocysteine levels. N Engl J Med $\underline{345}, 1593-1600$

55 Sheu WH, Wu HS, Wang CW, Wan CJ, Lee WJ (2001):

Elevated homocysteine concentrations six month after gastroplasty in morbidly obese subjects. Intern Med $\underline{40}, 584-588$

56 Sloth B, Krog-Mikkelsen I, Tetens I, Björck I, Vinoy S, Elmståhl H, Astrup A, Lang V, Raben A (2004):

No difference in body weight decrease between a low-glycemic-index and a high-glycemic-index diet but reduced LDL cholesterol after 10-wk ad libitum intake of low-glycemic-index diet. Am J Clin Nutr $\underline{80}$, 337-347 
57 Stern L, Iqbal N, Seshadri P, Chicano KL, Daily DA, McGrory J, Williams M, Gracely EJ, Samaha FF (2004):

The effect of low-carbohydrate versus conventional weight loss diets in severly obese adults: One year of follow-up of a randomized trial. Ann Intern Med $\underline{140}, 778-785$

58 Tracy RP (2001):

Is visceral adiposity the "enemy within"? Arterioscler Thromb Vasc Biol 21, 881-883

59 Ueland PM, Refsum H, Beresford SAA, Vollset SE (2000 a):

The controversy over homocysteine and cardiovascular risk. Am J Clin Nutr $\underline{72}, 324-332$

60 Ueland PM, Refsum H, Schneede J:

Determinants of plasma homocysteine; in: Homocysteine and vascular disease; hrsg. v. Robinson K; Kluwer Academic Publishers, Dordrecht/ Niederlande 2000 b, 59-84

61 Verhaar MC, Stroes E, Rabelink TJ (2002):

Folates and Cardiovascular Disease. Arterioscler Thromb Vasc Biol 22: 6-13

62 Verhoef P, Katan MB (2004):

A healthy lifestyle lowers homocysteine, but should we care? Am J Clin Nutr

$\underline{79}, 713-714$

63 Von Ahsen N, Schütz E, Armstrong VW und Oellerich M (1999):

Rapid detection of prothrombotic mutations of prothrombin (G20210A), factor V (G1691A), and methylenetetrahydrofolate reductase (C677T) by real-time fluorescence PCR with the LightCycler. Clin Chem $\underline{45}$, 694-696

64 WHO:

Obesity-preventing and managing the global epidemic. Report of a WHO

Consultation on Obesity. Technical Report Series 894, WHO, Geneva 2000

65 WHO:

The world health report 2001. Mental health: new understanding, new hope.

WHO, Geneva 2001 
66 WHO Europa:

Faktenblatt EURO/13/05. WHO, Kopenhagen/Bukarest 2005

67 Yudkin JS, Stehouwer CDA, Emeis JJ, Coppack SW (1999):

C-reactive protein healthy subjects: Associations with obesity, insulin resistance, and endothelial dysfunction. Arterioscler Thromb Vasc Biol $\underline{19}$, 972-978 


\section{Lebenslauf}

In Vechta, Niedersachsen, wurde ich am 08. März 1972 geboren. Dort verbrachte ich meine gesamte Schulzeit bis zum Abitur 1991 am Gymnasium „Unserer lieben Frau“. Anschließend studierte ich von Oktober 1991 bis März 1993 an der Westfälischen WilhelmsUniversität zu Münster drei Semester Chemie/Diplom ohne Abschluss. Im April 1993 wechselte ich zum Studium der Humanmedizin an die Georg-August-Universität nach Göttingen. Während des Medizinstudiums legte ich von April bis September 1996 ein FreiSemester für einen Auslandsaufenthalt in Mexiko zum Erwerb von spanischen Sprachkenntnissen und für Famulaturen ein. Nach dem Physikum im März 1995, dem 1. Staatsexamen im März 1996 und dem 2. Staatsexamen im August 1998 beendete ich mein Medizinstudium im Oktober 1999 mit dem 3. Staatsexamen am Albert-Schweitzer-Krankenhaus in Northeim. Die Tertiale Chirurgie und Anästhesie als Wahlfach hatte ich in Northeim absolviert. Das erste Tertial Innere Medizin absolvierte ich von Oktober 1998 bis Februar 1999 als ErasmusStudentin am Universitäts-Krankenhaus „12 de Octubre“ der Universidad Complutense in Madrid, Spanien.

Meine Vollapprobation als Ärztin erhielt ich nach meiner Zeit als ÄiP von November 1999 bis Mai 2001 in der Belegabteilung der kardiologischen Gemeinschaftspraxis PD Dr. Nordbeck, Prof. Dr. Wolpers und Dr. Becker in Göttingen. Insgesamt arbeitete ich dort bis Februar 2002, seit Mai 2001 als Assistenzärztin. Von März 2002 bis Dezember 2005 war ich als Assistenzärztin im Albert-Schweitzer-Krankenhaus in Northeim in der Inneren Medizin tätig. Seit März 2006 bin ich Assistenzärztin in der Diabetesklinik im Herz- und Diabeteszentrum NRW in Bad Oeynhausen.

Nebenberuflich erwarb ich die Zusatzqualifikationen Rettungsmedizin im März 2003 und Ernährungsmedizin im September 2005. 